\title{
EOL AND ETOL FORMS
}

This chapter is devoted to the study of EOL and ETOL forms, that is $L$ forms. The major distinction between the study of $L$ forms and grammar forms is that the natural mode of interpretation for $L$ forms is the s-interpretation. Indeed as we shall see, applying $g$-interpretations to $L$ forms leads to a loss of similarity rather than its retention. This change of emphasis together with the influence of parallel, as against sequential, rewriting leads to substantially different results.

For example, after introducing EOL and ETOL forms in section III.], we study reduction theorems and normal form theorems in section III.2. Although many reduction results are obtained it is shown that an EOL form cannot always be reduced to a form equivalent one which is propagating ( $\lambda$-free) or to one which is synchronized.

In the remaining sections a number of topics are discussed which were first introduced for L forms. In Section III.3 completeness is discussed, that is when does an EOL form (ETOL form) generate $\mathcal{L}$ (EOL) ( $\mathscr{L}$ (ETOL). We will demonstrate that even for two-symbol forms the picture is far from complete. In section III.4 the notions of goodness and very-completeness are discussed. In Section III.5 not only is it proved that the relations $\underset{s}{\Delta}$ and $\underset{g}{a}$ are decidable for both grammar and $L$ forms, but they are also shown to be NP-complete problems. Some other results on decidability of form equivalence are given also. Finally in Section III.6 the generative capacity of EOL forms is discussed with respect to context-free grammatical families. The question of which context-free grammatical families are EOL form families, is partially answered. Also an EOL form version of a generator is considered as well as the notions of regular-sufficiency and boundedness. 
III. 1 The Basics of EOL and ETOL Forms

Recall from Section I.1.2 that an EOL grammar is a couple ( $G, \Rightarrow$ ), where $G$ is a production scheme $(V, \Sigma, P, S)$ is which $V$ is an alphabet, $\Sigma \subseteq V, P \subseteq V \times V^{*}$, with at least one production in $P$ for each symbol in $V$ and $S$ is in $V-\Sigma$. Similarly an n-tabledETOL grammar is a couple $(G, \Rightarrow)$, where $G$ is a production scheme $\left(V, \Sigma, P_{1}, \ldots, P_{n}, S\right)$ in which $P_{i} \subseteq V \times V^{*}, I \leq i \leq n$ and with at least one production in each $P_{i}$ for each symbol in $V$. Each $P_{i}$ is referred to as a table. When $n=1$ we obtain an EOL grammar as a special case. Following Section $1.1 .2 \Rightarrow$ is a relation over $V *$ induced by the $P_{i}, 1 \leq i \leq n$, and as usual we obtain $\Rightarrow^{+}, \Rightarrow^{*}$ and $\Rightarrow^{i}, i \geq 0$. Notice that terminal rewriting is allowed by the definition of $\Rightarrow$ and $P_{i}$.

Letting $M, N \subseteq V *$ then the notation $M \rightarrow N$ denotes $\{\alpha \rightarrow \beta: \alpha$ in $M$ and $\beta$ in $N$ \}. We now turn to the central definitions of this chapter, firstly, for EOL forms and secondly for ETOL forms.

\section{Definition}

Let $G_{i}=\left(V_{i}, \Sigma_{i}, P_{i}, S_{i}\right), i=1,2$ be two EOL grammars. We say $\underline{G}_{2}$ is an $(s)$-interpretation of $G_{7}$ modulo $\mu$, denoted $G_{2} \underset{s}{\rightarrow} G_{1}(\mu)$, where $\mu$ is a dfl-substitution on $V_{i}^{*}$ if conditions (i) through (iv) obtain:

(i) $\mu(A) \leq V_{2}-\Sigma_{2}$, for al1 A in $V_{1}-\Sigma_{1}$,

(ii) $\mu(a) \subseteq \Sigma_{2}$, for all a in $\Sigma_{1}$,

(iii) $P_{2} \subseteq \mu\left(P_{1}\right)$, where $\mu\left(P_{1}\right)=\bigcup_{X \rightarrow \alpha \text { in } P_{1}} \mu(X) \rightarrow \mu(\alpha)$, and

(iv) $s_{2}$ is in $\mu\left(s_{1}\right)$.

We do not use g-interpretations for the following reasons.

First $\mu\left(P_{1}\right)$ may contain productions of the type:

$\lambda \rightarrow \beta$ or $a b c \rightarrow \beta$

as images of a production a $\rightarrow \alpha$, where a is terminal. Both $\lambda \rightarrow \beta$ and $a b c \rightarrow \beta$ are neither EOL nor ETOL productions, since such productions must have a single symbol on the left hand side.

Second two productions

$a \rightarrow a b$ and $b \rightarrow a$

could under g-interpretations give rise to

$a \rightarrow a b b$ and $b \rightarrow a a$

which means that the derivation tree: 


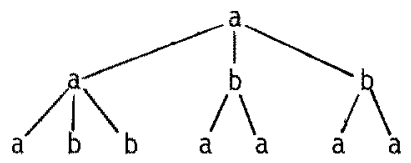

is an "image" of the

derivation tree:

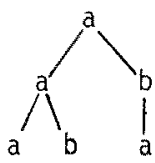

Third two productions

$a \rightarrow a b$ and $b \rightarrow a$

could under g-interpretations give rise to the productions

$c \rightarrow c c$ and $c \rightarrow c$,

that is a and b are "merged". In this case the derivation tree:

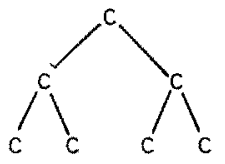

can be obtained, which must be the

"image" of:

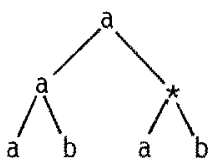

where * must be both $a$ and $b$. In other words no such tree exists in the EOL grammar defined by $a \rightarrow a b$ and $b \rightarrow a$.

However under s-interpretations none of the above problems can occur. Moreover our choice is mathematically more convenient since for each dfl-substitution $\mu$, we can define in a natural manner a $\mu^{-1}$ which satisfies $\mu^{-1} \mu(X)=X$ if $\mu(X) \neq \emptyset$.

When $P_{2}=\mu\left(P_{1}\right)$ we say that $G_{2}$ is a full s-interpretation of $G_{1}$ modulo $\mu$, denoted $G_{2} \triangleleft G_{1}$. Similarly we often drop the prefix sif no confusion results. An EOL form is simply an EOL grammar.

The collection of s-interpretation EOL forms obtained from a given EOL form $G$ is denoted by $s(G)$ and is called the $s$-EOL grammar family of $G$. Similarly the collection of languages obtained from a given EOL form $G$, denoted $\mathcal{L}_{S}(G, \Rightarrow)$ is defined by:

$\mathcal{L}_{S}(G, \Rightarrow)=\left\{L\left(G^{\prime}, \Rightarrow\right): G^{\prime}{ }_{S} G\right\}$ and is called the s-EOL grammatical family of $G$ or simply the language family of $G$. We say two EOL forms $G_{1}$ and $G_{2}$ are s-form equivalent if $\mathcal{Z}_{s}\left(G_{1}, \Rightarrow=\mathcal{Z}_{s}\left(G_{2}, \Rightarrow\right)\right.$, and strong s-form equivalent if $\xi_{s}\left(G_{1}\right)=\xi_{s}\left(G_{2}\right)$.

As for grammar forms, given two $E O L$ forms $G_{1}$ and $G_{2}$ it is decidable whether $G_{1} \Delta G_{2}$ and hence whether $\xi_{s}\left(G_{1}\right)=\xi_{s}\left(G_{2}\right)$, see Section 5.1. As for grammar forms, $\xi_{s}\left(G_{1}\right) \stackrel{g}{=} \xi_{s}\left(G_{2}\right)$ iff $G_{1} \underset{s}{a} G_{2}$ and $G_{2} \underset{s}{\Delta} G_{1}$ since $\underset{s}{\Delta}$ is reflexive and transitive.

Before considering other carry over results we look at some examples.

Let $F_{1}$ be defined by the productions:

$S \rightarrow a ; S \rightarrow S a ; a \rightarrow a$

then $L\left(F_{1} \Rightarrow\right)=\left\{a^{i}: i \geq 1\right\}$. Since $a \rightarrow a$ in $F_{1}$ is the only production 
for the only terminal symbol and it is the "identity" production, we say $F$ is terminally stable. Any EOL grammar which is terminally stable generates a context-free language. In this case since $F$ is left linear as far as the nonterminal productions are concerned, then each interpretation $F^{\prime}$ a $F$ will also be left linear in this sense. Hence if $F^{\prime}$ is terminally stable it is straightforward to see that $L\left(F^{\prime} \Rightarrow\right)$ is in $\mathcal{L}(\mathrm{REG})$. Therefore

$$
\mathcal{L}(R E G) \subseteq \mathcal{L}_{\mathrm{s}}\left(F_{1}, \Rightarrow\right.
$$

It will be shown that $\mathcal{L}_{S}\left(F_{1} \Rightarrow\right)=\mathcal{L}(R E G)$ and $F_{1}$ is, therefore, regular complete, see Section 3.4 .

Secondly, let $F_{2}$ be defined by:

$S \rightarrow a ; S \rightarrow S ; S \rightarrow S S ; a \rightarrow S$

then again $L\left(F_{2}, \Rightarrow\right)=\left\{a^{i}: i \geq 1\right\}$. However consider an arbitrary $E O L$ language $L$. It is well known that $L$ can be generated by an EOL grammar $G$ which only has productions of types:

(i) $A \rightarrow a$, (ii) $A \rightarrow B$, (iii) $A \rightarrow B C$, (iv) $a \rightarrow N$ and clearly such a $G$ has the property that

$$
G \underset{s}{4} F_{2} \text {. }
$$

This immediately implies that $\mathcal{L}_{\mathrm{s}}\left(\mathrm{F}_{2}, \Rightarrow\right)=\mathcal{L}(\mathrm{EOL})$, that is, $\mathrm{F}_{2}$ is EOL-complete.

These two examples initiate the studies of EOL-completeness, regular-completeness and the comparison of grammatical families and EOL-grammatical families. We return to these topics in later sections. Recall that under g-interpretations, the question of whether or not $\mathcal{L}_{g}(G, \Rightarrow)=\mathcal{L}(C F)$, that is, context-free-completeness, is trivial. That this is not so for EOL-completeness is seen by considering $F_{3}$ and $\mathrm{F}_{4}$ which will be shown to be EOL-complete in Section $3.1 . \mathrm{F}_{3}$ is defined by:

$$
S \rightarrow a ; S \rightarrow S ; S \rightarrow a S ; a \rightarrow S
$$

and $F_{4}$ is defined by:

$$
S \rightarrow a ; S \rightarrow a S ; S \rightarrow S a ; a \rightarrow a ; a \rightarrow S ; a \rightarrow S S \text {. }
$$

Notice that in both $\mathrm{F}_{3}$ and $\mathrm{F}_{4}$ expansion occurs via terminal rewriting and in $F_{4}$ looping also occurs via terminal rewriting. The proof that $\mathrm{F}_{4}$ is EOL-complete necessitates using a novel normal form theorem for EOL grammars.

We now define interpretations of ETOL forms in a similar manner to that for EOL forms.

\section{Definition}

$$
\text { Let } G_{i}=\left(V_{j}, \Sigma_{i}, P_{i, 1}, \ldots, P_{i, n}, S_{i}\right), i=1,2 \text { be two } n \text {-tabled }
$$

ETOL forms (or grammars). We say $\underline{G}_{2}$ is an $s$-interpretation of $G_{1}$ 
modulo $\mu$, denoted $G_{2} \underset{s}{\Delta} G_{1}(\mu)$, where $\mu$ is a dfl-substitution on $V_{1}^{*}$, if conditions ( $i$ ) through (iv) hold:

(i) $\mu(A) \subseteq V_{2}-\Sigma_{2}$, for all $\mathrm{A}$ in $V_{1}-\Sigma_{1}$,

(ii) $\mu($ a $) \subseteq \Sigma_{2}$, for all a in $\Sigma_{1}$,

(iii) for all $i, 1 \leq i \leq n, P_{2, i} \leq \mu\left(P_{1, i}\right)$, and

(iv) $s_{2}$ is in $\mu\left(s_{1}\right)$.

If $P_{2, i}=\mu\left(P_{1, i}\right)$ for al1 $i, 1 \leq i \leq n$ then $G_{2}$ is a full

s-interpretation of $G_{1}$, denoted $G_{2} \overrightarrow{f s} G_{1}(\mu)$. As before we often write $G_{2} \underset{S}{\Delta} G_{1}$ rather than $G_{2} \underset{S}{a} G_{1}(\mu)$ and speak of interpretation, rather than s-interpretation.

It is important to observe that the number of tables is preserved under interpretation. This is, of course, not the only possible definition, but it seems the most natural. We could however have allowed the interpretation grammar to have an arbitrary number of tables or perhaps at most $n$ tables. Neither of these alternatives have been investigated and therefore we only consider the table preserving interpretations we have introduced.

As for $E O L$ forms we can define $\xi_{S}(G), \mathcal{Z}_{S}(G, \Rightarrow)$, s-form equivalence and strong $s-f o r m$ equivalence. Let us consider some examples.

Let $\mathrm{H}_{1}$ defined by:

$\{S \rightarrow a ; S \rightarrow S ; S \rightarrow S S ; a \rightarrow S\},\{S \rightarrow S ; a \rightarrow S\}$

be a two-tabled ETOL form. Now $\mathcal{L}_{S}\left(H_{1} \Rightarrow\right)=\mathcal{Z}$ (ETOL) since every ETOL language can be generated by a two-tabled ETOL grammar, in which the first table has productions of the types

(i) $A \rightarrow a,(i i) A \rightarrow B,(i i i) A \rightarrow B C$, and (iv) $a \rightarrow N$

and the second table has productions of the types

(v) $A \rightarrow B$, and (vi) a $\rightarrow \mathrm{N}$.

It is clear that such an ETOL grammar is an interpretation of $\mathrm{H}_{7}$.

Consider $\mathrm{H}_{2}$ defined by:

$\{S \rightarrow S ; S \rightarrow S S ; a \rightarrow a\},\{S \rightarrow a ; a \rightarrow a\}$.

Now $\mathrm{H}_{2}$ is terminally stable, and $L\left(H_{2}, \Rightarrow\right)=\left\{a^{i}: i \geq 1\right\}$, but in contrast to the situation with EOL forms consider $\mathrm{H}_{2} \underset{\mathrm{s}}{4} \mathrm{H}_{2}$ given by:

$$
\{S \rightarrow S S ; a \rightarrow a\},\{S \rightarrow a ; a \rightarrow a\} \text {, }
$$

again $H_{2}^{\prime}$ is terminally stable, but in this case $L\left(H_{2}^{\prime} \Rightarrow\right)$ is not in $\mathcal{L}(\mathrm{CF})$. In fact

$$
L\left(H_{2}, \Rightarrow\right)=\left\{a^{2}: i \geq 0\right\} \text {. }
$$

Thus in ETOL forms terminal stability does not necessarily imply context-freeness. This is because in $\mathrm{H}_{2}$ we can still "synchronize" 
via the second tabie, which always changes a nonterminal word into a terminal word.

Now letting $L$ be an arbitrary EOL language, it can be generated by a synchronized EOL grammar $G$ whose productions are only of types: (i) $A \rightarrow a$, (ii) $A \rightarrow B$, (iii) $A \rightarrow B C$ and (iv) $a \rightarrow N$. Since each terminal producing derivation only introduces terminals at the final derivation step, then we can split the productions of $G$ into two classes:

(a) types (ii), (iii)

and (b) type (i).

It should be clear that class (a) productions are interpretations of the first table of $\mathrm{H}_{2}$ and class (b) productions of the second table. Let $a \rightarrow a$ be in both tables for all terminals a in $G$, then we have obtained $L$ via an interpretation of $H_{2}$. Therefore $\not{Z}(E O L) \subseteq \mathscr{Z}_{\mathrm{S}}\left(\mathrm{H}_{2}, \Rightarrow\right)$. In fact, it will be shown that $\mathcal{Z}_{\mathrm{s}}\left(\mathrm{H}_{2}, \Rightarrow\right)=\mathcal{L}$ (EOL) in Section 3.3 .

Finally, consider $\mathrm{H}_{3}$, a three-tabled ETOL form defined by:

$\{S \rightarrow S ; S \rightarrow S S ; a \rightarrow a\},\{S \rightarrow S ; a \rightarrow a\},\{S \rightarrow a ; a \rightarrow a\}$

then by similar arguments to those for $\mathrm{H}_{1}$ and $\mathrm{H}_{2}$ it $\mathrm{can}$ be shown that $\mathcal{L}($ ETOL $) \subseteq \mathcal{L}_{\mathrm{s}}\left(\mathrm{H}_{3}, \Rightarrow\right.$ and hence $\mathrm{H}_{3}$ is ETOL-complete.

Before introducing two simulation lemmas, we first mention some results which carry over either directly from grammar forms, or in a similar way. We state them for ETOL forms only, since EOL forms are a special case.

\section{Theorem 1.1}

(i) The relation $\underset{s}{4}$ for ETOL forms is a preorder (cf. Section II.3) and is decidable (see Section 5 ).

(ii) Let $F_{1}$ and $F_{2}$ be ETOL forms, then $\xi_{s}\left(F_{1}\right) \subseteq \xi_{s}\left(F_{2}\right)$ iff $F_{1} \underset{s}{4} F_{2}$,

(iii) It is decidable for arbitrary ETOL forms $F_{1}$ and $F_{2}$ whether or not $\xi_{s}\left(F_{1}\right)=\xi_{s}\left(F_{2}\right)$ (see Section 5$)$,

(iv) For any two ETOL forms $F_{1}$ and $F_{2}$ an ETOL form $F$ can be constructed such that $\xi_{s}(F)=\xi_{s}\left(F_{1}\right) \cap \xi_{s}\left(F_{2}\right)$ (cf. Section II.3.3),

(v) For any ETOL form $F$ an essentialty unique ETOL form $F^{\prime}$ with a minimal number of productions in each table can be constructed such that $\xi_{s}(F)=\xi_{s}\left(F^{\prime}\right)$, (cf. Section II.3.4).

Note that the carry over of the results stated in this theorem are straightforward since they are only concerned with ETOL grammar families, not the language families.

Since the notion of an EOL morphism is unavailable we only have the following result, which corresponds to Theorem II.1.1. 


\section{Theorem 1.2}

Let $G_{i}=\left(V_{i}, \Sigma_{i}, P_{i, 1}, \ldots, P_{i, n}, S_{i}\right), i=1,2$ be two $n$-tabled ETOL forms such that $G_{2} \underset{S}{S} G_{T}(\mu)$ for some dfl-substitution $\mu$.

Then there is a letter-to-letter homomorphism $\mu^{-1}$ from $\mu\left(v_{1}\right)$ * to $V_{1}$ * satisfying the following conditions:

(i) For all $X$ in $V_{1}$, if $\mu(X) \neq \emptyset$, then for all $Y$ in $\mu(X), \mu^{-1}(Y)=X$, (ii) For all $X^{\prime} \rightarrow \alpha^{\prime}$ in $P_{2}, \mu^{-1}\left(X^{\prime}\right) \rightarrow \mu^{-1}\left(\alpha^{\prime}\right)$ is in $P_{1}$ and moreover $X^{\prime} \rightarrow \alpha^{\prime}$ is in $\mu\left(\mu^{-1}\left(X^{\prime}\right) \rightarrow \mu^{-1}\left(\alpha^{\prime}\right)\right)$.

In other words $\mu^{-1}$ is an inverse as far as the alphabet of $\mathrm{P}_{2}$ is concerned.

Since $\underset{S}{\$}$ is transitive we have immediately that for an arbitrary ETOL form $G, \mathcal{Z}_{\mathrm{S}}(G, \Rightarrow)$ is closed under dfl-substitution. As we shall see later this is the best possible result.

\section{III.1. Derivation Relationships}

The two techniques of isolation and simulation are as important for $L$ forms as for grammar forms. As in section II.2 we prepare the way for these techniques by remarking on the close relationship between derivations in the s-interpretation ETOL grammar and in its ETOL form grammar. Again we will often give results for ETOL forms since EOL forms are a special case of ETOL forms.

\section{Lemma 2.3}

Let $G_{i}=\left(V_{i}, \Sigma_{i}, P_{i}, 1, \ldots, P_{i, n}, S_{j}\right), i=1,2$ be two $n$-tabled ETOL forms such that $G_{2} \underset{s}{\Delta} G_{1}(\mu)$. Then for every derivation

$$
\alpha_{0}^{1} \Rightarrow \alpha_{1}^{1} \Rightarrow \ldots \alpha_{m}^{1} \text { in } G_{2} \text {, }
$$

for some $\alpha_{j}$ in $V_{2}^{*}, 0 \leq j \leq m$ and $m>0$, there is a derivation

$$
\alpha_{0} \Rightarrow \alpha_{1} \Rightarrow \ldots \alpha_{m} \text { in } G_{1} \text {, }
$$

for some $\alpha_{j}$ in $V_{l}^{*}$ such that $\alpha_{j}=\mu^{-1}\left(\alpha_{j}^{\prime}\right), 0 \leq j \leq m$.

\section{Proof: Clear.}

As in the case of grammar forms we obtain the following corollaries. 


\section{Corolzary 1.4}

Let $G_{i}, i=1,2$ be two $n$-tabled ETOL forms $s$ ch that $G_{2} \underset{s}{\Delta} G_{1}$. Then for every derivation tree in $G_{2}$ there is an equally shaped derivation tree in $G_{1}$.

\section{Corozzary 1.5}

Let $G_{i}, i=1,2$ be two $n$-tabled ETOL forms such that $G_{2} \underset{s}{\Delta} G_{1}$. Then, if $G_{2}$ is infinite then $G_{1}$ is infinite, but not conversely. This also implies that if $G_{1}$ is finite then $G_{2}$ is finite but not conversely.

Proof: This result is not quite as straightforward as is the case for grammar forms, since derivations in $G_{1}$ are of unbounded extent. However if $G_{1}$ is finite then only a finite number of word lengths are generated, even if they are generated infinitely often. Now by Lemma 1.3 no words can be generated by $G_{2}$ with lengths different from those of $G_{1}$. Hence $G_{2}$ is finite. Hence if $G_{2}$ is infinite $G_{1}$ must also be infinite.

\section{Corolzary 7.6}

Then

Let $G_{i}, i=1,2$ be two n-tabled ETOL forms such that $G_{2} \Delta_{s} G_{1}$.

(1) $G_{2}$ is looping implies $G_{1}$ is looping, and

(2) $G_{2}$ is expansive implies $G_{1}$ is expansive.

As for grammar forms we now obtain:

\section{Theorem 1.7}

Let $G$ be an arbitrary ETOL form. Then $\chi_{s}(G, \Rightarrow) \neq \mathcal{X}_{s}(F I N)$.

Proof: As for grammar forms, Theorem II.2.5.

\section{III.T.2 Isolation and Simulation}

Because derivations in EOL and ETOL forms are parallel in nature, the simulation results given below have to take into account the length of the simulating derivations. However, isolation is treated in exactly the same manner as for grammer forms, namely, mutually distinct derivations $A \Rightarrow{ }^{+} \alpha_{i}, 1 \leq i \leq n$, for some $n \geq 1$, are isolated by a suitable renaming of each symbol appearing in the derivations so that the new derivations $A \Rightarrow a_{j}$ are uniquely determined by the appearance of $A$. 
Recall that two derivations $A \Rightarrow^{+} \alpha$ and $A \Rightarrow^{+} B$ are distinct if neither is a prefix of the other. In fact the situation is simpler than in the grammar form case since at each intermediate step in $A \Rightarrow^{+} \alpha_{j}$, that is, for all $\gamma$ such that $A \Rightarrow^{+} \gamma \Rightarrow^{+} \alpha_{j}$, every symbol in $\gamma$ needs to be renamed.

For simplicity, consider the case of one derivation $A \Rightarrow^{+} \alpha$. This is easily generalized to the case $n>1$. Let $G=(V, \Sigma, P, S)$ be an EOL form and $A \Rightarrow+\alpha$ a derivation in $G$, defined by the following sequence:

$$
A=\alpha_{0} \Rightarrow \alpha_{1} \Rightarrow \ldots \Rightarrow \alpha_{m}=\alpha \text {, for some } m \geq 1 \text {. }
$$

In the case $m=1$ the derivation is trivially isolated, hence consider $m>1$. Let $\alpha_{i}=A_{i, 1} \ldots A_{i, n_{j}}, 0<i<m$, and define new symbols $[i, j]$, $1<j \leq n_{j}, 0<i<m$, which are terminal if $A_{i, j}$ is terminal and nonterminal otherwise. The derivation $(*)$ becomes:

$$
A=\alpha_{0} \Rightarrow[1,1] \ldots\left[1, n_{1}\right] \Rightarrow \ldots \Rightarrow[m-1,1] \ldots\left[m-1, n_{m-1}\right] \Rightarrow \alpha_{m}=\alpha
$$

where productions involving the new symbols are:

$$
\begin{aligned}
& \quad[i, j] \rightarrow[i+1, k] \ldots[i+1, \ell] \text { if } A_{i, j} \rightarrow A_{i+1, k} \ldots A_{i+1, l} \text { is in }(*), \\
& \text { for } 0<i<m-1 \text { and } \\
& {[m-1, j] \rightarrow A_{m, k} \ldots A_{m, \ell} \text { if } A_{m-1, j} \rightarrow A_{m, k} \ldots A_{m, l} \text { is in (*) }} \\
& \text { Hence we easily obtain the isolation lemma: }
\end{aligned}
$$

Lemma 1.8 - Isolation Lemma

Let $G=(V, \Sigma, P, S)$ be an EOL form, $A$ be in $V$ and let $A \Rightarrow^{+} \alpha_{i}$, $1 \leq i \leq n$ for some $n \geq 1$, be distinct derivations in $G$. Then there exists an EOL form $G^{\prime}=\left(V^{\prime}, \Sigma^{\prime}, P^{\prime}, S^{\prime}\right)$ such that

(i) $V \subseteq V^{\prime}, \Sigma \subseteq \Sigma^{\prime}, P-\{A \rightarrow \gamma: A \rightarrow \gamma$ in $P\} \subseteq P^{\prime}$ and $S^{\prime}=S$, (ii) G' $\rightarrow$ G and

(iii) whenever $A \Rightarrow^{+} \beta$ in $G^{\prime}$ with $\beta$ in $V *$ then $A \Rightarrow^{+} \alpha_{i} \Rightarrow^{*} \beta$, for some $i, 1 \leq i \leq n$, that is, either $\beta=\alpha_{i}$ or $\alpha_{i}$ occurs as an intermediate word in the derivation.

Although we make 1 ittle use of this result explicitly it is often used implicitly in the following. We say G' is an isolating interpretation of $G$.

We can obtain a similar isolation lemma for ETOL forms. However since we only consider isolation within a table in the following sections, this reduces to the EOL form case considered above, because all the other tables can be ignored.

We now turn to the simulation lemmas. These will be stated and proved for EOL forms, while the case of ETOL forms will be reduced to these. 
Let $G_{i}=\left(V_{i}, \Sigma_{i}, P_{i}, S\right), i=1,2$ be two EOL forms such that $V_{1}-\Sigma_{1} \subseteq V_{2}-\Sigma_{2}$ and $\Sigma_{1} \subseteq \Sigma_{2}$ and $\ell \geq 1$ be an integer such that for all $X \rightarrow \alpha$ in $P_{1}$ there is a derivation $X \Rightarrow \alpha$ in $G_{2}$. Then for each

$G_{1}^{\prime}=\left(V_{1}^{\prime}, \Sigma_{1}^{\prime}, P_{j}^{\prime}, S^{\prime}\right) \underset{S}{\Delta} G_{1}\left(\mu_{1}\right)$ an EOL grammar $G_{2}^{\prime}=\left(V_{2}^{\prime}, \Sigma_{2}^{\prime}, P_{2}^{\prime}, S^{\prime}\right) \underset{S}{\Delta} G_{2}\left(\mu_{2}\right)$ can be constructed such that:

$$
X^{\prime} \rightarrow \alpha^{\prime} \text { is in } P_{1}^{\prime} \text { iff } S^{\prime} \text { is in } \mu_{1}\left(V_{1}\right) \text { and } X^{\prime} \Rightarrow^{\prime} \alpha^{\prime} \text { in } G_{2}^{\prime} \text {. }
$$

Proof: For $\ell=1$ we have $P_{1} \subseteq P_{2}$ and hence $G_{2}^{\prime}$ can be chosen to be $G_{1}^{\prime}$. Therefore consider the case $\ell>1$. First define for all $X$ in $V_{2} \mu(X)=$ $\mu_{1}(X)$ for a $11 X$ in $V_{T}$ and $\mu(X)=\{X\}$, otherwise. Consider $G_{2}^{\prime \prime}=\left(V_{2}^{\prime \prime}, \Sigma_{2}^{\prime \prime}, P_{2}^{\prime \prime}, S^{\prime}\right) \underset{f S}{\Delta} G_{2}(\mu)$. Clearly $X^{\prime} \rightarrow \alpha^{\prime}$ is in $P_{1}^{\prime}$ implies $X^{\prime}$ is in $\mu\left(V_{1}\right)$ and $X^{\prime} \Rightarrow \alpha^{\prime}$ in $G_{2}^{\prime \prime} \quad$ Now use the isolation lemma repeatedly for each derivation $X^{\prime} \Rightarrow^{\prime} \alpha^{\prime}$ in $G_{2}^{\prime \prime}$ with $X^{\prime}$ in $\mu\left(V_{1}\right)$ to obtain $G_{2}^{\prime}$ fulfilling the conditions of the lemma.

Note that the conditions of Lemma 1.9 for $G_{1}$ and $G_{2}$ do not necessarily imply that $s\left(G_{1} \Rightarrow\right) \subseteq{ }_{s}\left(G_{2}, \Rightarrow\right)$. For example, let $G_{1}$ be $S \rightarrow a$; $a \rightarrow N ; N \rightarrow N$ and $G_{2}$ be $S \rightarrow b ; b \rightarrow a ; a \rightarrow M ; M \rightarrow N ; N \rightarrow M ;$ then $2=2$ and $X \rightarrow \alpha$ is in $P_{1}$ iff $X$ is in $V_{1}$ and $X \Rightarrow \alpha$ is in $G_{2}$.

However, $\mathcal{L}_{s}\left(G_{1} \Rightarrow \notin \mathcal{Z}_{s}\left(G_{2} \Rightarrow\right)\right.$ since each language in $\mathcal{L}_{s}\left(G_{2}, \Rightarrow\right.$ contains at least two words, while there are languages in $\alpha_{s}\left(G_{1}, \Rightarrow\right)$ which consist of exactly one word.

Notice that we have avoided a second difficulty, namely, nonterminals in $G_{1}$ may be terminals in $G_{2}$ or vice versa, by placing restrictions on the alphabets of $G_{1}$ and $G_{2}$.

\section{Lemma 1.10 - Expansion SimuZation Lemma}

Suppose $G_{i}=\left(V_{i}, \Sigma_{i}, P_{i}, S\right) i=1,2$ are two EOL forms such that $V_{1}-\Sigma_{1} \subseteq V_{2}-\Sigma_{2}, \Sigma_{1} \subseteq \Sigma_{2}$, and for some integer $\ell \geq 1$, $X \rightarrow \alpha$ in $P_{1}$ implies $X \underset{n t}{\Rightarrow} \stackrel{\text { im }}{\alpha}$ in $G_{2}$.

Then $\mathcal{L}_{s}\left(G_{1}, \Rightarrow\right) \subseteq \mathcal{L}_{s}\left(G_{2}, \Rightarrow\right)$.

Proof: Without loss of generality we may assume the stronger condition:

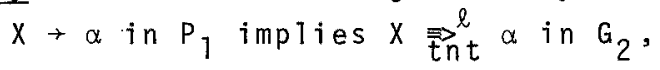

since we can always construct an isolating interpretation of $G_{2}$ so that this is true.

Consider $G_{1}^{\prime}, G_{2}^{\prime}, \mu_{1}$ and $\mu_{2}$ of Lemma 1.9. It is sufficient to prove $L\left(G_{j}^{\prime} \Rightarrow\right)=L\left(G_{2}^{\prime}, \Rightarrow\right)$, since this will imply $\mathcal{Z}_{s}\left(G_{1}, \Rightarrow \subseteq \mathcal{L}_{s}\left(G_{2}, \Rightarrow\right)\right.$. 
Now by Lemma 1.9 and the tnt-condition, $L\left(G_{1}, \Rightarrow\right) \subseteq L\left(G_{2}, \Rightarrow\right)$. To obtain the reverse inclusion consider an arbitrary word $x^{\prime}$ in $L\left(G_{2}^{\prime}, \Rightarrow\right)$.

Either $S^{\prime} \Rightarrow x^{\prime}$ in $G_{2}^{\prime}$, for some $m>0$ in which case by Lemma $1.9 S^{\prime} \Rightarrow x^{\prime}$ in $G_{j}^{\prime}$, or $S^{\prime} \Rightarrow^{m \ell^{2}} y^{\prime} \Rightarrow t x^{\prime}$ in $G_{2}^{\prime}$ with $1 \leq t \leq l-1$. In this latter case we show that $x^{\prime}$ is not in $L\left(G_{2}^{\prime}, \Rightarrow\right)$.

clearly, $S^{\prime} \Rightarrow y^{\ell} y^{\prime} x^{\prime} \Rightarrow^{l-t} z^{\prime}$ in $G_{2}^{\prime}$ for some $z^{\prime}$. Since $y^{\prime}$ is in $\mu_{7}\left(V_{1}\right)$ * and $y^{\prime} \Rightarrow^{\ell} z^{\prime}$ in $G_{2}^{\prime}$ we have:

$y^{\prime}=x_{1}^{1} \ldots x_{r}^{\prime} z^{\prime}=\alpha_{1}^{1} \ldots \alpha_{r}^{\prime}$ with $X_{i}^{\prime} \rightarrow \alpha_{i}^{\prime}$ in $P_{j}^{\prime}$, in other words $\mu_{i}^{-1}\left(x_{i}^{i}\right) \rightarrow \mu_{i}^{-1}\left(\alpha_{i}^{i}\right)$ is in $P_{1}, 1 \leq i \leq r$. Hence $u_{1}^{-1}\left(X_{i}^{\prime}\right) \operatorname{tnt}^{\ell} \mu_{1}^{-1}\left(\alpha_{i}^{\prime}\right)$ in $G_{2}$,

thus

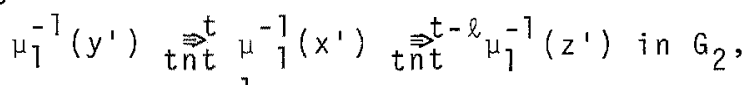

which implies $\mu_{1}^{-1}\left(x^{\prime}\right)$ contains a nonterminal and hence $x^{\prime}$ contains a nonterminal. Therefore $x^{\prime}$ is not in $L\left(G_{2}^{\prime}, \Rightarrow\right)$.

As we have seen previous to the lemma the nt-condition cannot be dropped.

We now turn to a converse simulation result in which derivations are "contracted" into productions.

\section{Lemma 1.11-- Contraction Simuzation Lemma}

Suppose $G_{i}=\left(V_{i}, \Sigma_{i}, P_{i}, S\right), i=1,2$ are two EOL forms such that $V_{1}-\Sigma_{1} \subseteq V_{2}-\Sigma_{2}, \Sigma_{1} \subseteq \Sigma_{2}$, and for some integer $\ell \geq 1$, the conditions $X$ is in $V_{1}$ and $X \Rightarrow \alpha$ in $G_{2}$ imply

(i) $X \underset{\text { tnt }}{\Rightarrow} \propto$ in $G_{2}$, and

(ii) $x \rightarrow \alpha$ is in $P_{1}$.

$$
\text { Then } \mathcal{L}_{s}\left(G_{2}, \Rightarrow \subseteq \mathcal{L}_{s}\left(G_{1}, \Rightarrow\right)\right.
$$

Proof: Without loss of generality we may assume $X \rightarrow \alpha$ is in $P_{1}$ iff $X \underset{\text { tnt }}{\Rightarrow} \alpha$ in $G_{2}$. For assuming otherwise we consider the subgrammar $G_{1}^{\prime \prime}$ of $G_{1}$ defined by this condition. clearly $\mathcal{Z}_{s}\left(G_{1}^{\prime \prime} \Rightarrow\right) \subseteq \mathcal{Z}_{s}\left(G_{1} \Rightarrow\right)$ and it is sufficient to prove that $\mathcal{Z}_{s}\left(G_{2}, \Rightarrow \subseteq \mathcal{Z}_{s}\left(G_{1}, \Rightarrow\right)\right.$.

Consider an arbitrary interpretation $G_{2}^{\prime}=\left(V_{2}^{\prime}, E_{2}^{\prime}, P_{2}^{\prime}, S^{\prime}\right) \mathbb{S}_{S} G_{2}\left(\mu_{2}\right)$. Define $G_{1}^{i}=\left(V_{j}^{\prime}, \Sigma_{j}^{i}, P_{j}, S\right) \Delta_{s} G_{1}\left(\mu_{1}\right)$ by

$$
x^{\prime} \rightarrow \alpha^{\prime} \text { is in } P_{1}^{\prime} \text { iff } X^{\prime} \Rightarrow^{2} \alpha^{\prime} \text { in } G_{2}^{\prime} \text {. }
$$

clearly $V_{1}^{\prime}-\Sigma_{1}^{\prime} \subseteq V_{2}^{\prime}-\Sigma_{2}^{\prime}$ and $\Sigma_{1}^{\prime} \subseteq \Sigma_{2}^{\prime}$, hence $\mu_{1}(x)=\mu_{2}(x)$, for all $x$ in $V_{1}$. Furthermore $P_{1} \subseteq \mu_{1}\left(P_{1}\right)$ as desired. We now demonstrate that $L\left(G_{1}^{\prime}, \Rightarrow\right)=L\left(G_{2}^{1}, \Rightarrow\right)$. 
Let $x^{\prime}$ be in $L\left(G_{2}^{\prime}, \Rightarrow\right)$, that is, $S^{\prime} \Rightarrow^{k} x^{\prime}$ in $\Sigma_{2}^{\prime *}$. Now $k=m e$, for some $m>1$, since otherwise:

$S^{\prime} \Rightarrow^{m l} y^{\prime} \Rightarrow^{t} x^{\prime} \Rightarrow^{l-t} z^{\prime}$ with $y^{\prime}$ and $z^{\prime}$ in $V_{1}^{\prime *}$,

for some $t, 1 \leq t<l$.

which leads to a contradiction as in the previous lemma. Thus $S^{\prime} \Rightarrow{ }^{k} x^{\prime}$ in $G_{2}^{\prime}$ implies $S^{\prime} \Rightarrow m l x^{\prime}$ in $G_{2}^{\prime}$ hence

$S^{\prime} \Rightarrow x^{\prime}$ in $G_{1}^{\prime}$ by construction.

That is, $x^{\prime}$ is in $L\left(G_{j}^{\prime}, \Rightarrow\right)$.

Conversely, consider any $x^{\prime}$ in $L\left(G_{j}^{\prime} \Rightarrow\right)$, that is,

$S^{\prime} \Rightarrow x^{\prime}$ in $G_{j}^{\prime}$,

again by construction we have

$S^{\prime} \Rightarrow m e x^{\prime}$ in $G_{2}^{\prime}$

and therefore $x^{\prime}$ is in $L\left(G_{2}^{\prime}, \Rightarrow\right)$.

As an application of these two simulation lemmas consider the following example.

Let $G_{1}$ be defined by:

$S \rightarrow S S ; S \rightarrow S ; S \rightarrow a ; a \rightarrow S S ; a \rightarrow S ; a \rightarrow a$

and $G_{2}$ by:

$S \rightarrow A ; A \rightarrow S S ; A \rightarrow S ; A \rightarrow a ; a \rightarrow A$.

Then $G_{1}$ and $G_{2}$ are form equivalent.

Since $S \Rightarrow A \Rightarrow S S ; S \Rightarrow A \Rightarrow S ; S \Rightarrow A \Rightarrow a$;

$a \Rightarrow A \Rightarrow S S ; a \Rightarrow A \Rightarrow S ; a \Rightarrow A \Rightarrow a$

in $G_{2}$ for each production in $G_{1}$, we have $\mathcal{L}_{s}\left(G_{1}, \Rightarrow \subseteq \mathcal{L}_{s}\left(G_{2}, \Rightarrow\right)\right.$. Conversely these are the only length 2 derivations from $S$ and a in $G_{2}$, and they are tnt-derivations, hence $\mathcal{L}_{\mathrm{s}}\left(G_{2}, \Rightarrow\right) \subseteq \mathcal{L}_{\mathrm{s}}\left(G_{1}, \Rightarrow\right)$, giving form equivalence.

We can generalize Lemmas $1.9,1.10$ and 1.11 to the case of ETOL forms. However we only state, without proof, the correspondents of Lemmas 1.10 and 1.11 .

\section{Lemma $1.12--$ Expansion Simuzation Lemma}

Suppose $G_{i}=\left(V_{i}, \Sigma_{i}, P_{i}, 1, \ldots, P_{i, n}, s\right), i=1,2$ are two $n$-tabled ETOL forms such that $V_{1}-\Sigma_{1} \subseteq V_{2}-\Sigma_{2}, \Sigma_{1} \subseteq \Sigma_{2}$ and for some integers $\ell_{1}, \ldots, \ell_{n} \geq 1$,

$\quad x \rightarrow \alpha$ in $P_{1, j}$ implies $x \stackrel{\ell_{j}}{\Rightarrow}$ a in $P_{2, j}$.

Then $\mathcal{L}_{s}\left(G_{1}, \Rightarrow\right) \subseteq \mathcal{L}_{s}\left(G_{2}, \Rightarrow\right)$. 
Lemma 1.13 - - Contraction SimuZation Lemma

Suppose $G_{i}=\left(V_{i}, \Sigma_{i}, P_{i, 1}, \ldots, P_{i, n}, S\right), i=1,2$ are two $n$-tabled ETOL forms such that $V_{1}-\Sigma_{1} \subseteq V_{2}-\Sigma_{2}, \Sigma_{1} \subseteq \Sigma_{2}$ and for some integers $\ell_{1}, \ldots, \ell_{n} \geq 1$, the conditions $x$ is in $V_{1}$ and $x \Rightarrow^{\ell_{j}}$ a in $P_{2, j}$ imply:

(i) $x \underset{t n t}{\Rightarrow}{ }^{l} j$ a in $P_{2, j}$, and

(ii) $X \rightarrow \alpha$ is in $P_{1, j}$.

Then $\mathcal{L}_{\mathrm{s}}\left(\mathrm{G}_{2}, \Rightarrow \subseteq \mathcal{L}_{\mathrm{s}}\left(\mathrm{G}_{1}, \Rightarrow\right)\right.$.

Since the simulation is localized to particular tables the proofs of Lemmas 1.12 and 1.13 can be reduced to $n$ applications of Lemmas 1.10 and 1.11 , respectively. 


\section{III.2 Reduction Theorems}

Our aim in this section is to demonstrate that most transformations that may be applied to EOL grammars or ETOL grammars preserving their languages can be applied to EOL or ETOL forms preserving their language families. There are however two exceptions, namely, in general the synchronization transformation and the propagating transformation do not preserve form equivalence.

The transformations considered are not only of interest in their own right but also are useful in the following two sections on completeness and goodness.

\section{III.2.1 Some Preliminary Reduction and Non-Reduction Results}

We say an ETOL grammar (and form) $G=\left(V, \Sigma, P_{1}, \ldots, P_{n}, S\right)$ is reduced if every symbol appears in some word derivable from $S$. We say $G$ is separated if for all $P_{i}, X \rightarrow \alpha$ in $P_{i}$ implies either $\alpha$ is in $\Sigma u(V-\Sigma)^{*}$ if $X$ is in $V-\Sigma$ or $\alpha$ is in $(V-\Sigma)^{+}$if $X$ is in $\Sigma$. We say $G$ is synchronized if, for each a in $\Sigma, a \Rightarrow^{+} \alpha$ in $G$ implies $\alpha$ is not in $\Sigma^{*}$. $G$ is short if for all $P_{i}, X \rightarrow \alpha$ in $P_{i}$ implies $|\alpha| \leq 2$ and $G$ is binary if each production in each table is of one of the types: $A \rightarrow \lambda$; $\rightarrow a$; $A \rightarrow B ; A \rightarrow B C ; a \rightarrow A$; where $A, B, C$ are in $V-\Sigma$ and $a$ is in $\Sigma$.

Our first result is stated without proof, namely,

Lemma 2.1

For every ETOL form $G$ a form-equivalent reduced ETOL form $H$ can be constructed.

Secondly, we demonstrate that separability preserves formequivalence.

Lemma 2.2

For every $E T O L$ form $G=\left(V, \Sigma, P_{1}, \ldots, P_{n}, S\right)$ a form-equivalent separated ETOL form $F=\left(U, \Sigma, Q_{1}, \ldots, Q_{n}, S\right)$ can be constructed.

Proof: Let $U=V \cup V^{\prime}$, where $V^{\prime}=\{\bar{X}: X$ in $V\} \cup\{\bar{\lambda}\}$ is a set of new nonterminals and define $\overline{x y}=\bar{x} \bar{y}$ for $x, y$ in $v^{+}$. For all $i, 1 \leq i \leq n$, 1et

$$
Q_{i}=\left\{X \rightarrow \bar{\alpha}: X \rightarrow \alpha \text { is in } P_{i}\right\} \cup\left\{\bar{X} \rightarrow X: \bar{X} \text { is in } V^{\prime}\right\} \text {. }
$$


Observe that for each production $X \rightarrow \alpha$ in $P_{i}$ there is a derivation $X \Rightarrow \bar{\alpha} \Rightarrow \alpha$ in $Q_{i}, 1 \leq i \leq n$, hence $F$ expansion simulates $G$ therefore $\mathcal{L}_{\mathrm{s}}(G, \Rightarrow) \subseteq \mathcal{L}_{\mathrm{S}}(F, \Rightarrow)$ by Lemma 1.12. Conversely, G contraction simulates $F$, hence by Lemma 1.13 we have $\mathcal{Z}_{S}\left(F, \Rightarrow \subseteq \mathcal{L}_{s}(G, \Rightarrow)\right.$. Finally note that $F$ is indeed separated.

The next lemma, the shortening lemma, enables us to reach one of the goals of this section, namely every ETOL form has a formequivalent binary ETOL form. For an arbitrary ETOL form $G=\left(V, \Sigma, P_{j}, \ldots P_{n}, S\right)$ let $\max (G)=\max \left(\left\{|\alpha|: X \rightarrow \alpha\right.\right.$ is in $P_{i}$, for some $i, 1 \leq i \leq n\}$ ).

\section{Lemma 2.3}

For every $E T O L$ form $G=\left(V, \Sigma, P_{1}, \ldots, P_{n}, S\right)$ a form-equivalent short ETOL form $F=\left(U, \Sigma, Q_{1}, \ldots, Q_{n}, S\right)$ can be constructed.

Proof: If $\max (G) \leq 2$ then $G$ is already short and there is nothing to be shown, therefore consider $\max (G)=t \geq 3$. We construct $H=\left(W, \Sigma, R_{1}, \ldots, R_{n}, S\right)$ such that $\operatorname{maxr}(H)<\operatorname{maxr}(G)$ and $H$ is formequivalent to $G$. By iterating the construction the desired $F$ is finally obtained.

For every production $p: X \rightarrow \alpha$ in $P_{i}$, for every $i, 1 \leq i \leq n$, let $[p, 1, i]$ and $[p, 2, i]$ be two new nonterminals and $W_{1}=\{[p, 1, i]$, $[p, 2, i]: p$ is in $\left.P_{i}, 1 \leq i \leq n\right\}$. Let $W=W_{1} \cup V$ and define the $R_{j}$ as follows:

For all $i, 1 \leq i \leq n$,

$$
\begin{aligned}
R_{i}= & \left\{X \rightarrow[p, 1, i],[p, 1, i] \rightarrow \alpha: p: X \rightarrow \alpha \text { is in } P_{i} \text { and }|\alpha|<t\right\} \\
& \left\{X \rightarrow[p, 1, i][p, 2, i],[p, 1, i] \rightarrow A_{1} \ldots A_{t-1},[p, 2, i] \rightarrow A_{t}:\right. \\
& \left.p: X \rightarrow A_{1} \ldots A_{t} \text { is in } P_{i} \text { and } A_{j} \text { is in } V, 1 \leq j \leq t\right\} .
\end{aligned}
$$

observe that for all $i, 1 \leq i \leq n$, for all $p: X \rightarrow \alpha$ in $P_{i}$, there is:

either a derivation $x \Rightarrow[p, 1, i] \Rightarrow \alpha$ in $R_{i}$

or a derivation $X \Rightarrow[p, 1, i][p, 2, i] \Rightarrow \alpha$ in $R_{i}$.

Hence $H$ expansion simulates $G$ and $\mathcal{L}_{s}(G, \Rightarrow) \subseteq \mathcal{L}_{s}(H, \Rightarrow)$ and also $G$ contraction simulates $H$ giving the reverse inclusion.

Finally note that $\max (H)<\max (G)$, therefore we may iterate the construction until we have obtained an $F$ with maxr $(F)=2$. Clearly $F$ and $G$ will be form equivalent.

We are now in a position to state and prove our first theorem. 
Theorem 2.4

For every $E T O L$ form $G=\left(V, \Sigma, P_{1}, \ldots, P_{n}, S\right)$ a form-equivalent binary ETOL form $F=\left(U, \Sigma, Q_{1}, \ldots, Q_{n}, S\right)$ can be constructed.

Proof: We may assume by Lemmas 2.2 and 2.3 that $G$ is both separated and short, hence the only productions not of the correct type are those of type:

$$
p: a \rightarrow A B \text {, a in } \Sigma \text { and } A, B \text { in } V-\Sigma \text {. }
$$

We therefore carry out a construction similar to that given in Lemma 2.3.

Let $U=V \cup\left\{[p, i]: p\right.$ is a production in $\left.P_{i}, 1 \leq i \leq n\right\}$ and for all $i, 1 \leq i \leq n$ define $Q_{i}$ by:

$$
Q_{j}=\left\{X \rightarrow[p, i],[p, i] \rightarrow \alpha: p: X \rightarrow \alpha \text { is in } P_{i}\right\} .
$$

Clearly by Lemmas 1.12 and $1.13 \mathcal{L}_{\mathrm{s}}(F, \Rightarrow)=\mathcal{L}_{\mathrm{s}}(G, \Rightarrow)$ and, moreover, the productions in $F$ are all binary.

We now turn to the two non-reduction results already mentioned. Both of them use terminal forcing to obtains a counter-example, that is a grammar $G$ is terminal forcing if there is a word $x$ in its language whose derivation includes some other word $y$ in the language (you can't have $x$ without having some other word).

\section{Theorem 2.5}

Let $G=(\{S, a, b\},\{a, b\},\{S \rightarrow a ; a \rightarrow b ; b \rightarrow b\}, S)$ be an EOL form. Then there is no synchronized EOL form $F$ with $\mathcal{L}_{s}(F, \Rightarrow)=\mathcal{L}_{s}(G, \Rightarrow)$.

Proof: Note that for every $G^{\prime} \vec{s} G, L\left(G^{\prime}, \Rightarrow\right)$ contains at least two words, in fact symbols. This follows by the definition of s-interpretation and EOL grammars and the relationship between the form derivations and interpretation derivations. Now assume $F=(U, \Delta, Q, A)$ is a synchronized EOL form, which is form-equivalent to $G$.

Immediately, since $F$ is synchronized, whenever

$$
A \Rightarrow^{+} x \text { in } \Delta^{+} \text {in } F
$$

then for all $\alpha$, such that

$$
x \stackrel{+}{\Rightarrow} \alpha \text { in } F
$$

$\alpha$ is not in $\Delta^{*}$.

Consider an isolating interpretation $F^{\prime}$ of such a derivation, then $L\left(F^{\prime}, \Rightarrow\right)$ consists of one word and hence is not in $\mathcal{L}_{s}(G, \Rightarrow)$. This provides a contradiction to the assumption that $\mathscr{L}_{S}(G, \Rightarrow)=\mathcal{Z}_{s}(F, \Rightarrow)$. 
However, it turns out that in the case of synchronized ETOL forms we can find an $F$ form-equivalent to the $G$ of Theorem 2.5.

\section{Theorem 2.6}

Let $G=(\{S, a, b\},\{a, b\},\{S \rightarrow a ; a \rightarrow b ; b \rightarrow b\}, S)$ be an $E O L$ form and $F=\left(\{S, N, A, B, a, b\},\{a, b\}, P_{1}, P_{2}, S\right)$ be a synchronized ETOL form where

$P_{1}=\{S \rightarrow A ; A \rightarrow B ; B \rightarrow B ; a \rightarrow N ; b \rightarrow N ; N \rightarrow N\}$ and

$P_{2}=\{A \rightarrow a ; B \rightarrow b ; S \rightarrow N ; a \rightarrow N ; b \rightarrow N ; N \rightarrow N\}$.

Then $\mathcal{L}_{s}(G, \Rightarrow)=\mathcal{L}_{s}(F, \Rightarrow)$.

Proof: First observe that $F$ is indeed synchronized.

(i) $\mathcal{Z}_{s}(G, \Rightarrow) \subseteq \mathcal{L},(F, \Rightarrow)$.

Consider an arbitrary interpretation $G^{\prime} \underset{S}{\Delta} G(\mu)$ where

$G^{\prime}=(\{S\} \cup \Sigma, \Sigma, P, S)$ without any loss of generality. Construct

$F^{\prime}=\left(V, \Sigma, P_{1}^{\prime}, P_{2}^{\prime}, S\right) \underset{S}{\Delta} F\left(\mu^{\prime}\right)$ as follows: Let $V=\{S, N\} \cup \Sigma \cup\left\{a^{\prime}:\right.$ a in $\Sigma$, where the a are new nonterminals,

$$
\begin{aligned}
P_{1}^{\prime}= & \left\{S \rightarrow a^{\prime}: S \rightarrow a \text { in } P\right\} \\
\cup & \left\{C^{\prime} \rightarrow d^{\prime}: c \rightarrow d \text { in } P\right\} \\
& \cup\{a \rightarrow N: a \text { in } \Sigma\} \cup\{N \rightarrow N\} \\
P_{2}^{\prime}=\left\{a^{\prime} \rightarrow a: a \text { in } \Sigma\right\} & \cup\{a \rightarrow N ; a \text { in } \Sigma\} \\
& \cup\{S \rightarrow N ; N \rightarrow N\},
\end{aligned}
$$

and $\mu^{\prime}$ is defined by:

$$
\mu^{\prime}(S)=S, \mu^{\prime}(N)=N \text {, }
$$

for al1 a in $\Sigma, \mu^{\prime}(a)=\mu(a)$,

$\mu^{\prime}(A)=\left\{c^{\prime}: c\right.$ in $\left.\mu(a)\right\}$ and

$\mu^{\prime}(B)=\left\{c^{\prime}: c\right.$ in $\left.\mu(b)\right\}$.

We claim that $L\left(F^{\prime}, \Rightarrow\right)=L\left(G^{\prime}, \Rightarrow\right)$.

Without belaboring the details observe that

$$
S \Rightarrow C \text { in } G^{\prime}
$$

implies $S^{+} C^{\prime}$ in $P_{j}^{\prime}$ and $C^{\prime} \rightarrow C^{\prime}$ in $P_{2}^{\prime}$, hence

$$
S \Rightarrow c \text { in } F^{\prime} \text {. }
$$

Conversely, any terminating derivation in $F^{\prime}$ is composed of a sequence of applications of $P_{j}$ followed by an application of $P_{2}^{\prime}$. It remains to note that occurrences of terminals in a derivation in $G^{\prime}$ are replaced by their primed equivalents in the corresponding derivation in $F^{\prime}$. (ii) $\underline{\mathcal{L}}_{\mathrm{s}}(\mathrm{F}, \Rightarrow) \subseteq \mathcal{Z}_{\mathrm{S}}(\mathrm{G}, \Rightarrow)$.

Consider an arbitrary interpretation $F^{\prime}=\left(V, \Sigma, P^{\prime}, S\right) \triangle_{S} F(\mu)$. We construct a $G^{\prime}=(\{S\} \cup \Sigma, P, S) \underset{S}{\Delta} G\left(\mu^{\prime}\right)$ such that $L\left(G^{\prime}, \Rightarrow\right)=L\left(F^{\prime}, \Rightarrow\right)$.

Without loss of generality we may assume that $F^{\prime}$ is reduced in which case $L\left(F^{\prime}, \Rightarrow\right)=\Sigma_{a} \cup \Sigma_{b}=\Sigma$, where $\Sigma_{a} \subseteq \mu(a)$ and $\Sigma_{b} \subseteq \mu(b)$, and 
moreover $\Sigma_{a} \neq \emptyset \neq \Sigma_{b}$. Hence define $P$ as:

$P=\left\{S \rightarrow a:\right.$ for all a in $\left.\Sigma_{a}\right\}$

b $\{a \rightarrow b, b \rightarrow b$ : for $a\rceil 1$ a in $\Sigma_{a}, b$ in $\left.\Sigma_{b}\right\}$.

clearly $L\left(G^{\prime}, \Rightarrow\right)=L\left(F^{\prime}, \Rightarrow\right)$ and $G^{\prime} A_{S} G$. Hence the result.

To obtain the above result we have synchronized $G$ in a different way than is usual for EOL or ETOL forms. The key idea is not only to introduce marked variants of the terminals but also to provide a new table in which the marked terminals become themselves once more. In the usual technique the marked-terminal-to-terminal productions are added to all tables.

However there are EOL forms for which the above technique does not apply.

\section{Theorem 2.7}

Let $G=(\{S, a\},\{a\},\{S \rightarrow a ; a \rightarrow a a\}, S)$ be an EOL form. Then no synchronized ETOL form is form-equivalent to $G$.

Proof: Assume $F=\left(V, \Sigma, P_{1}, \ldots, P_{n}, S\right)$ is a synchronized ETOL form which is form-equivalent to $G$. Then there exists

$F^{\prime}=\left(V^{\prime},\{a\}, P_{1}^{\prime}, \ldots, P_{n}^{\prime}, S^{\prime}\right) \bigotimes_{S} F$ with $L\left(F^{\prime}, \Rightarrow\right)=L(G, \Rightarrow)$. Consider a derivation of $a^{4}$ in $F^{\prime}$

$$
S^{\prime} \Rightarrow \alpha_{1} \Rightarrow . \ldots \alpha_{r}=a^{4} \text {, for some } r \geq 1 \text {. }
$$

since $F$ is synchronized, $\alpha_{i}$ is in $\left(V^{\prime}-\{a\}\right)^{+}$for $l \leq i \leq r$. Hence this derivation can be isolated in an interpretation $F^{\prime \prime}$ of $F^{\prime}$ in such a way that

(i) $P_{i} \leq P_{i}^{\prime \prime}, 1 \leq i \leq n$, and

(ii) $S^{\prime} \Rightarrow \alpha_{1}^{\prime \prime} \Rightarrow \ldots \Rightarrow \alpha_{r}^{\prime \prime}=$ abab is the result of the isolated derivation, and

$$
L\left(F^{\prime \prime}, \Rightarrow\right)=L\left(F^{\prime}, \Rightarrow\right) \cup\{a b a b\}
$$

$$
=\left\{a^{2^{p}}: p \geq 0\right\} \cup\{a b a b\} \text {. }
$$

clearly $L\left(F^{\prime \prime}, \Rightarrow\right)$ is not in $\mathcal{L}_{S}(G, \Rightarrow)$, consequently $\mathcal{L}_{S}(F, \Rightarrow) \neq \mathcal{L}_{S}(G, \Rightarrow)$.

Whether in fact the ETOL synchronization technique can be applied only to finite EOL forms remains an open question.

We now turn to the second promised non-reduction result. 


\section{Theorem 2.8}

Let $G=(\{S, a, b\},\{a, b\},\{S \rightarrow a ; a \rightarrow a b b a ; b \rightarrow \lambda\}, S)$. Then no propagating ETOL form is form-equivalent to $G$.

Proof: Assume to the contrary that $F=\left(V, \Sigma, P_{\eta}, \ldots, P_{n}, S\right)$ is a propagating ETOL form form-equivalent to $G$.

Consider $F^{\prime}=\left(V^{\prime},\{a, b\}, P_{j}^{\prime}, \ldots, P_{n}^{\prime}, S^{\prime}\right) \underset{S}{\Delta} F$ such that $L\left(F^{\prime} \Rightarrow\right)=L(G, \Rightarrow)$ and a shortest derivation in $F^{\prime}$ of the form (*) $\quad S^{\prime} \Rightarrow \alpha_{1} \Rightarrow \ldots \Rightarrow a_{r}=a b b a$.

That is, there is no shorter derivation for abba in $F^{\prime}$. Since it is a shortest derivation and $F^{\prime}$ is propagating at most one of $\alpha_{1}, \ldots, \alpha_{r-1}$ is terminal and in this case equal to a.

Consider an isolating interpretation $F^{\prime \prime}$ of $F^{\prime}$ such that (*) is the only possible prefix of any longer derivation in F" and if a occurs in (*) it remains as a.

If $F^{\prime \prime}$ is finite, a contradiction has been obtained since for al1 $G^{*} \triangle_{S} G, G^{\prime}$ is infinite. On the other hand if $F^{\prime \prime}$ is infinite, since it is propagating abbaaba cannot be derived from abba. This also gives a contradiction since $L(G, \Rightarrow)$ - \{abbaabba $\}$ is clearly not in $\mathcal{L}_{\mathrm{s}}(G, \Rightarrow)$. Consequently $\mathcal{L}_{\mathrm{s}}(\mathrm{F}, \Rightarrow) \neq \mathcal{L}_{\mathrm{s}}(G, \Rightarrow)$, hence the result.

\section{III.2.2 Propagating and Two-Table Normal Forms}

We first demonstrate that in the case that an ETOL form is synchronized the propagating transformation can be applied successfully. Secondly, we demonstrate that the 2-table normal form result for ETOL grammars carries over to ETOL forms using however a different transformation technique.

\section{Notational Convention}

We will refer to synchronized EOL and ETOL forms as synchro-EOL and -ETOL forms in the following. Moreover if $G=(V, \Sigma, P, \ldots, P, S)$ is a synchro-ETOL form we assume the presence of a universally designated blocking symbol $N$ whose only production $N \rightarrow N$ is in every table and the presence of productions $a \rightarrow N$ for all a in $\Sigma$ in all $P_{i}, 1 \leq i \leq n$. Hence typically a synchro-EOL form can be specified by giving the sentence symbol and the productions for the nonterminals excluding $\mathrm{N}$, as is done for context-free grammars. Moreover, interpretations will onty deal with non-N symbols. 
Theorem 2.9

For every synchro-ETOL form $G=\left(V, \Sigma, P_{1}, \ldots, P_{n}, S\right)$ a formequivalent propagating synchro-ETOL form $F=\left(U, \Sigma, Q_{1}, \ldots, Q_{n},[S, \emptyset]\right)$ can be constructed.

Proof: We will first give the construction. We may assume by the results of the previous section that $G$ is binary. [The proofs can be slightly modified to take into account our conventions for synchroETOL forms.] We also assume without loss of generality that $A \rightarrow N$ is in $P_{i}$ for all $A$ in $V-\Sigma$ and all $i, 1 \leq i \leq n$. Hence the productions of each $P_{i}$ are of the following types:

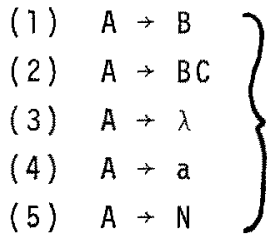

For the purposes of the construction we need the following notion. For $X \subseteq V-\Sigma$ and for all $i, 1 \leq i \leq n$, let $P_{j}(X)=\{Y: Y \subseteq V-\Sigma$, there exists $\alpha, \beta$ in $(V-\Sigma) *$ such that $\alpha \Rightarrow \beta$ in $P_{i}, X=\operatorname{alph}(\alpha)$ and $\left.Y=\operatorname{alph}(\beta)\right\}$. Let $U=\Sigma U\{[A, X]: A$ is in $V-\Sigma$ and $\left.X \subseteq V-\sum\right\}$ and for all $i, 1 \leq i \leq n, Q_{i}$ contains:

for a $11 X \subseteq V-\Sigma$ and $Y$ in $P_{j}(X)$

(1') $[A, X] \rightarrow[B, Y]$, where $A \rightarrow B$ is in $P_{j}$,

$\left.\begin{array}{l}\left(2^{\prime}\right)[A, X] \rightarrow[B, Y][C, Y], \\ \left(2^{\prime \prime}\right) \quad[A, X] \rightarrow[B, Y \cup[C\}], \text { if } C \Rightarrow^{+} \lambda \\ \left(2^{\prime \prime \prime}\right)[A, X] \rightarrow[C, Y \cup\{B\}], \text { if } B \Rightarrow^{+} \lambda\end{array}\right\}$ where $A \rightarrow B C$ is in $P_{i}$,

$\left(2^{\prime \prime \prime}\right)[A, X] \rightarrow[C, Y \cup\{B\}]$, if $B \Rightarrow^{+} \lambda$

(4') $[A, X] \rightarrow a$, if $\emptyset$ is in $P_{i}(X)$ and $A \rightarrow$ a is in $P_{j}$,

(5') $[A, X] \rightarrow N$, for a 1$][A, X]$ in $U$.

We now prove form-equivalence in two parts.

(i) $\underline{\mathcal{L}}_{s}(G, \Rightarrow) \subseteq \mathcal{L}_{s}(F, \Rightarrow)$.

Consider an arbitrary. interpretation

$G^{\prime}=\left(V^{\prime}, \Sigma^{\prime}, P_{j}^{\prime}, \ldots, P_{n}^{\prime}, S^{\prime}\right) \triangle_{S} G(\mu)$. We will construct an

$F^{\prime}=\left(U^{\prime}, \Sigma^{\prime}, Q_{j}^{\prime}, \ldots, Q_{n}^{\prime},\left[S^{\prime}, \emptyset\right]\right)$ such that $F^{\prime} \triangle_{S} F\left(\mu^{\prime}\right)$ and $L\left(F^{\prime}, \Rightarrow\right)=$

$L\left(G^{\prime}, \Rightarrow\right)$. Define $\mu^{\prime}$ by:

$\mu^{\prime}(a)=\mu(a)$, for a 11 a in $\Sigma$, and

$\mu^{\prime}([A, X])=\{[B, X]: B$ is in $\mu(A)\}$, for all $[A, X]$ in $U$.

For all $i, 1 \leq i \leq n$, construct $Q_{i}^{i}$ from $P_{i}$ as follows:

(a) for each type 1 production $A^{\prime} \rightarrow B^{\prime}$ in $\mu(A \rightarrow B)$ take all productions $\left[A^{\prime}, X\right] \rightarrow\left[B^{\prime}, Y\right]$ for all $X \subseteq V-\sum$ and $Y$ in $R_{i}(X)$, where $R_{i}=\mu^{-1}\left(P_{i}^{1}\right)$. 
(b) for each type 2 production $A^{\prime} \rightarrow B^{\prime} C^{\prime}$ in $\mu(A \rightarrow B C)$ take similarly $\left[A^{\prime}, X\right] \rightarrow\left[B^{\prime}, Y\right]\left[C^{\prime}, Y\right]$, if $C \Rightarrow^{*} \lambda$ then $\left[A^{\prime}, X\right] \rightarrow\left[B^{\prime}, Y \cup\{C\}\right]$ and if $B \Rightarrow^{*} \lambda$ then $\left[A^{\prime}, X\right] \rightarrow\left[C^{\prime}, Y \cup[B]\right]$, where $X$ and $Y$ are as in $(1)$, and

(c) for each type 4 production $A^{\prime} \rightarrow a^{\prime}$ in $\mu\left(A \rightarrow\right.$ a) take $\left[A^{\prime}, X\right] \rightarrow a^{\prime}$ iff $\emptyset$ is in $R_{i}(X)$, where $X$ is as in (1)

(d) for each type 5 production $A^{\prime} \rightarrow N$ in $\mu(A \rightarrow N)$ take $\left[A^{\prime}, X\right] \rightarrow N$, for a $11 X \subseteq V-\Sigma$.

That $L\left(F^{\prime} \Rightarrow\right)=L\left(G^{\prime} \Rightarrow\right)$ follows since we have used essentially the standard construction, and furthermore we have $Q_{i}^{\prime} \subseteq \mu^{\prime}\left(Q_{i}\right)$, hence $F^{\prime} \bigotimes_{s} F\left(\mu^{\prime}\right)$. This completes the first part of the theorem.

(ii) $\mathcal{L}_{\mathrm{s}}(\mathrm{F,g}) \subseteq \mathcal{L}_{\mathrm{s}}(\mathrm{G}, \Rightarrow)$.

To illustrate the technique of the proof we will restrict ourselves to the case $n=1$, that is, $F$ and $G$ are $E O L$ forms. The extension to the case $n>1$ is left to the reader. Consider then $F=(U, \Sigma, Q, S)$ and $G=(V, \Sigma, P, S)$. When we take an arbitrary $F^{\prime} \Delta_{S} F(\mu)$, where $F^{\prime}=\left(U^{\prime}, \Sigma^{\prime}, Q^{\prime}, S^{\prime}\right)$ it is straightforward to construct $G^{\prime}$ if $Q^{\prime}$ contains no productions $q: D^{\prime} \rightarrow E^{\prime}$, which are interpretations of:

$$
[A, X]+[B, Y \cup\{C\}] \text { or }[A, X] \rightarrow[C, Y \cup\{B\}] \text {. }
$$

In this case each production in $Q^{\prime}$ is an interpretation of a production not only in $Q$ but also in $P$, therefore take $P^{\prime}=Q^{\prime}$.

In general, of course, we cannot assume $Q^{\prime}$ fulfills this condition, therefore we also add to $\mathrm{P}^{\prime}$ productions of the type:

$$
D^{\prime} \rightarrow E^{\prime} C_{q} \text { or } D^{\prime} \rightarrow B_{q} E^{\prime}
$$

where $C_{q}$ and $B_{q}$ derive only the empty word.

Construct $G^{\prime} \triangle_{S} G\left(H^{\prime}\right)$ where $G^{\prime}=\left(V^{\prime}, \Sigma^{\prime}, P^{\prime}, S^{\prime}\right)$ as follows:

First define $\mu^{\prime}$ by:

$$
\begin{aligned}
\mu^{\prime}(a) & =\mu(a), \text { for a } 11 \text { a in } \Sigma \text {, and } \\
\mu^{\prime}(A) & =\bigcup_{X \subseteq V-\Sigma} \mu([A, X]) \\
& \cup\left\{A_{q}: q \text { is a production of } Q^{\prime}\right\},
\end{aligned}
$$

and let $V^{\prime}=\mu^{\prime}(V)$. Note that $\mu([S, \theta]) \subseteq \mu^{\prime}(S)$ and hence $S^{\prime}$ is in $\mu^{\prime}(S)$.

Second, construct $P^{\prime}$ by taking into $P^{\prime}$ all productions in $Q^{\prime}$ which are interpretations of $1^{\prime}, 2^{\prime}, 4^{\prime}$ and $5^{\prime}$. Clearly by definition of $\mu^{\prime}$ these productions are indeed interpretations of $1,2,4$ and 5 , respectively. It remains to treat productions which stem from $2^{\prime \prime}$ and $2^{\text {"1. }}$

For each production $q: D^{\prime} \rightarrow E^{\prime}$ in $\mu([A, X] \rightarrow[B, Y u\{C\}])$ (that is, from a type $2 "$ production), take $D^{\prime} \rightarrow E^{\prime} C_{q}$ into $P^{\prime}$ together with all productions in $1235(P, q)$. We define $1235(P, q)$ as 


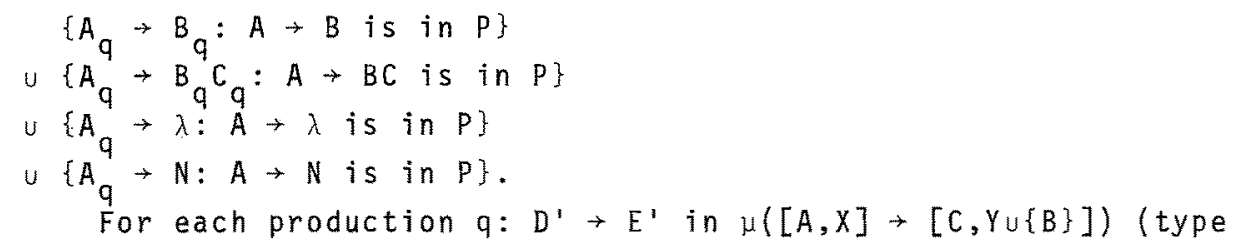
$2^{\prime \prime \prime}$ productions), take

$$
D^{\prime} \rightarrow B_{q} E^{\prime} \text { into } p^{\prime}
$$

together with all productions $1235(P, q)$.

Note immediately that $D^{\prime} \rightarrow E^{\prime} C_{q}$ is in $\mu^{\prime}(P), D^{\prime} \rightarrow B_{q} E^{\prime}$ is in $\mu^{\prime}(P)$ and $1235(P, q) \subseteq \mu^{\prime}(P)$. Hence $p^{\prime} \subseteq \mu^{\prime}(P)$ as desired giving $G^{\prime} \underset{S}{\Delta} G^{\prime}\left(\mu^{\prime}\right)$. It remains to prove $L\left(G^{\prime}, \Rightarrow\right)=L\left(F^{\prime} \Rightarrow\right)$.

(iia) $L\left(G^{\prime}, \Rightarrow\right) \subseteq L\left(F^{\prime}, \Rightarrow\right)$.

\section{Suppose}

(*) $\quad S^{\prime}=\alpha_{1} \Rightarrow \ldots \alpha_{m}=x$ in $\Sigma^{\prime} *$ in $G^{\prime}$.

Note that subscripted nonterminals (that is, nonterminals of the type $A_{q}$ ) only generate the empty word. Thus a production $D \rightarrow$ a appearing in $(*)$ is one of three types:

(1) D and $\alpha$ are both unsubscripted,

(2) D is unsubscripted and a contains a subscripted nonterminal,

(3) D and a are both subscripted.

By eliminating productions of type (3), dropping the subscripted nonterminal in those of type (2) and carrying over productions of type (1) a derivation

$$
S^{\prime}=\alpha_{j}^{\prime} \Rightarrow \ldots \Rightarrow \alpha_{m}^{\prime}=x \text { in } F^{\prime}
$$

is obtained.

\section{(iib) $L\left(F^{\prime}, \Rightarrow\right) \subseteq L\left(G^{\prime}, \Rightarrow\right)$.}

\section{Suppose}

(*) $\quad S^{\prime}=\alpha_{1} \Rightarrow \ldots \Rightarrow \alpha_{m} \Rightarrow x$ in $\Sigma^{\prime} *$ in $F^{\prime}$.

Then we claim. there exists a derivation in $G^{\prime}$

$(* \star) \quad S^{\prime}=\alpha_{1}^{\prime} \Rightarrow \ldots \alpha_{m}^{\prime}$

such that

if $\quad \alpha_{i}=D_{1} \ldots D_{k}$ with $D_{j}$ in $\mu\left(\left[A_{j}, X_{j}\right]\right), 1 \leq j \leq k$

then $\quad \alpha_{j}^{\prime}=B_{0} D_{1} \ldots D_{k} \beta_{k}$

where $\beta_{0} \ldots \beta_{k}$ consists of only subscripted nonterminals and a)ph $\left(\operatorname{strip}\left(\beta_{0} \ldots B_{k}\right)\right)=\bigcup_{j=1}^{k} x_{j}$. We define the homomorphism strip by, $\operatorname{strip}\left(A_{q}\right)=A$, for all $A_{q}$ in $V^{\prime}$.

If this claim is true then

since

$$
\alpha_{m}^{\prime}=\beta_{0} D_{1} \beta_{1} \ldots D_{k} \beta_{k} \Rightarrow x \text { in } G^{\prime}
$$

$$
\alpha_{m}^{\prime}=D_{1} \ldots D_{k} \Rightarrow x \text { in } F^{\prime} \text { and } \beta_{0} \cdots \beta_{k} \Rightarrow \lambda \text { in } G^{\prime} \text {, by definition. }
$$


We prove this by induction on $i$. It is trivially true for $i=1$, hence assume $i t$ has been established for $\alpha_{i}$ and $\alpha_{i}^{1}$. Consider the derivation step

$$
\alpha_{i} \Rightarrow \alpha_{i+1} \text { in } F^{\prime} \text {. }
$$

Note first that only productions of types $1^{\prime}, 2^{\prime}, 2^{\prime \prime}$ and $2^{\prime \prime}$ appear in $\left(^{*}\right)$. For types $1^{\prime}$ and $2^{\prime}$ the claim is surely true since, for example, using $D_{j}+E_{j} F_{j}$ in $\mu\left(\left[A_{j}, X_{j}\right] \rightarrow\left[B_{j}, Y_{j}\right]\left[C_{j}, Y_{j}\right]\right)$ we may use exactly the same production in $G^{\prime}$ and the change from $X_{j}$ to $Y_{j}$ can be carried out by the subscripted nonterminals.

For a type 2" production (and similarly for a type 2" production) $q: D_{j} \rightarrow E_{j}$ in $\mu\left(\left[A_{j}, X_{j}\right] \rightarrow\left[B_{j}, Y_{j} \cup\left\{C_{j}\right\}\right]\right)$, the production $D_{j} \rightarrow E_{j} C_{q}$ in $G^{\prime}$ does indeed ensure that an additional subscripted nonterminal has been introduced. This also ensures that sufficient nonterminals occur in $\beta_{0} \cdots \beta_{k}$ for the simulation of types $1^{\prime}$ and $2^{\prime}$ productions. Therefore we can construct $\alpha_{j+1}^{j}$ such that

$$
\alpha_{i}^{\prime} \Rightarrow \alpha_{i+1}^{i}
$$

and moreover $\alpha_{i+1}$ and $\alpha_{j+1}^{1}$ satisfy the claim.

This completes the proof of $L\left(F^{\prime}, \Rightarrow\right)=L\left(G^{*}, \Rightarrow\right)$ and also of the theorem.

We now turn to the 2 -table normal form theorem.

\section{Theorem 2.10}

Let $G=\left(V, \Sigma, P_{T}, \ldots, P_{n}, S\right)$ be an $n$-tabled ETOL form, where $n \geq 1$.

Then a 2-tabled ETOL form $F=\left(U, \Sigma, Q_{1}, Q_{2}, S\right)$ can be constructed such that $\mathcal{L}_{S}(F, \Rightarrow)=\mathcal{Z}_{S}(G, \Rightarrow)$.

Proof: Let $U=V \cup\{[X, 1]: X$ is in $V$ and $1 \leq i \leq n\}$,

$$
\begin{aligned}
Q_{1}= & \{X \rightarrow[X, 1]: X \text { is in } V\} \\
& \cup\{[X, i] \rightarrow[X, i+1]: 1 \leq i<n \text { and } X \text { is in } V\} \\
& \cup\{[X, n] \rightarrow N: X \text { is in } V\} \\
& \cup\{N \rightarrow N\},
\end{aligned}
$$

where $N$ is the universal blocking symbol, and

$$
\begin{aligned}
Q_{2}= & \{X \rightarrow N: X \text { is in } V\} \\
& \cup\{N \rightarrow N\} \\
& \cup\left\{[X, i] \rightarrow \alpha: X \rightarrow \alpha \text { is in } P_{i}, X \text { is in } V, 1 \leq i \leq n\right\} .
\end{aligned}
$$

Note that if $X \rightarrow \alpha$ is in $P_{i}$, then

$$
X \Rightarrow[X, 1] \Rightarrow \ldots \Rightarrow[X, i] \text { in } Q_{1}
$$

and $[X, i] \Rightarrow \alpha$ in $Q_{2}$. 
Conversely if $X \Rightarrow[X, 1] \Rightarrow \ldots \Rightarrow[X, i] \Rightarrow \alpha$ in $F$

then $X \rightarrow \alpha$ is in $P_{i}$.

Thus $L(G, \Rightarrow)=L(F, \Rightarrow)$.

Now productions in $G$ are "expansion-simulated" in $F$ and

derivations $X \Rightarrow \alpha_{1} \Rightarrow \ldots \Rightarrow \alpha_{i} \Rightarrow \alpha$ in $F, \alpha_{j}$ in $(U-V)^{+}, 1 \leq j \leq i, X$ in $V$ and $\alpha$ in $\forall *$ are "contraction-simulated" in $G$. However the simulation results proved earlier are too restrictive to be applied to the present situation, thus form equivalence has to be proved directly.

(i) $\mathcal{L}_{s}(G, \Rightarrow) \subseteq \mathcal{Z}_{s}(F, \Rightarrow)$.

This is straightforward, since for an arbitrary $G^{\prime} \Delta_{S} G(\mu)$ the construction given above can be carried out to given a $F^{\prime}$ which is obviously an interpretation of $F$. Moreover by the preceding remarks $L\left(G^{\prime}, \Rightarrow\right)=L\left(F^{\prime}, \Rightarrow\right)$.

(ii) $\mathcal{L}_{s}(F, \Rightarrow) \subseteq \mathcal{L}_{s}(G, \Rightarrow)$.

Consider an arbitrary interpretation $F^{\prime}=\left(U^{\prime}, \Sigma^{\prime}, Q_{1}^{\prime}, Q_{2}^{\prime}, S^{\prime}\right) \underset{s}{\Delta} F(\mu)$. We construct a $G^{\prime}=\left(V^{\prime}, \Sigma^{\prime}, P_{j}^{\prime}, \ldots, P_{n}^{\prime}, S^{\prime}\right) \Delta_{S} G\left(\mu^{\prime}\right)$ such that $L\left(G^{\prime}, \Rightarrow\right)=L\left(F^{\prime} \Rightarrow\right)$.

Let $V^{\prime}=\mu(V) \cap U$ ! and for a $11 ;, 1: \leq i \leq n$, define $P_{j}^{\prime}$ by:

$P_{j}^{\prime}=\left\{X^{\prime} \rightarrow \alpha^{\prime}: X^{\prime}\right.$ is in $V^{\prime}, \alpha^{\prime}$ is in $V^{\prime *}, X^{\prime} \Rightarrow^{+} Y^{\prime}$ in $Q_{1}^{\prime}$

and $Y^{\prime} \rightarrow \alpha^{\prime}$ is in $Q_{2}^{\prime}$ \}.

Now since $F^{\prime} \Delta F(\mu)$, for all $X^{\prime} \rightarrow \alpha^{\prime}$ in $P_{i}^{\prime}$, for all $i, 1 \leq i \leq n$ we have:

$$
\begin{aligned}
& \mu^{-1}\left(X^{\prime}\right) \Rightarrow \mu^{-1}\left(\beta_{1}\right) \Rightarrow \ldots \Rightarrow \mu^{-1}\left(\beta_{j}\right) \text { in } Q_{1} \text {, for some } j, \\
& 1 . \leq j \leq n \text { and } \mu^{-1}\left(\beta_{j}\right) \Rightarrow \mu^{-1}\left(\alpha^{1}\right) \text { in } Q_{2} .
\end{aligned}
$$

Immediately, since. $\mu^{-1}\left(\beta_{k}\right)$ is in $U-V$, we have $\beta_{k}$ is in $U^{\prime}-V^{\prime}$ and hence $\beta_{k}$ is not in $V^{\prime}$. Hence $L\left(F^{\prime}, \Rightarrow\right)=L(G, \Rightarrow)$.

It remains to demonstrate that $G^{\prime} \underset{s}{\Delta} G\left(\mu^{\prime}\right)$. Let $\mu^{\prime}(X)=\mu(X)$, for all $X$ in $V$. Clearly all conditions are trivially fulfilled except for $P_{j}^{\prime} \subseteq \mu\left(P_{j}\right)$. However from the above: $X^{\prime} \rightarrow \alpha^{\prime}$ is in $P_{j}^{\prime}$ iff $\left(^{*}\right)$ holds, but this in turn holds iff $\mu^{-1}\left(X^{\prime}\right) \rightarrow \mu^{-1}\left(\alpha^{\prime}\right)$ is in $P_{j}$, hence the result. 


\section{3 Completeness}

We have already mentioned EOL-completeness In Section 1, however, it is appropriate to consider a more general notion in this and the following sections.

Given a language family $\mathcal{X}$ we say that an ETOL form $F$ is $\mathcal{Z}$-complete if $\mathcal{Z}_{\mathrm{s}}(\mathrm{F}, \Rightarrow)=\mathcal{L}$. Thus, for example, $\mathrm{G}_{1}$ defined by the two tables

$$
\{S \rightarrow a ; S \rightarrow S ; S \rightarrow S S ; a \rightarrow S\}
$$

and

$$
\{\mathrm{S} \rightarrow \mathrm{S} ; \mathrm{a} \rightarrow \mathrm{S}\}
$$

is in fact ETOL-complete from the constructions of Theorem 2.4 and 2.10 . And, as we have seen previously, $G_{2}$ defined by:

$$
\{S \rightarrow a ; S \rightarrow S ; S \rightarrow S S ; a \rightarrow S\}
$$

is EOL-complete.

on the other hand $G_{3}$ defined by:

$$
\{S \rightarrow a ; S \rightarrow a S ; a \rightarrow a\}
$$

will be shown to be regular-complete, while $G_{4}$ defined by the two tables

$$
\begin{aligned}
& \{S \rightarrow S ; S \rightarrow S S ; a \rightarrow a\} \\
& \{S \rightarrow a ; a \rightarrow a\}
\end{aligned}
$$

will be shown to be EOL-complete. This particular result is af interest since

neither $\{S \rightarrow S ; S \rightarrow S S ; a \rightarrow a\}$

nor $\{S \rightarrow a ; a \rightarrow a\}$

are EOL-complete when taken alone. In fact, the first table gives the empty language and the second only the symbol sets.

An example of a synchro-EOL form which is EOL-complete is given by $G_{5}$ :

$$
S \rightarrow a ; S \rightarrow S ; S \rightarrow S S \text {. }
$$

Most of the results in this area are for propagating EOL forms, that is EPOL forms, hence in sections 3.1 and 3.2 we will consider EPOL forms and synchro-EPOL forms. This should serve to make the reader aware of some of the difficulties involved in this question. In section 3.3 we will briefly consider the completeness of ETOL forms, while in section 3.4 we will briefly examine regular-completeness.

\section{3.1 Two-symbol EPOL Forms}

A two-symbol EOL form is an EOL form whose oniy terminal is "a" and whose only nonterminal is "S". Similarly a two-symbol synchroEOL form is a synchro-EOL form whose only terminal is "a" and whose 
only nonterminals are "S" and "N", the universal blocking symbol. We will mainiy deal with the case when such a form is propagating, that is, an EPOL form.

The following two preliminary results are needed.

\section{Lemma 3.1}

Let $F$ and $G$ be two EOL forms (synchro-EOL forms), such that $F$ is EOL-complete and $G$ expansion simulates $F$. Then $G$ is EOL-complete.

Proof: By Lemma $1.10 \mathcal{Z}_{s}\left(F, \Rightarrow \subseteq \mathcal{Z}_{s}(G, \Rightarrow)\right.$, giving the result immediately.

Secondly, we have:

Lemma 3.2

Let $F=(\{S, a\},\{a\}, P, S)$ be a two-symbol EPOL form. If $F$ is E0L-complete, then

(i) $S \rightarrow$ a is in $P$,

(ii) P contains a production a $\rightarrow \alpha$ where $\alpha$ contains at least one occurrence of $S$,

(iii) $L(F, \Rightarrow)=a^{+}$,

(iv) $F$ is expansive, and

(v) $F$ is looping.

Proof: (i) Since $F$ is complete there must be a shortest derivation $S=\alpha_{0} \Rightarrow \alpha_{1} \Rightarrow \ldots \Rightarrow \alpha_{m}=a$ in $F$.

Furthermore $\left|\alpha_{i}\right|=1$ since $F$ is propagating and therefore $\alpha_{i}=S, 0 \leq i<m$. Hence $S \rightarrow$ a is in $P$.

(ii) Assume that each production for a is of the type $a \rightarrow a^{k} ; k \geq 1$.

We argue by contradiction that this cannot in fact be the case. Let $\ell=\operatorname{maxr}(F)$, choose a prime number $p$ so that $p>\ell^{3}$ and let

$L=\left\{a^{p}: i \geq 1\right\}$.

Since $F$ is complete there exists an $F^{\prime}=\left(V^{\prime}, \Sigma^{\prime}, P^{\prime}, S^{\prime}\right) \underset{s}{\Delta} F$ with $L\left(F^{\prime} \Rightarrow\right)=L$, that is, a is in $\Sigma^{\prime}$. Since $a \rightarrow x$ in $P^{\prime}$ implies $x$ is in $\Sigma^{\prime *}$, by assumption, then $x=a^{m}$ for some $m \geq 0$, otherwise $L\left(F^{\prime}, \Rightarrow\right)$ would contain words not in $L$. If $m>1$ then $a^{p} \Rightarrow a^{p m}$ with $a^{p m}$ in L. However $1<m<p$

by assumption, hence $p<p m<p^{2}$, a contradiction. Thus either $m=0$ or $m=1$. Since $F$ is propagating $m=0$ is ruled out. If $m=1$, 
then $L\left(F^{\prime} \Rightarrow\right)$ is context-free, a contradiction.

Hence we have demonstrated that there must be a production $a \rightarrow \alpha$ with $|\alpha|_{S} \geq 1$.

(iii) Since $a^{+}$is an EOL language, then there must exist $F^{\prime} \underset{s}{4} F$ with $L\left(F^{\prime} \Rightarrow\right)=a^{+}$. By Lemma 1.3 this implies $a^{+} \subseteq L(F, \Rightarrow)$ and hence $L(F, \Rightarrow)=a^{+}$.

(iv) By ( $i$ i) there is a production $a \rightarrow a_{1} S \alpha_{2}$ in $P$ and by ( $\left.i i i\right)$ there is a derivation $S \Rightarrow$ a ${ }^{+}$in $F$. Hence there is a derivation $S \Rightarrow a a \Rightarrow \alpha_{1} S \alpha_{2} \alpha_{1} S \alpha_{2}$ in $F$ and therefore $F$ is expansive.

(v) This result is proved indirectly by using the properties of the corresponding EDTOL grammar. In the following Lemma it is proved that for an EOL grammar $F^{\prime} 4 F$ with $L\left(F^{\prime}, \Rightarrow\right)=\left\{a^{i} b^{i} c^{i}: i \geq 1\right\}$ the corresponding EDTOL grammar $G$ has $L(G, \Rightarrow)=L\left(F^{\prime}, \Rightarrow\right)$.

Immediately $G$ must be looping, therefore $F^{\prime}$ must be looping and hence $F$ is looping.

Let $H=(V, \Sigma, P, S)$ be an EOL grammar where $V=\left\{X_{1}, \ldots, X_{m}\right\}$ say, and the productions for $x_{i}$ are denoted:

$$
x_{i} \rightarrow \alpha_{i, 1}, \ldots, x_{i} \rightarrow \alpha_{i, n_{i}} \text {. }
$$

Then the corresponding EDTOL grammar is defined by

$$
\begin{aligned}
G=(V, \Sigma, P & (1, \ldots, 1), P(1,1, \ldots, 2), \ldots, P\left(k_{1}, \ldots, k_{m}\right), \ldots, \\
& P_{\left.\left(n_{1}, \ldots, n_{m}\right), s\right) \text { where }} \\
& P_{\left(k_{1}, \ldots, k_{m}\right)}=\left\{x_{1} \rightarrow \alpha_{1, k_{1}}, \ldots, x_{m} \rightarrow \alpha_{n, k_{m}}\right\}
\end{aligned}
$$

for $1 \leq k_{j} \leq n_{j}, \quad l \leq j \leq m$.

That is G contains a deterministic set of productions for each combination of productions for $X_{1}, \ldots, X_{m}$ from $P$. Note that $G$ is propagating if $H$ is propagating.

\section{Lemma 3.3}

Let $H$ and $G$ be as above and assume $L(H, \Rightarrow)=\left\{a^{i} b^{i} c^{i}: i \geq 1\right\}=L$, say.

$$
\text { Then } L(G, \Rightarrow)=L \text {. }
$$

Proof: It is clear that $L(G, \Rightarrow) \subseteq L$. Conversely, consider any $x$ in $L$ and a derivation tree for $x$. Consider any level $j$ of the derivation tree. Clearly if $x$ is derived in one step in $H$, then it is also trivially in $L(G, \Rightarrow)$. Hence we may assume the derivation tree for $x$ has at least 3 levels. Secondly we may assume there is at least one level $j$, which contains two appearances of some symbol. If this is 
not so then the given derivation tree is also a derivation tree of $G$.

Let the symbol which occurs at least twice on level $j$ be $x$.

Now $x \Rightarrow x_{1}$ and $x \Rightarrow^{+} x_{2}$, where $x=u x_{1} u x_{2} w$. Immediately

$\left\{u x_{1} v x_{2} w, u x_{1} v x_{1} w, u x_{2} v x_{2} w, u x_{2} v x_{1} w\right\} \subseteq L$, which implies $x_{1}=x_{2}$. Hence we may assume the same production is applied to $x$ at level $j$. or, in other words a single table of G suffices at level $j$, giving the result.

If $F$ is a two-symbol synchro-EOL form then we can also obtain a result corresponding to Lemma 3.2 namely,

\section{Lemma 3.1}

Let $F=(\{S, a\},\{a\}, P, S)$ be a two-symbol synchro-EOL form. If $F$ is EOL-complete, then

(i) $S \rightarrow a$ is in $P$,

(ii) $L(F, \Rightarrow)=a^{+}$,

(iii) $F$ is expansive, and

(iv) $F$ is looping.

Proof: These may be proved analogously to Lemma 3.2 , noting that we can convert $F$ to a form-equivalent propagating form by Theorem 2.9 which is expansive iff $F$ is expansive.

We are now in a position to characterize EOL-completeness for two-symbol synchro-EPOL forms.

\section{Theorem 3.5}

A two-symbol synchro-EPOL form $H$ is EOL-complete iff it contains the production $S \rightarrow S$ and for some $i \geq 2$, all of the productions $S \rightarrow S^{i} ; S \rightarrow a, \ldots, S \rightarrow a^{i-1}$.

Proof: if: Clearly the form with productions $S \rightarrow S ; S \rightarrow S S$; $S \rightarrow$ a is EOL-complete. For an arbitrary value of $i>2$ completeness follows from the following claim.

Czaim: Let $F=(\{S, a\},\{a\}, P, S)$ be a two-symbol synchro-EPOL form with $P=\left\{S \rightarrow S ; S \rightarrow S^{i}\right\} \cup\left\{S \rightarrow a^{j}: 1 \leq j<i\right\}$ for some $i>2$. Then $F$ is EOL-complete. 
Proof of Claim: Every EOL language can be generated by a synchro-EPOL grammar which has productions of types (i) $A \rightarrow a,(i i) A \rightarrow B$ and (iji) $A \rightarrow B C$. Let $G=(V, \Sigma, Q, Z)$ be such a grammar. Construct a new EPOL grammar $F^{\prime}=\left(V^{\prime}, \Sigma, P^{\prime}, Z^{\prime}\right)$ as follows:

(1) for each word $x$ in $L(G, \Rightarrow)$ with $|x|<i$ take $Z^{\prime} \rightarrow x$ into $P^{\prime}$,

(2) for each production $A \rightarrow B$ in $Q$ take $A \rightarrow B$ into $P^{\prime}$,

(3) for each production $A \rightarrow B C$ in $Q$ take $A \rightarrow[B C]$ into $P^{\prime}$, where [BC] is a new nonterminal.

Now consider the new nonterminals $[\alpha], 2 \leq|\alpha|<i, \alpha$ in $(V-\Sigma)^{+}$. For each derivation $\alpha \Rightarrow \beta$ in $G$ with $2 \leq|\alpha|<i$ we have $2 \leq|\beta| \leq 2 i-2$. In the case $\beta$ is in $\Sigma^{+}$we have $2 \leq|B| \leq i-1$. We now "simulate" such derivations in $F^{\prime}$ by way of the new nonterminals as follows: For all $\alpha$ in $(V-\Sigma)^{+}, 2 \leq|\alpha|<i$, and derivations $\alpha \Rightarrow \beta$ in $G$.

(4) if $\beta$ is in $\Sigma^{+}$, take $[\alpha] \rightarrow \beta$ into $P^{\prime}$, and

(5) if $\beta$ is in $(V-\Sigma)^{+}$, then

(i) if $|\beta|<$ i take $[\alpha] \rightarrow[\beta]$ into $P^{\prime}$,

(ii) if $|\beta|=i$ take $[\alpha] \rightarrow \beta$ into $P^{\prime}$, and

(iii) if $|\beta|>$ i take $[\alpha] \rightarrow \beta_{1}\left[\beta_{2}\right]$ into $p^{\prime}$, where $\beta=\beta_{1} \beta_{2}$ and $\left|\beta_{1}\right|=i-2$.

Letting $V^{\prime}$ be defined appropriately, it should be clear that $F^{\prime} \vec{s} F$ and moreover that $L\left(F^{\prime}, \Rightarrow\right)=L(G, \Rightarrow)$. This complete the claim.

Returning to the theorem we have $F$ of the claim is an interpretation of $H$, hence $H$ is also EOL-complete.

only if: First observe that since $H$ is synchronized we may assume the productions of $H$ are either of the type $S \rightarrow S^{i}$ or of the type $S \rightarrow a^{j}$, mixtures of symbols on the right hand sides being irrelevant, since they cause blocking. By Lemma $3.4 \mathrm{H}$ contains $\mathrm{S} \rightarrow \mathrm{S}$ since $\mathrm{H}$ must be looping and $H$ contains $S \rightarrow S^{i}$ for some $i \geq 1$ since $H$ must be expansive. Choose the smallest such $i \geq 2$. Then $H$ must contain all of the productions

$$
S \rightarrow a, \ldots, S \rightarrow a^{i-1}
$$

because, otherwise $L(H, \Rightarrow) \neq a^{*}$.

This result can be extended to $\left\{S, a, \ldots, a_{m}\right\}-$ synchro-EPOL forms $H=\left(\left\{s, a_{1}, \ldots, a_{m}\right\},\left\{a_{\eta}, \ldots, a_{m}\right\}, P, s\right)$ where $m \geq 1$, as follows:

\section{Theorem 3.6}

If an $\left\{S, a_{1}, \ldots, a_{m}\right\}$-synchro-EPOL form $H$ is EOL-complete, then, for some $i$, the $\left\{S, a_{i}\right\}$-synchro-EPOL form $H^{\prime}$ obtained from $H$ by omitting 
all the productions involving terminals other than $a_{i}$, is also EOLcomplete.

Proof: Since $H$ is EOL-complete it contains the productions $S \rightarrow S$ and $S \rightarrow S^{j}$, for some $j \geq 2$.

Further since $H$ is EOL-complete there is an $H^{\prime \prime} \Delta H$ such that $L\left(H^{\prime \prime} \Rightarrow\right)=a^{+}$. This implies there is an $i, 1 \leq i^{5}$, such that $a_{i}^{+} \subseteq L(H, \Rightarrow)$. Now apply Theorem 3.5 to the $\left\{S, a_{i}\right\}$-form obtained with respect to this $i$.

We now turn to the study of two-symbol EPOL forms. Since this situation is much more difficult, we will restrict our attention even further to short two-symbol EP0L forms. In this case only the following productions may occur:

(1) $S \rightarrow a ; S \rightarrow a a ; S \rightarrow a S ; S \rightarrow S a ; S \rightarrow S ; S \rightarrow S S$;

(2) $a \rightarrow a ; a \rightarrow a a ; a \rightarrow a S ; a \rightarrow S a ; a \rightarrow S ; a \rightarrow S S$.

Let $H=(\{S, a\},\{a\}, P, S)$ be a short two-symbol EPOL Form. Four cases are distinguished according to the value of $D=P \cap\{S \rightarrow S ; a \rightarrow S\}$.

Case 1: $\quad D=\{S \rightarrow S ; a \rightarrow S\}$.

\section{Theorem 3.7}

$H$ is EOL-complete iff $P \cap\{S \rightarrow$ aS; $S \rightarrow S a ; S \rightarrow S S\} \neq \emptyset$.

Proof: Clearly $\{S \rightarrow a ; S \rightarrow S ; a \rightarrow S\} \subseteq P$. If $S \rightarrow S S$ is a $\rightarrow$ so in $P$, then $H$ is EOL-complete.

Assume $S \rightarrow$ aS is in $P$, then we have

$$
\begin{array}{ll}
S \Rightarrow S \Rightarrow a & S \Rightarrow a S \Rightarrow S S \\
S \Rightarrow S \Rightarrow S & a \Rightarrow S \Rightarrow S
\end{array}
$$

in $H$. Hence this is EOL-complete, as is the remaining case.

Conversely, assume $H$ is EOL-complete and $\mathrm{P} \cap \mathrm{S} \rightarrow \mathrm{S} \rightarrow \mathrm{S} \rightarrow \mathrm{Sa}$; $S \rightarrow S S\}=0$. Now $H$ is expansive by Lemma 3.2 , hence it contains at least one of the productions:

$S \rightarrow a a ; a \rightarrow a a ; a \rightarrow a S ; a \rightarrow S a ; a \rightarrow S S$.

Examining each possibility in turn shows that each infinite language in $\mathcal{L}_{s}\left(H, \Rightarrow\right.$ contains a word of length 1 or 2 . Thus $L=\left\{a^{i}: i \geq 3\right\}$ is not in $\mathcal{L}_{\mathrm{S}}(H, \Rightarrow)$, a contradiction. 
Hence we have shown that:

$$
\begin{aligned}
& H_{1}: S \rightarrow a ; S \rightarrow S ; S \rightarrow S S ; a \rightarrow S ; \\
& H_{2}: S \rightarrow a ; S \rightarrow S ; S \rightarrow S a ; a \rightarrow S ; \\
& H_{3}: S \rightarrow a ; S \rightarrow S ; S \rightarrow a S ; a \rightarrow S ;
\end{aligned}
$$

are EOL-complete.

$$
\begin{aligned}
& \quad \text { It is clear that } \\
& H_{4}: S \rightarrow a ; S \rightarrow S ; S \rightarrow S S ; a \rightarrow S S
\end{aligned}
$$

is also EOL-complete, since the only difference between $\mathrm{H}_{1}$ and $\mathrm{H}_{4}$ is the production $a \rightarrow S$ as against the production a $\rightarrow S S$. As these productions are used for blocking purposes, they do not hinder EOLcompleteness. In fact a $\rightarrow S$ may be replaced by either a $\rightarrow$ a $S$ or $a \rightarrow S a$ in $\mathrm{H}_{1}$ without affecting its completeness.

We will show that if $D=\emptyset$ then $H$ is incomplete, while for $D=\{a \rightarrow S\}$ there are complete and incomplete forms. We first deal with:

Case 2: $D=\{S+S\}$.

This means that $a \rightarrow S$ is not in $P$.

We will see that even here a complete classification cannot be carried out. We first have:

Theorem 3.8

$$
\text { If } S \rightarrow S S \text { is in } P \text {, then } H \text { is EOL-complete iff }
$$

$P \cap\{a \rightarrow a S ; a \rightarrow S a ; a \rightarrow S S\} \neq \emptyset$.

Proof: By the remarks above for $\mathrm{H}_{4}$ and from Lemma 3.2 .

Theorem 3.9

If $\{S \rightarrow S S ; a \rightarrow a ; a \rightarrow a a ; a \rightarrow S\} \cap P=\emptyset$ then $H$ is EOL-complete iff $\{a \rightarrow S S ; S \rightarrow a a\} \subseteq P$ and $P \cap\{S \rightarrow a S ; S \rightarrow S a\} \neq \emptyset$.

Proof: if: We have the following two-step derivations in $\mathrm{H}$ :

$$
\begin{aligned}
& S \Rightarrow S \Rightarrow S \\
& S \Rightarrow a S \Rightarrow S S S \text { (or } S \Rightarrow S a \Rightarrow S S S \text { ) } \\
& S \Rightarrow S \Rightarrow a \\
& S \Rightarrow S \Rightarrow a a
\end{aligned}
$$

which by Theorem 3.5 is EOL-complete, since $H$ can easily be synchronized. only if: Assume $H$ is complete and $P \cap\{S \rightarrow S a ; S \rightarrow a S\}=\emptyset$. Then, since $S \rightarrow S S, S \rightarrow S a$ and $S \rightarrow$ a $S$ are not in $P$ every infinite language in $\mathcal{L}_{\mathrm{s}}(H, \Rightarrow)$ contains a word of length 1 or 2 . Hence $H$ is incomplete, a contradiction. 
If $H$ is complete and $\mathrm{a} \rightarrow \mathrm{SS}$ is not in $\mathrm{P}$, then $\mathrm{S} \rightarrow \mathrm{SS}$, $\mathrm{a} \rightarrow \mathrm{SS}$, $a \rightarrow a, a \rightarrow a a, a \rightarrow S$ are not in $P$, in which case there is no nonterminal derivation $S \Rightarrow+$ aaa. Hence $\{a a\} \& \mathcal{L}_{S}(H, \Rightarrow)$ and therefore $H$ is incomplete.

Finally if $H$ is complete and $S$, aa is not in $P$, then aa is not in $L(H, \Rightarrow)$, a contradiction.

The remaining subcases have not, at the time of writing, been fully classified. We therefore pass on to cases 3 and 4 .

Caee 3: $\quad \mathrm{D}=\emptyset$.

Hence $P$ contains neither $S \rightarrow S$ nor $a \rightarrow S$.

Consider the following strengthening of part $(v)$ of Lemma 3.2 , which we state without proof.

\section{Proposition 3.10}

If an EPOL form $F=(V, \Sigma, P, S)$ is $E O L$-complete then $F$ must have nonterminal looping, that is, $A \Rightarrow A$ for some $A$ in $V-\Sigma$.

We merely remark that if $F$ does not have nonterminal looping then a language such as $\left\{a^{i} b^{i} c^{i} d^{i}: i \geq 1\right\}$ cannot be generated by any interpretation of $F$.

Immediately from this Proposition we obtain:

\section{Theorem 3.11}

If $P \cap\{S \rightarrow S ; a \rightarrow S\}=\emptyset$ then $H$ is not EOL-complete.

This leaves the final case.

Case 4: $\quad D=\{a \rightarrow S\}$.

This means that $P$ does not contain $S \rightarrow S$, and therefore interpretations of $H$ do not contain nonterminal chain productions, that is, productions of the type $A \rightarrow B$, where both $A$ and $B$ are nonterminals. We now consider the subcase

Case 4.1: $\quad\{a \rightarrow S ; a \rightarrow a ; S \rightarrow a\} \subseteq P$, which we are able to treat completely. The following result we state without proof. 
Proposition 3.12

Every EPOL language $L$ can be generated by a weakly synchronized, separated and chain-free EPOL grammar:

We say an EPOL grammar $F=(V, \Sigma, P, S)$ is weakly synchronized if for all $x$ in $L(F, \Rightarrow), x \Rightarrow^{+}$a implies $\alpha$ is not in $\Sigma^{*}$.

An EOL grammar $F=(V, \Sigma, P, S)$ is chain-free if each derivation tree in $F$ whose frontier is terminal is chain-free. Such a derivation tree is said to be chain-free if it contains at least one root-tofrontier path $u_{0}, \ldots, u_{n}$ in which each node $u_{i}$ has at least two successors $0 \leq i \leq n-2$.

We now make use of proposition 3.12 to obtain a particular normal form which is useful in the context of completeness. We first have:

\section{Lemma 3.13}

Every EPOL Tanguage $L$ can be generated by an EPOL grammar which contains no nonterminal chain productions.

Proof: Let $G=(V, \Sigma, P, S)$ be an EPOL grammar such that $L(G, \Rightarrow)=L$ and $G$ fulfills the conditions of Proposition 3.12 .

Without loss of generality we can assume there is a partition of $V$ and $P$ such that $V=V_{1} \cup V_{2} \cup \Sigma, s$ is in $V_{2}$, $P=P_{1} \cup P_{2}, P_{1} \subseteq\left(V_{1} \cup \Sigma\right)^{\prime} \times\left(\Sigma \cup V_{1}^{+}\right)$and $P_{2} \leq V_{2} \times\left(\Sigma \cup V_{1}^{*} V_{2} V_{1}^{+} \cup V_{1}^{+} V_{2} V\left(\frac{*}{1}\right)\right.$. Essentially $V_{2}$ symbols are used to keep track of a chain-free path in a chain-free derivation.

We now use this partition to construct a new EPOL grammar in which each derivation tree is almost twice as high as its counterpart in $G$.

Let $\mu$ be a homomorphism defined on $V *$ by $\mu(A)=A$ for $A$ in $V_{1} \cup \Sigma$ and $\mu(A)=\bar{A}$ for $A$ in $V_{2}$ and let $V_{3}=\{\vec{A}: A$ in $V-\Sigma\}$.

Construct $F=\left(\left\{V \cup V_{3} \cup\{N\}, \Sigma \cup V_{3}, Q, S\right)\right.$, where $N$ is a new nonterminal, as follows:

$$
\begin{aligned}
Q= & \left\{A \rightarrow \mu(\alpha): A \text { is in } V_{2}, A \rightarrow \alpha \text { is in } P_{2}\right\} \\
& u\left\{\bar{A} \rightarrow \alpha: A \text { is in } V_{1}, A \rightarrow \alpha \text { is in } P_{1}\right\} \\
& u\left\{A \rightarrow A: A \text { is in } V_{1}\right\} \\
& u\left\{\bar{A} \rightarrow A: A \text { is in } V_{2}\right\} \\
& u\{a \rightarrow N ; \text { a is in } \Sigma\} \\
& u\{N \rightarrow N N\} .
\end{aligned}
$$

Since each G-derivation-tree is chain-free this implies there is a member of $v_{2}$ appearing on each level with at least one member of $v_{1}$. 
On the other hand in $F$ on each level other than the frontier either the $v_{2}$ symbol is marked or the $v_{1}$ symbols are marked, but not both. Hence the new terminals $v_{3}$ never occur alone on any level. Moreover each level in the G-derivation tree becomes two levels in the corresponding $F$-derivation tree apart from the frontier. For example, we might have:
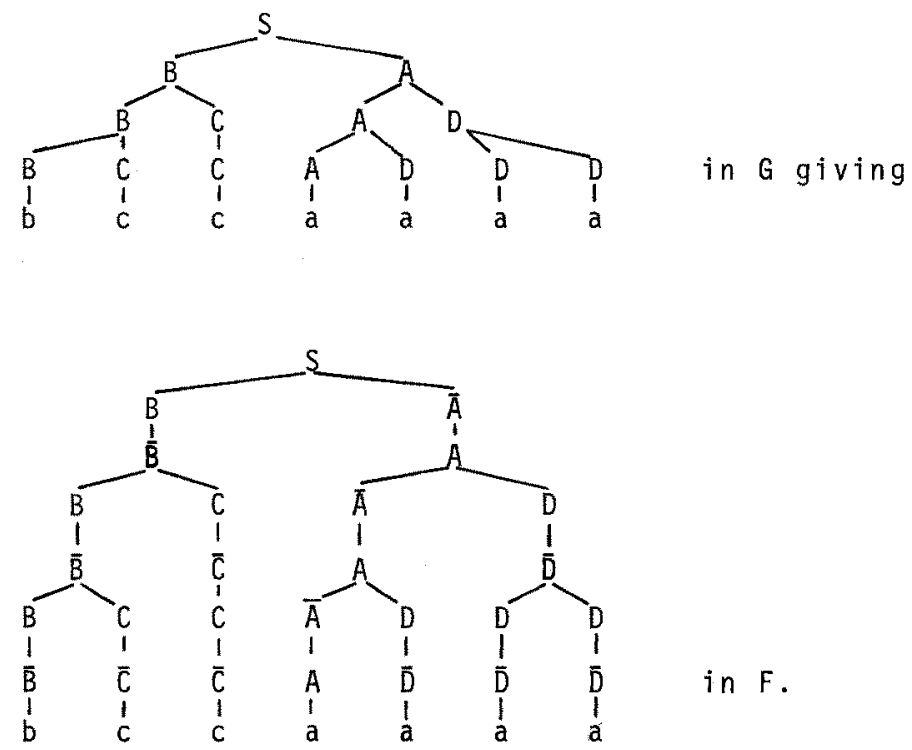

Clearly $L(G, \Rightarrow) \subseteq L(F, \Rightarrow)$. The reverse inclusion and hence equality is obtained by the observations above which imply that the only possible way for an F-derivation to terminate is if it is one of the types: either $S \Rightarrow^{2 i} \alpha \Rightarrow x$ in $\Sigma^{+}$, where $i>0, \alpha$ is in $V_{3}^{*} V_{2} V_{3}^{*}$ and $|\alpha| \geq 2$ or $\quad s \Rightarrow x$ in $\Sigma^{+}$.

By the construction nonterminal chain productions $A \rightarrow B$ have become derivations of the type $A \Rightarrow \bar{A} \Rightarrow B$. This concludes the Lemma.

We now consider how to obtain a short EPOL grammar with no nonterminal chain productions.

Theorem 3.14

Every EPOL language $L$ can be generated by a short EPOL grammar which contains no nonterminal chain productions. 
Proof: We will provide a sketch of the proof, leaving the details to be filled in by the reader. As in Theorem 3.13 without loss of generality let $G=(V, \Sigma, P, S)$ be an EPOL grammar with $L(G, \Rightarrow)=t$, fulfilling not only the conditions of Proposition 3.12 but also $V=V_{1} \cup V_{2} \cup \Sigma, P=P_{1} \cup P_{2}, S$ is in $V_{2}, P_{1} \subseteq\left(V_{1} \cup \Sigma\right) \times\left(\Sigma \cup V_{1}^{+}\right)$ and $P_{2} \subseteq V_{2} \times\left(\Sigma u V_{1}^{*} V_{2} V_{1}^{+} u v_{1}^{+} v_{2} V_{1}^{*}\right)$.

We further assume that $S$ does not appear on the right side of any production in $P$.

Now applying Lemma 2.3 to $G$ gives rise to an equivalent EPOL grammar $G^{\prime}-\left(V^{\prime}, \Sigma, P^{\prime}, S\right)$ which has the property that $G^{\prime}$ is short. The construction has the effect of simulating productions in $G$ by derivations in $G^{\prime}$ of length $m-1$, where $m=\max (G)$. Furthermore for each $X \rightarrow \alpha$ in $P$ the derivation $X \Rightarrow^{m-1} \alpha$ in $G^{\prime}$ is unique. Now without loss of generality we may assume $m$ is odd and that in the simulating derivation

$$
x \Rightarrow \alpha_{1} \Rightarrow \ldots \Rightarrow \alpha_{m-2} \Rightarrow \alpha
$$

in $G^{\prime}$ of $X \rightarrow \alpha$ in $P$, each $\alpha_{i}, 1 \leq i \leq m-2$, consists of unique new symbols.

Note that if $m$ is not odd it is straightforward to add a

further step in the shortening construction giving simulating derivations of length $m$, rather than $\mathrm{m}-1$.

clearly many chain productions have been introduced. We now ensure these are not nonterminal chain productions by constructing $F=(U, \Delta, Q, S)$ as follows.

First, take al1 S-productions in $P$ which are short into $Q$. For each of the remaining $s$-productions $s \rightarrow x_{1} \ldots x_{r}, r>2$, add sufficient productions to $Q$ to simulate them:

$$
\begin{aligned}
& s \rightarrow x_{1}^{1}\left[x_{2} \ldots x_{r}\right] ; x_{1}^{1} \rightarrow x_{1}^{2} ;\left[x_{2} \ldots x_{r}\right] \rightarrow x_{2}^{2}\left[x_{3} \ldots x_{r}\right] ; \\
& x_{1}^{2} \rightarrow x_{1}^{3} ; x_{2}^{2} \rightarrow x_{2}^{3} ;\left[x_{3} \ldots x_{r}\right] \rightarrow x_{3}^{3}\left[x_{4} \ldots x_{r}\right] \\
& \ldots \ldots \\
& x_{1}^{r-2} \rightarrow x_{1} ; \ldots, x_{r-2}^{r-2} \rightarrow x_{r-2} ;\left[x_{r-1} x_{r}\right] \rightarrow x_{r-1} x_{r}
\end{aligned}
$$

where the symbols $\left[x_{i} \ldots x_{r}\right]$ are nonterminals and the symbols $x_{j}^{i}$ are terminals.

Second, for each nonterminal $A$ in $V_{2}-\{S\}$, the "chain free" nonterminals, and for each step of the simulating derivation

$$
A \Rightarrow \alpha_{1} \Rightarrow \ldots \Rightarrow \alpha_{m-2} \Rightarrow \alpha=\beta_{1} B \beta_{2}, B \text { in } V_{2}
$$

of a production $A \rightarrow \alpha$ in $P_{2}, \alpha$ not in $\Sigma$, let $\alpha_{i}$ be terminal, $i=1,3, \ldots, m-2, \alpha_{i}$ be nonterminal, $i=2,4, \ldots, m-3$ and $\beta_{1} \beta_{2}$ be terminal. similarly for each nonterminal $A$ in $V_{1}$, and for each step of the simulating derivation 


$$
A \Rightarrow \alpha_{1} \Rightarrow \ldots \Rightarrow \alpha_{m-2} \Rightarrow \alpha_{m-1}=\alpha
$$

of a production $A \rightarrow \alpha$ in $P_{1}$, $\alpha$ not in $\Sigma$, let $A$ be terminal, $\alpha_{j}$ be terminal, $i=2,4, \ldots, m-1$ and $\alpha_{i}$ be nonterminal, $i=1,3, \ldots, m-2$.

Add these productions to $Q$.

Finally take the terminating productions in $P$ into $Q$ and the blocking productions $\mathrm{a} \rightarrow \mathrm{N}$ and $\mathrm{N} \rightarrow \mathrm{Na}$.

As in Theorem 3.13 we have removed nonterminal chain productions by changing them into non-nonterminal chain productions. In simulating an $S$-production $S \rightarrow \alpha B B, B$ in $V_{2}$, the derivation is a nonterminal one, and from thereon in the $V_{2}$-nonterminal gives rise to "pseudoterminals" at every odd step while a $V_{7}$-nonterminal gives rise to "pseudo-terminals" at every even step, as in Theorem 3.13. Finally, a terminal word is only produced when the previous step of the derivation consists of one $V_{2}$-nonterminal and $V_{1}$-pseudo-terminals. At the following step blocking occurs. Hence $L(F, \Rightarrow)=L(G, \Rightarrow)=L$.

We now obtain:

\section{Corozzary 3.15}

$\mathrm{F}_{1}: S \rightarrow \mathrm{a} ; \mathrm{S} \rightarrow \mathrm{a} a ; \mathrm{S} \rightarrow \mathrm{aS} ; \mathrm{S} \rightarrow \mathrm{Sa} ; \mathrm{a} \rightarrow \mathrm{a} ; \mathrm{a} \rightarrow \mathrm{S} ; \mathrm{a} \rightarrow \mathrm{SS}$ is EOL complete.

In fact by taking greater care in the specification of terminals and nonterminals in the previous theorem, the production $S \rightarrow$ a a can be dropped. In particular, for $X \rightarrow Y Z$ in $Q$ let both $Y$ and $Z$ be nonterminal if $X$ is terminal and only $Z$ be nonterminal otherwise.

Let $G_{1}$ be defined by the productions:

$$
\begin{aligned}
& Z \rightarrow b ; Z \rightarrow b b ; Z \rightarrow a S ; Z \rightarrow S a ; \\
& S \rightarrow a ; S \rightarrow b ; S \rightarrow a S ; S \rightarrow S a ; \\
& a \rightarrow S ; a \rightarrow S S ; a \rightarrow b ; b \rightarrow N ; \\
& N \rightarrow N b ;
\end{aligned}
$$

where $Z$ is the sentence symbol. Then "a" is a "pseudo-terminal" and "b" the real terminal. Clearly $G_{1} \underset{S}{\Delta} F_{1}$ and moreover $G_{1}$ is EOL-complete since $F$ of Theorem 3.14 is an interpretation of $G_{1}$.

Returning to our main theme, namely demonstrating that $H$ is EOL-complete, we have:

\section{Lemma 3.16}

An $E O L$ form $F=(\{S, a\},\{a\}, P, S)$ with $\{S \rightarrow a ; a \rightarrow a\} \leq P$ is EOL-complete if conditions (c1) - (c3) are met.

(c) $S \Rightarrow^{*}$ a; $S \Rightarrow^{*}$ aa; $S \Rightarrow^{*}$ aS; $S \Rightarrow^{*}$ Sa via nonterminal derivations in F. 
(c2) There is a natural number $k$ and there are $k$-step derivations in $\mathrm{F}$

$S \Rightarrow a ; S \Rightarrow^{k}$ aS; $S \Rightarrow^{k} S a ; a \Rightarrow^{k} S ; a \Rightarrow^{k} S S$

such that any combination of two of these derivations such that one begins with a and the other with $S$ has at least one $S$ at each intermediate step.

(c3) Fallows "blocking" of terminals, that is, there is a F-derivation a $\Rightarrow \alpha_{1} \Rightarrow \ldots \Rightarrow \alpha_{i} \Rightarrow \ldots$

such that each $\alpha_{j}, i \geq 1$ contains at least one $S$.

Proof: Again we only provide a proof sketch. Since $G_{1}$ is EOL-complete it suffices to prove that $\mathcal{Z}_{s}\left(G_{1}\right) \subseteq \mathcal{Z}_{s}(F)$.

To this end consider an arbitrary $G_{j} \underset{S}{\Delta} G_{1}$. We sketch how a simulating interpretation $F^{\prime}$ of $F$ can be constructed. For each word $x$ in $L\left(G_{j}, \Rightarrow\right.$ with $|x| \leq 2$ a unique isolating interpretation of the nonterminal derivations $S \Rightarrow^{*}$ a or $S \Rightarrow^{*}$ a a available by (cl) can be constructed.

For each word $x$ in $L\left(G_{j}^{\prime} \Rightarrow\right)$ of length greater than 2 a $G_{1}^{\prime}-$ derivation for $x$ :

$$
Z^{\prime}=\alpha_{0}^{+} \Rightarrow \alpha_{1}^{1} \Rightarrow \ldots \Rightarrow \alpha_{m}^{\prime}=x, m \geq 2
$$

is the image of a $G_{1}$-derivation

$$
Z \Rightarrow \alpha_{1} \Rightarrow \ldots \alpha_{m} \text {, }
$$

where $\alpha_{1}$ is either aS or Sa, $\alpha_{j}$ is in $\{S, a\}^{+}, 1 \leq i<m$ and $\alpha_{m}$ is in $\{b\}^{+}$.

Now $\alpha_{0}^{\prime} \Rightarrow \alpha_{j}^{\prime}$ can be isolated via interpretations of $S \Rightarrow{ }^{*}$ as or $S \Rightarrow$ Sa available in (cl) as nonterminal derivations. Since only interpretations of $S \rightarrow a ; S \rightarrow a S ; S \rightarrow S a ; a \rightarrow S ; a \rightarrow S S$ are applied to $\alpha_{i}, 1 \leq i<m-1$, and there is always at least one image of "a" and one image of " $S$ " in each $\alpha_{i}^{1}, 1 \leq i<m$, then the (c2) k-step derivations of $F$ can be used via isolation to simulate as a nonterminal derivation $\alpha_{j}^{\prime} \Rightarrow \alpha_{i+1}^{+}$in $F^{\prime}$ the derivation step $\alpha_{i}^{j} \Rightarrow \alpha_{i+1}^{i}$ in $G_{1}^{\prime}$. The step $\alpha_{m-1}^{\prime} \Rightarrow \alpha_{m}^{\prime}$ can be obtained directly since $S \rightarrow$ and $a \rightarrow$ a are in $P$.

Finally, "blocking" in Gi can be simulated in $F^{\prime}$ because of (c3). Hence $F$ is EOL-complete.

We now have:

Theorem 3.17

The EPOL forms

$S \rightarrow a ; S \rightarrow a S ; a \rightarrow a ; a \rightarrow S$

and $S \rightarrow a ; S \rightarrow S a ; a \rightarrow a ; a \rightarrow S$ are EOL-complete. 
Proof: We only show the completeness of one, the other follows by symmetry. We show that

$$
S \rightarrow a ; S \rightarrow a S ; a \rightarrow a ; a \rightarrow S
$$

fulfills conditions (c1) - (c3) of Lemma 3.16.

clearly (cl) is met since

$$
\begin{aligned}
& S \Rightarrow a ; S \Rightarrow a S \Rightarrow a a ; S \Rightarrow a S ; \\
& S \Rightarrow a S \Rightarrow S a
\end{aligned}
$$

and (c3) is met since we may have:

$$
\mathrm{a} \Rightarrow \mathrm{S} \Rightarrow \mathrm{aS} \Rightarrow \mathrm{Sa} \Rightarrow \mathrm{aS} \Rightarrow \ldots \text {. }
$$

To see that (c2) is met consider the following derivations:

$$
\begin{aligned}
& S \Rightarrow a \Rightarrow S \Rightarrow a \Rightarrow S \Rightarrow a \\
& S \Rightarrow a S \Rightarrow S a \Rightarrow a S \Rightarrow S a \Rightarrow a S \\
& S \Rightarrow a S \Rightarrow S a \Rightarrow a a \Rightarrow a S \Rightarrow S a \\
& a \Rightarrow S \Rightarrow a \Rightarrow S \Rightarrow a \Rightarrow S \\
& a \Rightarrow S \Rightarrow a S \Rightarrow S a \Rightarrow a a \Rightarrow S S
\end{aligned}
$$

Note that for each step of an S-derivation which is completely terminal the a-derivations have a nonterminal at the corresponding step and vice versa. Hence the result.

It is clear that the EPOL forms of Theorem 3.17 are minimal, in the sense that no productions can be excluded without obtaining a form which is not complete.

To complete the subcase analysis we first need the following notions.

Given an arbitrary alphabet $V$ define the homomorphisms $h_{i}, i=1,2$ by $h_{i}(X)=x_{i}, x$ in $V, i=1,2$. If $p$ is a set of productions we define $h_{j}(P)$ in the obvious way; we say $h_{j}(P)$ is the i-marked copy of $p$.

We now have:

Lemma 3.18

An EOL form $F=(\{S, a\},\{a\}, P, S)$ with $\{S \rightarrow a ; a \rightarrow a\} \subseteq P$ is. EOL-complete if conditions (cl') - (c3') hold.

(cI') $S \Rightarrow^{*} a^{i}, 1 \leq i \leq 3 ; S \Rightarrow^{*}$ aSS; $S \Rightarrow^{*}$ SaS; $S \Rightarrow^{*} S S a$;

$S \Rightarrow^{*}$ aSSS; $S \Rightarrow^{*}$ SaSS; $S \Rightarrow^{*}$ SSaS; S $\Rightarrow^{*}$ SSSa

via nonterminal derivations in $F$.

$\left(c 2^{\prime}\right)$ There is a natural number $k$ and there are $k-s t e p$ derivations using only $i$-marked copies of productions in $P$ : for $i=1,2$ : $s_{i} \Rightarrow a_{i} ; s_{i} \Rightarrow a_{i} s_{i} ; s_{i} \Rightarrow s_{i} a_{i} ; a_{i} \Rightarrow s_{i} ; a_{i} \Rightarrow s_{i} s_{i}$ such that for all $\alpha, \beta$ in $\left\{S_{1}, S_{2}, a_{1}, a_{2}\right\}$ * where $\alpha$ contains at least $s_{1}, s_{2}, a_{1}$ or $a_{1}, a_{2}, s_{1}$ the following holds: 
if $\alpha \Rightarrow \beta$ using these $k$-step derivations, then each intermediate word, apart possibly from $\beta$, contains at least one nonterminal $S_{1}$ or $S_{2}$.

$\left(c 3^{\prime}\right)$ The same as (c3), $F$ allows "blocking" of terminals.

Proof: Again consider an auxiliary EOL form, namely $G_{2}$ defined by the productions

$$
\begin{aligned}
& z \rightarrow a^{i} ; 1 \leq i \leq 3 ; z+a_{1} s_{1} S_{2} ; z \rightarrow s_{1} a_{1} S_{2} ; z \rightarrow S_{1} S_{2} a_{1} ; \\
& z \rightarrow a_{1} s_{1} s_{2} s_{1} ; z \rightarrow s_{1} a_{1} s_{2} s_{1} ; z \rightarrow s_{1} s_{2} a_{1} s_{1} ; z \rightarrow s_{1} s_{2} s_{1} a_{1} \\
& s_{i} \rightarrow a_{i} ; s_{i} \rightarrow s_{i} a_{j} ; s_{i} \rightarrow a_{i} s_{j} ; \\
& \left.a_{i} \rightarrow S_{i} ; a_{i} \rightarrow S_{i} S_{i} ; S_{i} \rightarrow b ; a_{i} \rightarrow b \quad\right\} \quad i=1,2 \\
& \mathrm{~b} \rightarrow \mathrm{N} ; \mathrm{N} \rightarrow \mathrm{Nb} \text {, }
\end{aligned}
$$

where $Z$ is the sentence symbol.

We claim that $\mathcal{L}_{\mathrm{s}}\left(\mathrm{G}_{1}, \Rightarrow \subseteq \mathcal{L}_{\mathrm{S}}\left(\mathrm{G}_{2}, \Rightarrow\right)\right.$, where $\mathrm{G}_{1}$ is the EOL form of Lemma 3.16. Since $G_{1}$ is $E O L-c o m p l e t e$ this implies that $G_{2}$ is EOL-complete. consider an arbitrary $G_{1}^{1} \vec{S} G_{1}$. We sketch the construction of a $G_{2}^{\prime} \underset{s}{\Delta} G_{2}$ such that $L\left(G_{2}^{\prime} \Rightarrow\right)=L\left(G_{j}^{\prime}, \Rightarrow\right)$.

For each word $x$ in $L(G j, \Rightarrow)$ with $|x| \leq 3$, it is clear that a unique isolating interpretation of the appropriate nonterminalderivations $S \Rightarrow^{*} a^{i}$, which are available by (cl') can be constructed.

For each word $x$ in $L\left(G_{j}^{\prime} \Rightarrow\right)$ with $|x|>3$ there is a $G_{j}^{\prime}$-derivation for $x$ :

$\left(^{*}\right) z^{\prime}=\alpha_{0}^{\prime} \Rightarrow \alpha_{j}^{\prime} \Rightarrow \ldots \Rightarrow \alpha_{m}^{\prime}=x, m \geq 3$

and this is the image of a $G_{1}$-derivation

$z=\alpha_{0} \Rightarrow \alpha_{1} \Rightarrow \alpha_{2} \Rightarrow \ldots \Rightarrow \alpha_{m}$

where $\alpha_{1}$ is either aS or Sa and $\alpha_{2}$ is in faSS,SaS, SSa, assS, SasS, SSaS, $S S S a\}$. Moreover by examination of $G_{1}$ the productions used in the derivation $\alpha_{2} \Rightarrow^{m-3} \alpha_{m-1}$ are taken from $S \rightarrow a ; S \rightarrow S a ; s \rightarrow$ as; $a \rightarrow S$; $a \rightarrow S S\}$ and the productions used in the one-step derivation $\alpha_{m-1} \Rightarrow \alpha_{m}$ are taken from $\{a \rightarrow b ; S \rightarrow b\}$.

We now take sufficient productions into $G_{2}^{\prime}$ so that we can "simulate" the three steps outlined above, namely (i) $Z^{\prime} \Rightarrow^{2} \alpha_{2}^{\prime}$; (ii) $\alpha_{i}^{\prime} \Rightarrow \alpha_{i+1}^{\prime}, 2 \leq i \leq m-2$, and ( $i(i) \alpha_{m-1}^{\prime} \Rightarrow \alpha_{m}^{\prime}$ (i) is obtained as a production in $G_{2}^{\prime}$, where the symbols in $\alpha_{2}^{1}$ are appropriately subscripted. (ii) again uses appropriately subscripted interpretations as does $(i i j)$. Hence corresponding to (*) we obtain a $G_{2}^{\prime}$-derivation $Z^{\prime}=\alpha_{0}^{\prime} \Rightarrow \alpha_{2}^{\prime \prime} \Rightarrow \alpha_{3}^{\prime \prime} \Rightarrow \ldots \Rightarrow \alpha_{m-1}^{\prime \prime} \Rightarrow x$ where $\alpha_{i}^{\prime \prime}$ indicates $\alpha_{i}^{\prime}$ with the appropriate subscripts. We leave the details of the construction to the reader.

Clearly $L\left(G_{2}^{\prime},\right)=L\left(G_{1}^{\prime},\right)$ and $G_{2}^{\prime} \underset{s}{\Delta} G_{2}$. Hence $G_{2}$ is EOL-complete and as $F$ expansion simulates $G_{2}, F$ is EOL-complete as desired. 


\section{Theorem 3.19}

The EPOL form

$S \rightarrow a ; S \rightarrow S S ; a \rightarrow a ; a \rightarrow S$

is EOL-complete and minimal.

Proof: We demonstrate that conditions (c $\left.1^{\prime}\right)-\left(\mathrm{c} 3^{\prime}\right)$ of Lemma 3.18 hold.

(c $\left.3^{\prime}\right)$ trivially holds.

(cl') holds since

$$
\begin{aligned}
& S \Rightarrow a ; S \Rightarrow S S \Rightarrow a a ; S \Rightarrow S S \Rightarrow a S S \Rightarrow a a ; \\
& S \Rightarrow S S \Rightarrow a S S ; S \Rightarrow S S \Rightarrow S S a \Rightarrow a a d \Rightarrow a S a \Rightarrow S a S ; \\
& S \Rightarrow S S \Rightarrow S S a ; S \Rightarrow S S \Rightarrow S S a \Rightarrow a a S \Rightarrow a S S S ; \\
& S \Rightarrow S S \Rightarrow S S a \Rightarrow a a \Rightarrow S a S S ; \\
& S \Rightarrow S S \Rightarrow a S S \Rightarrow S a a \Rightarrow S S a S ; \\
& S \Rightarrow S S \Rightarrow a S S \Rightarrow S a a \Rightarrow S S S a
\end{aligned}
$$

(c2') holds for $k=9$,

since

$$
\begin{aligned}
& s_{i} \Rightarrow a_{i} \Rightarrow s_{i} \Rightarrow a_{i} \Rightarrow s_{i} \Rightarrow a_{i} \Rightarrow s_{i} \Rightarrow a_{i} \Rightarrow s_{i} \Rightarrow a_{i}, i=1,2 ; \\
& s_{1} \Rightarrow a_{1} \Rightarrow s_{1} \Rightarrow a_{1} \Rightarrow a_{1} \Rightarrow s_{1} \Rightarrow s_{1} s_{1} \Rightarrow a_{1} a_{1} \Rightarrow s_{1} a_{1} \Rightarrow a_{1} s_{1} ; \\
& s_{2} \Rightarrow s_{2} s_{2} \Rightarrow a_{2} a_{2} \Rightarrow a_{2} s_{2} \Rightarrow s_{2} a_{2} \Rightarrow a_{2} s_{2} \Rightarrow s_{2} a_{2} \Rightarrow a_{2} s_{2} \Rightarrow s_{2} a_{2} \Rightarrow a_{2} s_{2} ; \\
& s_{1} \Rightarrow a_{1} \Rightarrow s_{1} \Rightarrow a_{1} \Rightarrow a_{1} \Rightarrow s_{1} \Rightarrow s_{1} s_{1} \Rightarrow a_{1} a_{1} \Rightarrow a_{1} s_{1} \Rightarrow s_{1} a_{1} ; \\
& s_{2} \Rightarrow s_{2} s_{2} \Rightarrow a_{2} a_{2} \Rightarrow s_{2} a_{2} \Rightarrow a_{2} s_{2} \Rightarrow s_{2} a_{2} \Rightarrow a_{2} s_{2} \Rightarrow s_{2} a_{2} \Rightarrow a_{2} s_{2} \Rightarrow s_{2} a_{2} ; \\
& a_{i} \Rightarrow s_{i} \Rightarrow a_{i} \Rightarrow s_{i} \Rightarrow a_{i} \Rightarrow s_{i} \Rightarrow a_{i} \Rightarrow s_{i} \Rightarrow a_{i} \Rightarrow s_{i}, i=1,2 ; \\
& a_{1} \Rightarrow s_{1} \Rightarrow a_{1} \Rightarrow s_{1} \Rightarrow a_{1} \Rightarrow s_{1} \Rightarrow s_{1} s_{1} \Rightarrow a_{1} a_{1} \Rightarrow a_{1} a_{1} \Rightarrow s_{1} s_{1} ; \\
& a_{2} \Rightarrow s_{2} \Rightarrow s_{2} s_{2} \Rightarrow a_{2} a_{2} \Rightarrow s_{2} a_{2} \Rightarrow a_{2} s_{2} \Rightarrow s_{2} a_{2} \Rightarrow a_{2} s_{2} \Rightarrow a_{2} a_{2} \Rightarrow s_{2} s_{2} ;
\end{aligned}
$$

and it is straightforward but tedious to check that the rest of (c2') holds. Hence the given EPOL form is EOL-complete.

To summarize this subcase we have:

\section{Theorem 3.20}

An EPOL form $F=(\{S, a\},\{a\}, P, S)$ with $\{S \rightarrow a ; a \rightarrow a ; a \rightarrow S\} \subseteq P$ and $S \rightarrow S$ not in $P$ is EOL-complete iff

$P \cap\{S \rightarrow S a ; S \rightarrow$ aS; $S \rightarrow S S\} \neq \emptyset$.

Proof: if: Fallows from Theorems 3.17 and 3.19 .

only if: If $P \cap\{S \rightarrow S a ; S \rightarrow$ a $S ; S \rightarrow S S\}=\emptyset$ then there is no nonterminal derivation for $a^{3}$ in $F$. Hence $\left\{a^{3}\right\}$ is not in $\mathcal{Z}_{s}(F, \Rightarrow)$ and therefore $F$ is not EOL-complete. 
Case $4.2 \quad\{a \rightarrow a ; S \rightarrow S\} \cap P=\emptyset$.

This subcase has not been completely resolved. We merely give two examples to illustrate both completeness and incompleteriess.

$$
\mathrm{F}_{1}: \mathrm{S} \rightarrow \mathrm{a} ; \mathrm{S} \rightarrow \mathrm{Sa} ; \mathrm{S} \rightarrow \mathrm{aS} ; \mathrm{a} \rightarrow \mathrm{S} ; \mathrm{a} \rightarrow \mathrm{SS} ; \mathrm{a} \rightarrow \mathrm{a} a
$$

is not EOL-complete, since there is no nonterminal-derivation for aa.

$$
F_{2}: S \rightarrow a ; S \rightarrow S a ; S \rightarrow a S ; S \rightarrow a a ; a \rightarrow S ; a \rightarrow S S ; a \rightarrow a a
$$

is on the other hand EOL-complete. The proof of this result makes use of the basic observation that an arbitrary EOL language $L$ can be partitioned into two EOL languages $L_{\text {even }}$ and $L_{\text {odd }}$ of even and odd

length words of $L$, respectively. Second1y Proposition 3.12 is invoked as in Lemma 3.13 above using the following observation. A word:

$$
a_{1} a_{2} \ldots a_{2 m}\left(a_{1} \ldots a_{2 m+1}\right)
$$

can be represented by, that is, compressed into, the word:

$$
\left[a_{1} a_{2}\right] \ldots\left[a_{2 m-1} a_{2 m}\right]\left(\left[a_{1} a_{2} a_{3}\right]\left[a_{4} a_{5}\right] \ldots\left[a_{2 m} a_{2 m+1}\right]\right) \text {. }
$$

Let $L_{\text {even }}$ and $L_{\text {odd }}^{\prime}$ be the "compressed" versions of $L_{\text {even }}$ and $L_{\text {odd }}$ respectively.

Now both $L_{\text {éven }}$ and $L_{\text {odd }}^{\prime}$ may be obtained as interpretations of $F_{3}: S \rightarrow a ; S \rightarrow S a ; a \rightarrow a ; a \rightarrow S$ by Theorem 3.20 above, say $F^{\prime}$ even and $F^{\prime}$ odd. Now note that for each word $x$ of length at least three in such an interpretation. the last two derivation steps use only the following two types of derivation.

$$
\mathrm{a} \Rightarrow \mathrm{S} \Rightarrow \mathrm{a}
$$

and $S \Rightarrow a \Rightarrow a$,

to produce the compressed terminals. To produce the original terminals replace these derivations by the types:

$$
\begin{aligned}
& a \Rightarrow S \Rightarrow a a, \\
& a \Rightarrow S a \Rightarrow a a, \\
& S \Rightarrow a \Rightarrow a a,
\end{aligned}
$$

and $S \Rightarrow S a \Rightarrow$ aa

as appropriate.

Aga in we leave the details to the interested reader.

In the next section we turn to short synchro-EPOL forms where the classification is more successful.

\section{III.3.2 Short $\{S, A, a\}-$ synchro-EPOL Forms}

In Theorem 3.5 the EOL-complete two-symbol synchro-EPOL forms are characterized, whereas even short two-symbol EPOL forms have not, at this time, been completely classified as has been demonstrated in 
Section 3.1. However the classification of two-symbol synchro-EPOL forms is in one sense a phoney one, since the conditions necessary for EOL-completeness (Lemma 3.4) are essentially sufficient in this case. In other words, 10oping must occur for $S$ and so must expansiveness, because the EPOL form is synchronized. Thus we turn our attention to a less restrictive case, namely short $[S, A, a]-s y n c h r o-E P O L$ forms, where the only productions for $A$ are $A \rightarrow A$ and $A \rightarrow a$. This avoids the restrictiveness of two-symbol synchro-EPOL forms by allowing $S$-productions of the form $S \rightarrow S A$ and $S \rightarrow A S$, where $A$ is now a "terminal-1ike" nonterminal. Thus the $S$-productions are a subset of the following set of productions:

$$
\begin{aligned}
P=\{S & \rightarrow A ; S \rightarrow A A ; S \rightarrow A S ; S \rightarrow S A ; \\
S & \rightarrow S ; S \rightarrow S S ; S \rightarrow a ; S \rightarrow \text { aa }\},
\end{aligned}
$$

since the $\{S, A, a\}-$ form is synchronized.

Note that there are $\{S, A, a\}-s y n c h r o-E P O L$ forms which are regular- or linear-complete (see Section 3.4).

We first prove that the $\{S, A, a\}-s y n c h r o-E P O L$ form $F=(\{S, A, a\},\{a\}, P-\{S \rightarrow S\}, S)$ is not EOL-complete. Thus, the production $S \rightarrow S$ is necessary for completeness. This leads to a general conjecture that for an arbitrary synchro-EOL form $G$ to be EOL-complete it must contain a nonterminal $B$ which is both expansive and looping. Such a result would imply that in $F$, $S$ must be both looping and expansive and hence $F$ is not EOL-complete. Unfortunately the validity of this conjecture is not yet known and so we prove the result directly for the given form $F$.

\section{Lemma 3.21}

The EOL language

$$
L=\left\{a^{2^{m}} b^{3^{n}}: m, n \geq 1\right\}
$$

does not belong to $\mathcal{L}_{s}(F, \Rightarrow)$.

Proof: First note that every context-free language belongs to $F$, because of the "terminal-like" nonterminal $A$, that is $\mathcal{L}(C F) \subseteq \mathcal{L}_{s}(F, \Rightarrow$. Also it is easy to see that languages such as

$L_{1}=\left\{a^{2^{m}} b^{n}: m, n \geq 1\right\}$ and $L_{2}=\left\{a^{3^{m}}: m \geq 1\right\}$ belong to $\mathcal{L}_{s}(F, \Rightarrow)$. We wi11 give an interpretation $F_{1}$ of $F$ such that $L\left(F_{1}, \Rightarrow\right)=L_{1}$. The case of $L_{2}$ is left to the reader.

Let $F_{1}=\left(\left\{S_{a}, S_{b}, A_{b}, a, b\right\},\{a, b\}, P, S_{b}\right)$ where $P$ is defined by: 


$$
\begin{aligned}
& S_{b} \rightarrow S_{b} A_{b} ; S_{b} \rightarrow S_{a} A_{b} ; \\
& A_{b} \rightarrow A_{b} ; A_{b} \rightarrow b ; \\
& S_{a}+S_{a} S_{a} ; S_{a} \rightarrow a a .
\end{aligned}
$$

Essentially, $b^{n}$, or rather $A_{b}^{n}$ is first generated before $a^{2^{m}}$. The reader should satisfy himself that $L\left(F_{1} \Rightarrow\right)=L_{1}$.

Turning to the proof of the Lemma, we argue by contradiction. That is, assume there is an $F^{\prime} \varangle_{s} F$ such that $L\left(F^{\prime}, \Rightarrow\right)=L$. For convenience let $L_{m}$ be defined as the subset of $L,\left\{a^{2^{m}} b^{n}: n \geq 1\right\}$, for $m \geq 1$. The spectrum of a nonterminal $B$ in $F^{\prime}$ consists of all numbers $k$ such that it is possible to derive a terminal word from $B$ in $k$ steps. The spectrum is well known to be an ultimately periodic set and, hence, we may speak of its threshold and period. The uniform period of $F^{\prime}$ is the smallest number $t$ such that for every nonterminal of $F^{\prime}, t$ exceeds the threshold of its spectrum and is divisible by its period. Now let $\alpha$ be a word derivable according to $F^{\prime}$ in less than $2 t$ steps, and let $k$ be an integer such that $0 \leq k \leq t-1$. provided $t+k$ belongs to the spectrum of each symbol in $\alpha$, we define an $0 L$ grammar $G(\alpha, k)$ as follows:

The starting word is $\alpha$. The production $B \rightarrow B$ belongs to the production set of $G(\alpha, k)$ iff $\beta$ is derivable in $t$ steps from $B$ according to $F^{\prime}$.

For each symbol $B$ in the alphabet of $G(\alpha, k)$, let $\sigma(B)$ be the set of terminal words derivable from $B$ according to $F^{\prime}$ in $t+k$ steps. Consider $\sigma$ to be a finite substitution defined on the alphabet of $G(\alpha, k) . L=L\left(F^{\prime}, \Rightarrow\right)$ can now be expressed as: (*) $L=\bigcup_{(\alpha, k)} \sigma(L(G(\alpha, k) \Rightarrow)) \cup L_{\text {finite }}$. where $L_{\text {finite }}$ is a finite language. We simply write $L(\alpha, k)$ for $\sigma(L(G(\alpha, k), \Rightarrow))$ in the sequel.

Consider a triple $(\alpha, k, m)$, where $m$ is a natural number and $\alpha$ and $k$ are as above. Given such a triple, we say $\underline{L(\alpha, k) \text { misses a final }}$ segment of $L_{m}$ if there is a number $n_{0}$ such that, whenever $n \geq n_{0}$, then the word

$$
a^{2^{m}} b^{3^{n}}
$$

is not in $L(\alpha, k)$.

We now establish the following claim. 
Claim: For each pair $(\alpha, k)$, there are only finitely many values of $m$ such that $L(\alpha, k)$ does not miss a final segment of $L_{m}$.

Since the (*)-representation above involves a finite number of pairs $(\alpha, k)$ establishing the claim implies that $L \neq L\left(F^{\prime}, \Rightarrow\right)$ for any $F^{i} \overbrace{S} F$.

In other words $L$ is not in $\mathscr{L}_{S}(F, \Rightarrow)$, by contradiction, proving the Lemma.

Proof of claim: Consider a fixed pair $(\alpha, k)$. Without loss of generality we may assume $L(\alpha, k)$ contains, for each $j \geq 1$, a word $x$ such that both $|x|_{a}>j$ and $|x|_{b}>j$. If this is not true $L(\alpha, k)$ trivialiy satisfies the claim.

Consider a fixed value of $m$ and a symbol $B$ of the system $G(\alpha, k)$. We say $B$ is $L_{m}$-good if there are infinitely many values of $n$ such that

$$
a^{2^{m}} b^{3^{n}} \text { is in } \sigma\left(B \alpha_{n}\right) \text { and } \alpha \Rightarrow^{*} \alpha_{n} \text { in } G(\alpha, k) \text {, }
$$

where $\alpha_{n}$ is a word depending on $n$.

This means that $B$ occurs as the leftmost symbol in the o-generation of arbitrarily long words of $L_{m}$. To prove the claim it is sufficient to show that no symbol is $L_{m}$-good for infinitely many values of $m$.

Assume the contrary, that is $B$ is a symbol, which is $L_{m}$-good for infinitely many values of $n$. This implies for all values of $r$ such that

$B \Rightarrow{ }^{r} B_{1}$ and $B \Rightarrow{ }^{r} B_{2}$

in $G(\alpha, k)$, that

(**) $\sigma\left(\beta_{1}\right)=\alpha\left(\beta_{2}\right)$

and $\sigma\left(\beta_{j}\right)$ consists of a single word.

If $\# \sigma\left(\beta_{\eta}\right) \neq 1$ then there is a derivation

$$
\alpha^{*} B \gamma \Rightarrow B_{1} \delta
$$

such that $a^{2^{m}} b^{3^{n}}$ is in o( $\left.\beta_{1}\right)$ for sufficiently large $m$, where $a^{2^{m}} b^{3^{n}}=$ $x_{1} x_{2}, x_{1}$ is in $\sigma\left(\beta_{1}\right)$ and $x_{2}$ is in $\sigma(\delta)$. Now there is $x_{1}^{1} \neq x_{1}$ in $\sigma\left(\beta_{1}\right)$ by assumption, and since $m$ has been chosen sufficiently large, $x_{1}^{1} x_{2}$ is not in L. (In fact choose m such that $2^{m}>\max (\{|x|: x$ in $\left.\left.\sigma\left(\beta_{1}\right)\right\}\right)$ ). By a similar argument $\sigma\left(\beta_{1}\right)=\sigma\left(\beta_{2}\right)$.

Intuitively, the terminal contribution of $B$ is determined completely by the length of the derivation.

We may now assume that $B$ is a recurring symbol, that is, in some number of steps it generates a word containing itself. If it is 
not recurring it is generated by another symbol $c$, say, which is recurring and also $\mathrm{L}_{\mathrm{m}}$-good. In this case we switch our attention to $C$. If $B$ generates a (nonterminal) word of length greater than one (according to $G(\alpha, k)$ ) in some number of steps, then the uniqueness condition (**) and the fact that $B$ is recurring imply that $B$ is not $\mathrm{L}_{\mathrm{m}}$-good for any value of $\mathrm{m}$, which is a contradiction.

Therefore $B$ must be looping, and hence $B$ is an interpretation of $A$ in the form $F$.

Consider the possible right neighbours of $B$, and their right neighbours, and so on. By a similar argument we can show that they are also interpretations of $A$. We may conclude the existence of a nonterminal $C$ such that:

(i) $C$ is an interpretation of $A$, (ii) $\sigma(c)=\{a\}$, and

(iii) for each $j \geq 1$, there is a word in $L(\alpha, k)$ containing more than $j$ occurrences of $C$ and all symbols between these occurrences are interpretations of $A$.

Conditions (i) and (iii) imply the existence of an interpretation $S^{\prime}$ of $S$ such that, for some words $\alpha_{1}, \alpha_{2}, \alpha_{3}$, possibly empty. (1) $S^{\prime} \Rightarrow \alpha_{1} \mathrm{Co}_{2} S^{\prime} \alpha_{3}$ according to $G(\alpha, k)$, where $\alpha_{1} \alpha_{2}$ contains interpretations of $A$ only. Consider words $a^{2^{m}} b^{3^{n}}$, where $m$ is sufficiently large, (1) leads to a contradiction. In other words $B$ is not $L_{m}$-good.

Hence the Claim is proved as is the Lemma.

We now have:

\section{Theorem 3.22}

A short $\{S, A, a\}-s y n c h r o-E P O L$ form $F=(\{S, A, a\},\{a\}, P, S)$ is EOL-complete iff $\{S \rightarrow S ; S \rightarrow S S\} \subseteq P$ and $P \cap\{S \rightarrow A ; S \rightarrow a\} \neq 0$.

Proof: if: Straightforward.

only if: Assume $F$ is EOL-complete. By Lemma 3.4 one of $S \rightarrow A$ or $S \rightarrow$ a must be present. Similarly $F$ must be expansive, that is, $S \rightarrow S S$ is in $F$. By Lemma 3.21 if $S \rightarrow S$ is not in $F$ then $F$ is not EOL-complete.

Thus we have completed the task we set ourselves, namely EOLcomplete short $\{S, A, a\}-s y n c h r o-E P O L$ forms have been completely characterized. 


\section{III.3.3 ETOL Forms and Completeness}

Every ETOL language can be generated by a binary propagating synchronized 2-table ETOL grammar in which one table has productions of the types:

$$
A \rightarrow B ; A \rightarrow N ; a \rightarrow B ; N \rightarrow N
$$

where $N$ is the blocking symbol, and the other table has productions of the types:

$$
A \rightarrow B ; A \rightarrow N ; A \rightarrow B C ; A \rightarrow a ; a \rightarrow N ; N \rightarrow N
$$

Hence immediately the ETOL form $G_{1}=\left(\{S, a\},\{a\}, P_{1}, P_{2}, S\right)$ where

$$
\begin{aligned}
& P_{1}=\{S \rightarrow S ; a \rightarrow S\} \text { and } \\
& P_{2}=\{S \rightarrow a ; S \rightarrow S ; S \rightarrow S S ; a \rightarrow S\}
\end{aligned}
$$

is ETOL-complete.

Analogously, the synchro-ETOL form $G_{2}$ defined by the two tables:

$$
\{S \rightarrow S ; S \rightarrow N\}
$$

and

$$
\{\mathrm{S} \rightarrow \mathrm{a} ; \mathrm{S} \rightarrow \mathrm{S} ; \mathrm{S} \rightarrow \mathrm{N} ; \mathrm{S} \rightarrow \mathrm{SS}\}
$$

is also ETOL-complete.

Note that both $G_{1}$ and $G_{2}$ have one table which if taken alone as an EOL form is in fact $E O L$-comp tete. Hence $G=\left(\{S, a\},\{a\}, P_{2}, S\right)$, an EOL-restriction of $G_{1}$ is EOL-complete.

We now demonstrate that this does not necessarily have to hold, that is, there is an ETOL form $F$ which is ETOL-complete and none of its EOL-restrictions is EOL-complete.

\section{Theorem 3.23}

$$
\begin{aligned}
& F=\left(\{S, a\},\{a\}, P_{1}, P_{2}, P_{3}, P_{4}, S\right) \text {, where } \\
& P_{1}=\{S \rightarrow a ; S \rightarrow S S ; a \rightarrow S\} \text { and } \\
& P_{2}=P_{3}=P_{4}=\{S \rightarrow a ; S \rightarrow S ; a \rightarrow a ; a \rightarrow S\}
\end{aligned}
$$

is ETOL-complete.

Exoof: Consider an arbitrary $L \subseteq \Sigma^{*}$ with $L$ in $\mathcal{X}($ ETOL). Then there exists an ETOL grammar $H=\left(V, \Sigma, Q_{1}, Q_{2}, Z\right)$ such that $L(H, \Rightarrow)=L$ and $H$ is synchronized, binary and propagating. In other words by the remarks preceding the theorem $Q_{1}$ contains productions of the types:

$$
A \rightarrow a ; A \rightarrow B ; A \rightarrow B C ; A \rightarrow N ; a \rightarrow N ; N \rightarrow N
$$

and $Q_{2}$ contains productions of the types:

$A \rightarrow B ; A \rightarrow N ; a \rightarrow B ; N \rightarrow N$

where $N$ is the blocking symbol.

We show in the following that $c L d$ is in $\mathcal{Z}_{s}(F, \Rightarrow)$, for any $c$

and $d$. Clearly this is sufficient since any $L$ in $\mathcal{Z}$ (ETOL) can be 
partitioned into

$L=\bigcup_{a, b \text { in } \Sigma} a L_{a b} b u L_{\text {finite }}$.

where $L_{a b}=\{x: a x b$ is in $L$ and $x \neq \lambda\}$ and $L_{\text {finite }}$ is the set of

remaining words of length one or two. Now $L_{a b}$ and $a L_{a b}$ b are in $\mathcal{L}$ (ETOL), since $\mathcal{L}$ (ETOL) is closed under finitetransductions and $L_{\text {finite }}$

is trivially in $\mathcal{X}(E T O L)$. Clearly $L_{\text {finite }}$ is in $\mathscr{L}_{s}\left(F, \Rightarrow\right.$ and $\mathcal{X}_{s}(F, \Rightarrow)$ is closed under finite union since it is a unary ETOL form (cf. Section II.4.2). Thus it is sufficient to show that $c L d$ is in $\mathcal{L}_{s}(F \Rightarrow \Rightarrow$ for any $c, d$.

Number the productions in $Q_{1}$ and $Q_{2}$ uniquely from 1 to $\ell=\# Q_{1}+\# Q_{2}$. We now construct an $F^{\prime} \bigotimes_{S} F$ such that $L\left(F^{\prime}, \Rightarrow\right)=c L d$. Let $F^{\prime}=\left(V^{\prime}, \Sigma^{\prime}, P_{j}^{\prime}, P_{2}^{\prime}, P_{3}^{\prime}, P_{4}^{\prime}, S^{\prime}\right)$ where

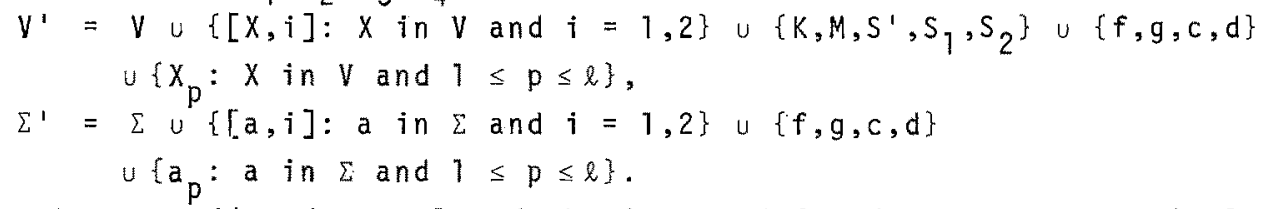
Note that $c$ and/or $d$ may already be in $\Sigma$ and $f$ and g are new terminals. Define $P_{1}$ and $P_{2}$ as follows:

(i) if $p: A \rightarrow B C$ is in $Q_{1}$, then take $[A, 1] \rightarrow A_{p}$ into $P_{2}^{\prime}$ and $A_{p} \rightarrow[B, 1][C, 1]$ into $P_{j}^{\prime}$,

(ii) if $P: A \rightarrow B$ is in $Q_{1}$, then take $[A, 1] \rightarrow a_{p}$ into $P_{2}^{\prime}$ and $a_{p} \rightarrow[B, 1]$ into $P_{1}$,

(iji) if $P: A \rightarrow$ a is in $Q_{1}$, then take $[A, 1] \rightarrow A_{p}$ into $P_{2}$ and $A_{p} \rightarrow[a, 1]$ into $P_{j}$.

(iv) if $p: a \rightarrow A$ is in $Q_{1}$, then take $[a, 1] \rightarrow a_{p}$ into $P_{2}^{\prime}$ and $a_{p} \rightarrow[A, 1]$ into $P_{i}$,

(v) if $p: A \rightarrow B$ is in $Q_{2}$, then take $[A, 2] \rightarrow a_{p}$ into $P_{2}^{\prime}$ and $a_{p} \rightarrow[B, 1]$ into $P_{1}^{\prime}$,

(vi) if $p: a \rightarrow A$ is in $Q_{2}$, then take $[a, 2] \rightarrow a_{p}$ into $P_{2}^{\prime}$ and $a_{p} \rightarrow[A, 1]$ into $P_{2}^{1}$,

(vii) take $f \rightarrow K ; M \rightarrow g$ into $P_{2}^{\prime}, K \rightarrow f ; g \rightarrow M$ into $P_{1}^{\prime}$, (viii) take $S^{\prime} \rightarrow S_{1} S_{2} ; S_{1} \rightarrow f ; S_{2} \rightarrow[Z, 1] M$ into $P_{1}^{\prime}$,

(ix) all other symbols have blocking productions $A \rightarrow N ; N \rightarrow N N$ in $P_{j}^{\prime}$ and $A \rightarrow N ; N \rightarrow N$ in $P_{2}^{\prime}$.

Let $P_{3}^{\prime}$ contain $[X, 1] \rightarrow[X, 2]$, for a.1 $X$ in $V ; f \rightarrow f ; M \rightarrow M$, and

$P_{4}^{\prime}$ contain $[X, 1] \rightarrow X$, for a $11 X$ in $V ; f \rightarrow c ; M \rightarrow d$, and in both cases all other symbols have blocking productions.

clearly $F^{\prime} \& F$. That $L\left(F^{\prime}, \Rightarrow\right)=c L d=c L(H, \Rightarrow) d$ can be seen by noting that at any intermediate step of a derivation in $F^{\prime}$, the leftmost and rightmost symbols are never both terminal unless $\mathrm{P}_{4}^{\prime}$ is applied. 
This ensures that no spurious terminal words are generated.

Secondly, an application of $P_{2}^{\prime}$ followed by $P_{j}$ is used to simulate either $Q_{1}$ or $Q_{2}$ depending on whether the initial symbols are $[x, 1]$ or $[x, 2]$ symbols. At the intermediate step the subscripts denote the unique productions to be applied by $P_{j}$. Finally $P_{3}$ enables a switch from $[X, 1]$ to $[X, 2]$ symbols to take place.

Thus $L\left(F^{\prime} \Rightarrow\right)=c L(H, \Rightarrow) d$, giving the result.

Lemma 3.24

$F=\left(\{S, a\},\{a\}, P_{1}, P_{2}, P_{3}, P_{4}, S\right)$ of Theorem 3.23 has no $E O L-$ complete EOL-restriction.

Proof: Let $F_{i}=\left(\{S, a\},\{a\}, P_{i}, S\right), i=1,2,3,4$. clearly $\mathcal{L}_{S}\left(F_{i}, \Rightarrow\right)$ $\subseteq \mathcal{L}$ (FIN) for $i=2,3,4$. Therefore consider $F_{1}$. Now aa is not in $L\left(F_{1} \Rightarrow\right)$, hence $F_{1}$ is not $E O L$-complete.

We will show as the final theorem of this section that there is a two-tabled ETOL form, which is ETOL-complete and has no EOL-complete EOL-restriction. Along the way we will also demonstrate that there are three-tabled ETOL forms with this property.

observe that $G_{3}=\left(\{S, a\},\{a\}, P_{1}, P_{2}, S\right)$ where

and

$$
P_{1}=\{S \rightarrow a ; S \rightarrow S ; S+S S ; a \rightarrow S\}
$$

$$
P_{2}=\{S \rightarrow S ; a \rightarrow a\}
$$

is ETOL-complete. This follows from the construction of a two-tabled ETOL gramar equivalent to a given synchronized, binary and propagating ETOL grammar. clearly the construction preserves the properties of being binary and separated and propagating. The property of synchronization is also preserved if table-switching productions are only included for the original nonterminals. This is no loss of generality since terminals only have blocking productions in the original grammar. Recalling that the two tables in the new grammar only block terminals, while only the non-switching table introduces them, allowing the switching table to have the productions $a \rightarrow$ a for all a in $\Sigma$, does not affect the generated 1 anguage. Therefore $G_{3}$ above is ETOL-complete.

We first take a diversion and consider an EOL-complete ETOL form none of whose EOL-restrictions is EOL-complete. 
Theorem 3.25

$$
\begin{aligned}
& \quad F=\left(\{S, a\},\{a\}, P_{0}, P_{1}, S\right) \text { where } \\
& P_{0}=\{S \rightarrow S ; S \rightarrow S S ; a \rightarrow a\} \text { and } \\
& P_{1}=\{S \rightarrow a ; a \rightarrow a\}
\end{aligned}
$$

is EOL-complete. Moreover neither EOL-restriction is EOL-complete.

Proof: The last sentence is trivial since $L\left(F_{0}, \Rightarrow\right)=\emptyset$ and $L\left(F_{1}, \Rightarrow\right.$ is in $\mathcal{L}(F I N)$.

Clearty $\mathcal{L}(E O L) \subseteq \mathcal{L}_{\mathrm{s}}(\mathrm{F}, \Rightarrow$ since every EOL language $L$ can be generated by a binary, propagating and synchronized EOL grammar $G=(V, \Sigma, P, Z)$, and it is straightforward to construct an $F^{\prime}{ }_{S} F$ with $P_{0}^{\prime}=\{A \rightarrow \alpha: A \rightarrow \alpha$ in $P, \alpha$ not in $\Sigma\}$

$u\{a \rightarrow a: a$ in $\Sigma\}$

and $P_{1}^{\prime}=\{A \rightarrow a: A \rightarrow a$ in $P$, a in $\Sigma\}$

$u\left\{a \rightarrow a: a\right.$ in $\left.\Sigma_{i}\right\}$

such that $L\left(F^{\prime}, \Rightarrow\right)=L(G, \Rightarrow)$.

Conversely, we show that $\mathcal{L}_{S}(F, \Rightarrow) \subseteq \mathcal{L}(E O L)$. Consider any interpretation $F^{\prime}=\left(V^{\prime}, \Sigma^{\prime}, P_{0}^{\prime}, P_{j}^{\prime}, S^{\prime}\right) \varangle_{S} F_{\text {with }} \Sigma^{\prime}=\left\{a_{1}, \ldots, a_{t}\right\}$, for some $t \geq 1$.

For any bit sequence $x$, that is $x$ is in $\{0,1\}$ * define a substitution $f_{x}$ on $\Sigma^{\prime *}$ as follows: for all a in $\Sigma^{\prime}$, for a $11 \times$ in $\{0,1\}$ * if $x=\lambda, f_{x}(a)=\{a\}$

if $x=0$ or $1, f_{x}(a)=\left\{b: a \rightarrow b\right.$ is in $\left.p_{x}^{1}\right\}$, and

if $x=i y, i=0$ or $1, f_{i y}(a)=f_{y}\left(f_{i}(a)\right)$.

Essentially $f_{x}(a)$ is the set of all terminals obtainable from a by the table sequence $x$.

Now \#f $x(a) \leq \# \Sigma^{\prime}$ for al $1 x$ in $\{0,1\}$ * hence there are on $1 y$ finitely many different substitutions of the form $f_{x}$. Let these substitutions be $f_{x_{1}}, \ldots, f_{x_{r}}$.

Construct $F^{\prime \prime}=\left(V^{\prime}, \Sigma^{\prime}, P_{0}^{\prime \prime}, P_{j}^{\prime \prime}, S^{\prime}\right){ }_{S}^{\Delta} F$ such that $P_{i}^{\prime \prime}$ contains the same productions for nonterminals as $P_{i}^{\prime}$ and the only productions for terminals being $a \rightarrow a$.

$$
\text { Now } \begin{aligned}
L\left(F^{\prime} \Rightarrow\right) & =\bigcup_{x \text { in }\{0,1\} * x} f_{x}\left(L\left(F^{\prime \prime}, \Rightarrow\right)\right) \\
& =\bigcup_{i=1}^{r} f_{x_{i}}\left(L\left(F^{\prime \prime}, \Rightarrow\right)\right) .
\end{aligned}
$$

since $L\left(F^{\prime \prime} \Rightarrow\right.$ is in $\mathcal{L}(E O L)$ and $\mathcal{L}(E O L)$ is closed under finite union and finite substitution, $L\left(F^{\prime}, \Rightarrow\right.$ is in $\mathcal{L}(E O L)$ as desired. Hence the theorem. 
This theorem is of interest on two counts. First, the only productions for terminals in $F$ are $a \rightarrow a$. This should be compared with Lemma 3.2 which asserts that an EOL-complete EOL form must have a production $a \rightarrow \alpha$ with a containing a nonterminal. Clearly this is just not true here.

Secondly, we can now derive a new normal form for ETOL grammars on the basis of this theorem.

\section{Theorem 3.26}

$F=\left(\{S, a\},\{a\}, P_{0}, P_{1}, P_{2}, S\right)$, where $P_{0}$ and $P_{1}$ are the same as in Theorem 3.25 and $P_{2}=\{S \rightarrow S ; a \rightarrow a\}$, is ETOL-complete.

Exoof: Consider an arbitrary synchronized, propagating and binary ETOL grammar $G=\left(V, \Sigma, Q_{1}, Q_{2}, Z\right)$. We construct an $F^{\prime}=\left(V, \Sigma, P_{0}^{\prime}, P_{1}^{\prime}, P_{2}^{\prime}, S^{\prime}\right) \triangle_{S} F$ such that $L\left(F^{\prime} \Rightarrow\right)=L(G, \Rightarrow)$. This implies $F$ is ETOL-complete.

We may assume, as before, that:

$Q_{1}$ contains only productions of the types:

$$
A \rightarrow a ; A \rightarrow B ; A \rightarrow B C ; a \rightarrow N ; N \rightarrow N
$$

and $Q_{2}$ only productions of the types:

$$
\mathrm{A} \rightarrow \mathrm{B} ; \mathrm{A} \rightarrow \mathrm{N} ; \mathrm{a} \rightarrow \mathrm{N} ; \mathrm{N} \rightarrow \mathrm{N} .
$$

Proceed as in the proof of Theorem 3.25, that is

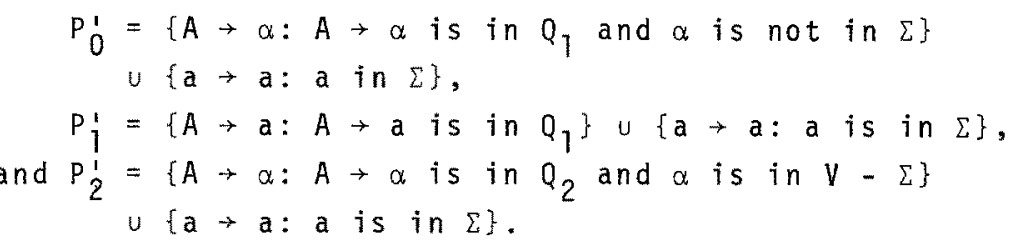

clearly $L\left(F^{\prime}, \Rightarrow\right)=L(G, \Rightarrow)$ hence $F$ is ETOL-complete.

Again note that no EOL-restriction of $F$ is EOL-complete.

We terminate this section by demonstrating that there is indeed a two-tabled ETOL form which is ETOL-complete and none of its EOL-restrictions is EOL-complete.

\section{Theorem 3.27}

and

Let $F=\left(\{S, a\},\{a\}, P_{1}, P_{2}, S\right)$, where

$P_{1}=\{S \rightarrow S ; S \rightarrow S S ; a \rightarrow S\}$

$$
P_{2}=\{S \rightarrow a ; S \rightarrow S ; a \rightarrow S\} .
$$

Then $F$ is ETOL-complete and moreover neither EOL-restriction is EOLcomplete. 
Proof: Clearly each EOL-restriction is not EOL-complete. Let $G=\left(V, \Sigma, Q_{1}, Q_{2}, Z\right)$ be the ETOL grammar in the proof of the previous theorem. Then $Q$, only contains productions of the types:

$$
A \rightarrow a ; A \rightarrow B ; A \rightarrow B C ; a \rightarrow N ; N \rightarrow N
$$

and $Q_{2}$ only the types:

$A \rightarrow B ; A \rightarrow N ; a \rightarrow N ; N \rightarrow N$.

Construct $F^{\prime}=\left(V, \Sigma, P_{1}^{\prime}, P_{2}^{\prime}, S^{\prime}\right) \Delta_{S}^{\Delta} F$, where

$$
\begin{aligned}
P_{1}^{\prime} & =\left\{A \rightarrow \alpha: A \rightarrow \alpha \text { in } Q_{1} \text { and } \alpha \text { not in } \Sigma\right\} \\
& u\{a \rightarrow N: \text { a is in } \Sigma\} \\
& u\{N \rightarrow N\},
\end{aligned}
$$

and $P_{2}^{\prime}=\left\{A \rightarrow a: A \rightarrow\right.$ a is in $Q_{1}$

$u\left\{X \rightarrow Y: X \rightarrow Y\right.$ is in $Q_{2}, X, Y$ in $\left.V-\Sigma\right\}$

$u\{a \rightarrow N: a$ is in $\Sigma\}$

clearly $L\left(F^{\prime}, \Rightarrow\right)=L(G, \Rightarrow)$, hence the result.

Having resolved the main problem of this section, it only remains to mention that no classification of two-symbol-ET0L forms has been attempted at the time of writing.

\section{III.3.4 Regular-completeness}

We will demonstrate in this section that there are regularcomplete EOL forms. In particular the form

$\mathrm{H}: \mathrm{S} \rightarrow \mathrm{a} ; \mathrm{S} \rightarrow \mathrm{Sa} ; \mathrm{a} \rightarrow \mathrm{a}$

is regular-complete.

At the time of writing there is no classification of two-symbol EOL forms with regard to regular-completeness.

\section{Pheorem 3.28}

$$
\mathrm{H}: \mathrm{S} \rightarrow \mathrm{a} ; \mathrm{S} \rightarrow \mathrm{Sa} ; \mathrm{a} \rightarrow \mathrm{a}
$$

is regular-complete.

Proof: Clearly $\mathcal{L}(R E G) \subseteq \mathcal{L}_{5}(H, \Rightarrow)$, hence it remains to establish the converse inclusion.

Consider an arbitrary $H^{\prime}=(V, \Sigma, P, S) \underset{S}{A} H(\mu)$. It is sufficient to demonstrate that $L\left(H^{\prime} \Rightarrow \Rightarrow\right.$ is in $\mathcal{L}$ (REG).

First construct the context-free grammar $G=\left(V, \Sigma, P^{\prime}, S\right)$

where $P^{\prime}=P \cap\left((V-\Sigma) \times V^{*}\right)$, clearly $L(G, \Rightarrow)$ is in $\mathcal{L}(R E G)$.

Second construct a gsm $M$ such that $M(L(G, \Rightarrow))=L\left(H^{\prime}, \Rightarrow\right)$.

This immediately implies $L\left(H^{\prime} \Rightarrow \Rightarrow\right.$ is in $\mathcal{L}(R E G)$ since $\mathcal{L}(R E G)$ is closed under gsm maps. 
Suppose $\Sigma=\left\{a_{1}, \ldots, a_{r}\right\}$.

Let $R_{n}\left(a_{i}\right)=\left\{a_{j}: a_{i} \Rightarrow^{n} a_{j}\right.$ in $\left.H^{\prime}\right\}$ for each $n \geq 0$ and all $i, 1 \leq i \leq r$, informally $R_{n}\left(a_{i}\right)$ is the set of terminals reachable from $a_{i}$ in $n$ steps. Let $T_{n}$ be the $r$-tuple $\left[R_{n}\left(a_{1}\right), \ldots, R_{n}\left(a_{r}\right)\right]$ for all $n \geq 0$. Observe that there are only a finite number of distinct $r$-tuples. Define the gsm $M=(Q, \Sigma, \Sigma, \delta, Z)$ as follows:

$Q=\left\{T_{i}: i \geq 0\right\}, \delta$ is defined by:

for all $T_{j}$ in $Q$ and $a_{j}$ in $\Sigma$,

$$
\delta\left(T_{i}, a_{j}\right)=\left\{\left(T_{i+1}, a_{k}\right): a_{k} \text { is in } R_{i}\left(a_{j}\right)\right\} \text {, }
$$

and $Z=Q$ is the set of start states. Note that this nonstandard gsm can be converted into a standard one without too much difficulty, however the definition used here is convenient.

It remains to show that $M(L(G, \Rightarrow))=L\left(H^{\prime} \Rightarrow\right)$.

(i) $L\left(H^{\prime}, \Rightarrow\right) \subseteq M(L(G, \Rightarrow))$.

Suppose $b_{1} \ldots b_{\ell}$ is in $L\left(H^{\prime}, \Rightarrow\right.$ then there is a derivation:

$$
\begin{aligned}
S & \Rightarrow A_{\ell} c_{\ell} \Rightarrow A_{\ell-1} c_{\ell-1} c_{\ell}^{(1)} \Rightarrow \ldots \\
& \Rightarrow A_{2} c_{2} c_{3}(1) \ldots c_{\ell}^{(\ell-2)} \Rightarrow c_{1} c_{2}{ }^{(1)} c_{3}(2) \ldots c_{\ell}^{(\ell-1)} \Rightarrow b_{1} \ldots b_{\ell}
\end{aligned}
$$

Thus $c_{1} \Rightarrow^{n} b_{1}=c_{2} \Rightarrow^{n+1} b_{2}, \ldots, c \Rightarrow^{n+l-1} b_{\ell}$, in other words

$$
b_{1} \text { is in } R_{n}\left(c_{1}\right), b_{2} \text { is in } R_{n+1}\left(c_{2}\right), \ldots, b_{\ell} \text { is in } R_{n+\ell-1}\left(c_{\ell}\right) \text {. }
$$

Now by construction $c_{1} \ldots c_{l}$ is in $L(G, \Rightarrow)$. With $T_{n}$ as the start state

$$
\left(c_{1} \ldots c_{\ell}, T_{n}, \lambda\right) \longmapsto\left(c_{2} \ldots c_{\ell}, T_{n+1}, R_{n}\left(c_{1}\right)\right) \longmapsto\left(c_{3} \ldots c_{\ell}, T_{n+2}, b_{1} R_{n+1}\left(c_{2}\right)\right)
$$

hence $b_{1} \ldots b_{\ell}$ is in $M(L(G, \Rightarrow))$.

$$
\cdots \mapsto\left(\lambda, T_{n+\ell}, b_{1} \ldots b_{\ell-1} R_{n+\ell-1}\left(c_{\ell}\right)\right)
$$

(ii) $M(L(G, \Rightarrow)) \subseteq L\left(H^{\prime}, \Rightarrow\right)$.

This follows similarly.

Obviously

$$
S \rightarrow a ; S \rightarrow a S ; a \rightarrow a
$$

is also regular-complete.

We conjecture that if a given unary $E O L$ form $H=(V,\{a\}, P, S)$

is (i) stable with respect to terminals, that is, a $\rightarrow$ a is the only production for a in $P$, and $(i j)$ the context-free grammar $G$ obtained from $H$ by removing $a \rightarrow$ a from $P$ has $\mathcal{L}_{S}(G, \Rightarrow)=\mathcal{L}(R E G)$, then $H$ is also regular-complete.

The condition of stability is important, since for example,

$$
S \rightarrow a ; S \rightarrow a S ; a \rightarrow a ; a \rightarrow S
$$

is not regular-complete. This can be seen by considering the following EOL grammar 
$\mathrm{F}: \mathrm{S} \rightarrow \mathrm{aB} ; \mathrm{B} \rightarrow \mathrm{CB} ; \mathrm{B} \rightarrow \mathrm{b} ; \mathrm{a} \rightarrow \mathrm{B} ; \mathrm{c} \rightarrow \mathrm{c} ; \mathrm{b} \rightarrow \mathrm{N} ; \mathrm{N} \rightarrow \mathrm{N}$.

It is straightforward to see that $L(F, \Rightarrow)=\left\{c^{i} b c^{i+1} b: i \geq 0\right\}$, which is, of course, non-reguiar. 


\section{III.4 Goodness and Very-completeness}

Taking the concept of completeness (studied in the previous section) further we arrive at the notions of very completeness, abbreviated vompleteness, and goodness. There is little general theory here, rather a number of examples are given, which are shown to exhibit specific properties.

Section 4.1 introduces the basic notions and provides some preliminary results. EOL forms are studied in section 4.2 while ETOL forms are considered in Section 4.3. Finally, in section 4.4 mutual and relative goodness are discussed, albeit briefly.

\section{III.4.1 Preliminaries}

In the previous section a study was made of forms $F$ with the property that $\mathcal{L}_{S}(F, \Rightarrow)=\mathcal{L}(E O L)$. In the case this holds $F$ is said to be EOL-complete. Looking at this definition from another vantage point we can say that $F$ is able to generate one EOL-grammatical family, namely $\mathcal{L}$ (EOL) itself. We now generalize this to

$F$ is EOL-very-complete (EOL-vomplete) ifi for every EOL-grammatical family $\mathcal{Z}$ there exists an interpretation $F^{\prime} \underset{S}{\Delta} F$ such that $\chi_{s}\left(F^{\prime}, \Rightarrow\right)=\mathcal{X}$.

Thus if $F$ is EOL-vomplete not only is it able to generate $\mathcal{Z}(E O L)$ but also via interpretation all EOL-grammatical families can be generated.

By the results of Section 2 we know that every EOL form $G$ has a form equivalent $E O L$ form $H$ in binary normal form, that is, $H$ only has productions of the types:

$$
\begin{aligned}
& A \rightarrow B ; A \rightarrow B C ; A \rightarrow a ; A \rightarrow \lambda ; \\
& a \rightarrow a ;
\end{aligned}
$$

Hence immediately we have our first result, namely EOL-vomplete forms exist.

Theorem 4.1

$$
F: S \rightarrow S ; S \rightarrow S S ; S \rightarrow a ; S \rightarrow \lambda ; a \rightarrow S
$$

is EOL-vomplete.

Proof: Each EOL form $H$ in binary normal form is an interpretation of $\mathrm{F}$.

Again shifting our vantage point, given an EOL-vomplete form $F$, vompleteness means that for every EOL-grammatical family $\mathcal{Z}$ such that 
$\mathcal{Z}^{\circ} \leq \mathcal{Z}_{\mathrm{s}}(\mathrm{F} \Rightarrow)$ there is an $\mathrm{F}^{\prime} \Delta \mathrm{F}$ with $\mathcal{Z}_{\mathrm{s}}\left(\mathrm{F}^{\prime} \Rightarrow \Rightarrow\right)=\mathscr{Z}$. The condition "such that $\mathcal{Z} \subseteq \mathcal{L}_{s}(F, \Rightarrow)$ " is vacuously satisfied here. However, this leads us to the notion of goodness.

We say an EOL form $F$ is good if for all EOL-grammatical families $\mathcal{Z} \subseteq \mathcal{L}_{s}\left(F, \Rightarrow\right.$ there is an $F^{\prime} \Delta \vec{s}$ such that $\mathscr{Z}_{s}\left(F^{\prime}, \Rightarrow\right)=\mathcal{X}^{\circ}$.

In particular an EOL form is EOL-vomplete if it is both EOLcomplete and good.

If $F$ is not good it is said to be bad. Since Theorem 4.1 demonstrates the existence of vomplete EOL forms, we exhibit and EOL form which is good but not EOL-complete.

\section{Theorem 4.2}

$F: S \rightarrow a ; a \rightarrow a$ is good and not complete.

Proof: First note that $\mathcal{L}_{S}(F, \Rightarrow)=\mathcal{L}(A L P H)$, the family of finite alphabets. Hence $F$ is not EOL-complete. It remains to prove that $F$ is good. Let $\mathcal{L}(k)$ be the subfamily of $\mathcal{L}(A L P H)$ consisting of 1 anguages with at least $k>0$ elements. Hence $\mathcal{Z}(1)=\mathcal{L}$ (ALPH). We now establish the following claim.

Claim: If $G$ is an EOL form such that $\mathcal{L}_{S}(G, \Rightarrow \subseteq \mathcal{L}$ (ALPH) then $\mathcal{L}_{\mathrm{s}}(G, \Rightarrow)=\mathcal{L}(k)$ for some $k>0$.

Proof of claim: clearly, only the cardinality of the sets in $\mathcal{L}_{s}(G, \Rightarrow)$ is essential rather than the names of the symbols, since we are dealing with languages which are alphabets.

Now assume $\left\{a_{1}, \ldots, a_{m}\right\}$ is in $\mathcal{L}_{s}(G, \Rightarrow), m>0$. Then

$\left\{a_{1}, \ldots, a_{m+1}\right\}$ is in $\mathcal{Z}_{s}(G, \Rightarrow)$ for $a l 1 a_{m+1} \neq a_{i}, 1 \leq i \leq m$. Letting $\left\{a_{1}, \ldots, a_{m}\right\}=L\left(G^{\prime}, \Rightarrow\right)$ for some $G^{\prime} \underset{s}{a} G$, we can construct $G^{\prime \prime} \underset{s}{\Delta} G^{\prime}$ such that $L\left(G^{\prime \prime}, \Rightarrow\right)=\left\{a_{1}, \ldots, a_{m+1}\right\}$ as follows.

For each production in $G$ : containing $a_{m}$ make a copy in which $a_{m}$ is replaced throughout by $a_{m+1}$. Now include all the productions of $G^{\prime}$ together with the "copy" productions in $G$ ". Clearly $L(G ", \Rightarrow)=$ $\left\{a_{1}, \ldots, a_{m+1}\right\}$ and $G^{\prime \prime} a_{5} G^{\prime}$.

Thus we have shown that if $\mathcal{Z}_{\mathrm{s}}(G, \Rightarrow)$ contains a language with $m$ symbols, $m>0$, then it contains a 11 alphabets with cardinality at least $m$. Hence $\mathcal{L}_{\mathrm{s}}(\mathrm{G} \Rightarrow)=\mathcal{X}(\mathrm{k})$ for some $k>0$. Thus the claim is established. 
To prove the theorem it is only necessary to demonstrate that there exist $F_{k} \vec{s} F$ with $\not{Z}_{s}\left(F_{k} \Rightarrow\right)=\left\{(k), k>0\right.$. clearly $F$ is $F_{j}$. In general, $F_{k}$ is defined by productions:

$$
S \rightarrow a_{1} ; a_{j} \rightarrow a_{i+1}, 1 \leq i<k ; a_{k} \rightarrow a_{k}
$$

clearly $F_{k} \underset{s}{\Delta} F$ and $Z_{s}\left(F_{k} \Rightarrow\right)=\alpha(k)$.

To close this introductory section we establish the existence of bad EOL forms.

\section{Theorem 4.3}

$$
F: S \rightarrow a ; a \rightarrow N ; N \rightarrow N \text { is bad. }
$$

Proof: The families $\mathcal{L}(k)$ of the previous theorem are all subfamilies of $\mathcal{Z}_{s}(F, \Rightarrow)$. However, since $F$ is synchronized, for each $F^{\prime} \triangleleft F$, $\mathcal{L}_{s}\left(F^{\prime} \Rightarrow\right.$ contains a non-empty language and hence by simulation it also contains a singleton language. In other words $\mathscr{L}_{\mathrm{s}}\left(F^{\prime} \Rightarrow \Rightarrow\right)=\mathcal{L}(1)$ for all $F^{\prime}$ and therefore for a $11 \mathrm{k} \geq 2, \mathcal{L}(k) \neq \mathcal{L}_{s}\left(F^{\prime} \Rightarrow\right)$. Therefore $F$ is bad. $\square$

Notice that $F$ of Theorem 4.3 is as bad as it could be, that is, the only EOL-grammatical family it generates is $\mathcal{Z}_{\mathrm{s}}(F, \Rightarrow)$ and no other.

\section{III.4.2 The EOL Situation}

In Section 1 we have given examples of badness, goodness and EOL-vompleteness. In the present section we give results, which are more generally applicable. Thus it is shown that each synchronized EOL form is bad, and that each propagating EOL form is not EOL-vomplete. Finally, we consider the languages of EOL forms and questions of inherent goodness and badness.

Turning to our first result we have:

\section{Theorem 4.4}

Let $F=(V, \Sigma, P, S)$ be a synchronized $E O L$ form and $L(F, \Rightarrow)$ contain at least one nonempty word. Then $F$ is bad and in particular no synchronized EOL form is EOL-vomplete.

Proof: We will exhibit an EOL-grammatical family $\mathcal{L}$ such that $\mathcal{L} \leq \mathcal{L}_{\mathrm{s}}\left(\mathrm{F}, \Rightarrow\right.$ but $\mathcal{Z} \neq \mathcal{L}_{\mathrm{s}}\left(\mathrm{F}^{\prime} \Rightarrow\right.$ for all $\mathrm{F}^{\prime} \underset{\mathrm{s}}{\mathrm{F}} \mathrm{F}$.

Let $x$ be in $L(F, \Rightarrow), x \neq \lambda$. Let a $] p h(x)=\Delta=\left\{a_{1}, \ldots, a_{m}\right\}, m \geq 1$. Construct an $E O L$ form $G=\left(\{Z, N\} \cup \Delta \cup \Delta^{\prime}, \Delta \cup \Delta^{\prime}, R, Z\right)$ where 
$\Delta^{\prime}=\left\{a^{\prime}: a\right.$ in $\left.\Delta\right\}$ and

$R=\{Z \rightarrow x\} \cup\left\{a_{i} \rightarrow a_{i}^{\prime}: 1 \leq i \leq m\right\} \cup\left\{a_{i}^{i} \rightarrow N: 1 \leq i \leq m\right\} \cup\{N \rightarrow N\}$.

Now for each $G^{\prime} \Delta_{S} G(\mu), L\left(G^{\prime}, \Rightarrow\right.$ contains at least two words, since we have used terminal forcing in $G$ to ensure this. Further

$L\left(G^{\prime} \Rightarrow\right) \subseteq \mu(L(G, \Rightarrow))$, by definition. Thus we can construct an interpretation $F^{\prime} \leftrightarrow_{S} F$ with $L\left(F^{\prime} \Rightarrow \Rightarrow\right) L\left(G^{\prime} \Rightarrow\right)$, since $F$ is synchronized. In other words $\mathcal{Z}_{\mathrm{s}}^{\mathrm{s}}(\mathrm{G}, \Rightarrow) \subseteq \mathcal{Z}_{\mathrm{S}}(\mathrm{F}, \Rightarrow)$.

However $\mathcal{L}_{S}\left(G, \Rightarrow \neq \mathcal{L}_{S}\left(F^{\prime}, \Rightarrow\right)\right.$ for any $F^{\prime} \Delta_{S} F$ since $F$ and therefore $F^{\prime}$ are both synchronized. Hence $F$ is bad.

We can generalize this result substantially as follows. Let $F=(V, \Sigma, P, S)$ be an EOL form. We say $F$ is k-synchronized, $k>0$, if

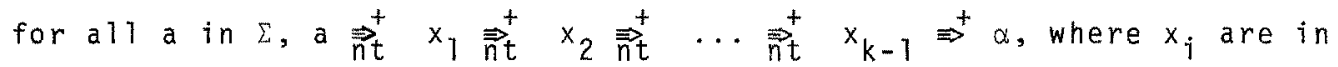
$\Sigma^{+}, 1 \leq i<k$, implies $\alpha$ is not in $\Sigma^{*}$. Hence $k$-synchronization is a generalization of synchronization, in particular "l-synchronized" is the same as "synchronized".

Now using the same proof techniques as in Theorem 4.4, namely construct an EOL form $G$ such that $S \Rightarrow x \Rightarrow x_{1} \Rightarrow \ldots \Rightarrow x_{k} \Rightarrow$ blocking in $G$. Then each language in $\mathcal{L}_{s}(G, \Rightarrow)$ contains at least $k+1$ words while for each $k$-synchronized form $F$, there is an $L$ in $\mathcal{L}_{S}(F, \Rightarrow$ containing as few as $k$ words. This gives:

Corozzary 4.5

Let $F$ be a k-synchronized EOL form, $k>0$ such that $L(F, \Rightarrow)$ contains at least one non-empty word. Then $F$ is bad.

We now turn to propagating EOL forms and prove that no propagating EOL form is EOL-vomplete.

Theorem 4.6

Let $F=(V, \Sigma, P, S)$ be a propagating $E O L$ form. Then $F$ is not EOL-vomplete.

Proof: We first establish the following claim.

Claim: No propagating EOL form is form equivalent to the EOL form $G$ defined by the productions:

$\mathrm{S} \rightarrow \mathrm{aba} ; \mathrm{a} \rightarrow \mathrm{cd} ; \mathrm{b} \rightarrow \lambda ; \mathrm{c} \rightarrow \mathrm{c} ; \mathrm{d} \rightarrow \mathrm{d}$. 
Proof: $L=L(G, \Rightarrow)=\{a b a, c d c d\}$. Note that each language in $\mathscr{L}_{s}(G, \Rightarrow)$ contains at least two words, one of length three and one of length four. Assume $H=(U, \Sigma, R, Z)$ is a propagating EOL form which is form equivalent to $G$. Then there exists an $H^{\prime}=\left(U^{\prime}, \Sigma^{\prime}, R^{\prime}, Z^{\prime}\right) \underset{S}{\Delta} H$ with $L\left(H^{\prime}, \Rightarrow\right)=L$. Consider a shortest derivation of cdcd in $H^{\prime}$, that is:

D: $z=\alpha_{0} \Rightarrow \alpha_{1} \Rightarrow \ldots \Rightarrow \alpha_{m}=$ cdcd.

Since $H^{\prime}$ is propagating cdcd $\Rightarrow$ aba is impossible. Secondly, no terminal word other than aba can occur in $D$. On the one hand if $\alpha_{i}=c d c d$ for some $i<m$, then $D$ is not a shortest derivation of cdcd. on the other hand if $\alpha_{i}$ is terminal for some $i<m, \alpha_{i} \neq a b a$ and $\alpha_{i} \neq c$ cdcd then $L\left(H^{\prime}, \Rightarrow\right) \neq L$. This leaves two cases to consider.

(i) Assume aba occurs in $D$. Then we have:

$D: Z=\alpha_{0} \Rightarrow$ aba $\Rightarrow^{k}$ cdcd.

Now consider the contributions of $a$ and $b$ to cdcd. There are three cases:

(a) $a \Rightarrow^{k} c, b \Rightarrow^{k} d, a \Rightarrow^{k} c d$;

(b) $a \Rightarrow^{k} c, b \Rightarrow^{k} d c, a \Rightarrow^{k} d$;

(c) $a \Rightarrow^{k} c d, b \Rightarrow^{k} c, a \Rightarrow^{k} d$;

In case (a) we also have

$a b a \Rightarrow c d c$.

in case (b)

$a b a \Rightarrow c d c c$

and in case (c)

$a b a \Rightarrow d c d$,

thus in all cases $L\left(H^{\prime}, \Rightarrow\right) \neq L$.

(ii) Assume aba does not occur in $D$. Then there is an $H^{\prime \prime} \triangleleft H_{S}$ ' whose only derivation is

$Z \stackrel{m}{m} \mathrm{cdcd} \Rightarrow+\ldots$

such that $L\left(H^{\prime \prime} \Rightarrow\right)=\{c d c d\}$. But this contradicts the initial observation that each language in ${ }_{S}(G, \Rightarrow)$ contains a word of length three and a word of length four.

Therefore in all cases we have arrived at a contradiction and the claim is proved.

Returning to the theorem, assume $F$ is EOL-vomplete. Then there exists $F^{\prime} \&_{s} F$ with $\mathcal{L}_{s}\left(F^{\prime}, \Rightarrow\right)=\mathcal{L}_{s}(G, \Rightarrow)$ of the claim. However since $F$ is propagating, $F^{\prime}$ is also propagating and this contradicts the claim. Hence the theorem is proved. 
The claim of Theroem 4.6 also gives a more general result, namely:

\section{Corozzary 4.7}

Let $F$ be $S \rightarrow a b a ; a \rightarrow c d ; b \rightarrow \lambda ; c \rightarrow c ; d \rightarrow d$ and $H$ be $a$ propagating EOL form with $\mathcal{L}_{S}(F, \Rightarrow) \subseteq \mathcal{L}_{S}(H, \Rightarrow)$.

Then $H$ is bad.

However the results on propagating EOL forms are clearly weaker than those for synchronized EOL forms. At the time of writing Tittie is known of the goodness of propagating EOL forms. However a recent result has demonstrated that an EOL-complete form which has terminal erasing but rio nonterminal erasing is not EOL-vomplete. This strengthens Theorem 4.6 considerably.

Finally, we consider the notions of inherent goodness and badness of EOL languages rather than forms.

Let $L$ be an EOL 1anguage. We say $L$ is (inherently) good if $L=L(F, \Rightarrow)$ for some good EOL form $F$. Similarly we say $L$ is (inherently) bad if there is no good EOL form $F$ with $L(F, \Rightarrow)=L$.

By the results above $\{a\}$ and $\{a\}^{*}$ are both good. More generally we have:

\section{Theorem 4.8}

Let $L \subseteq \Sigma^{*}$ be an EOL language and $\{a\} * \subseteq L$, for some a in $L$. Then $L$ is good.

Proof: Let $G=(V, \Sigma, P, S)$ be synchronized $E 0 L$ grammar with $L=L(G, \Rightarrow)$. Construct an $E O L$ form $F=(U, \Sigma, R, Z)$ from $G$ as follows:

$$
\begin{aligned}
& U=V \cup\{Z\} \text { and } \\
& R=P \cup\{Z \rightarrow S ; Z \rightarrow Z ; Z \rightarrow \lambda ; Z \rightarrow a ; Z \rightarrow Z Z ; a \rightarrow Z\}
\end{aligned}
$$

where $\{a\}^{*} \subseteq L$.

Clearly $L \subseteq L(F, \Rightarrow)$. Moreover $L(F, \Rightarrow) \subseteq L$ since whenever a word $a^{i}$ is generated from either $S$ or $Z$ its only terminal successors are words in $\{a\}$ * from productions in R-P. On the other hand any word in $L(F, \Rightarrow)-\{a\} *$ has no successor terminal words.

$$
\text { Finally } F^{\prime}: Z \rightarrow \lambda ; Z \rightarrow a ; Z \rightarrow Z ; Z \rightarrow Z Z ; a \rightarrow Z \underset{s}{a} F \text { and } F^{\prime} \text { is }
$$
EOL-vomplete, hence the result. 
Conversely, we have:

\section{Theorem 4.9}

$L=\{a, a a\}$ is bad.

Proof: Assume $L$ is good. Then there is a good EOL form $F$ with $L(F, \Rightarrow)=L$. Now by Theorem $4.4 \mathrm{~F}$ is not synchronized. Letting $F=(V,\{a\} \cup \Sigma, P, S)$ then we have:

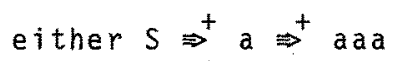

Now the first alternative can be discounted since it implies $L(F, \Rightarrow)$ is infinite. The second alternative can also be discounted since

$$
\text { a a } \Rightarrow^{m} \text { a }
$$

implies $a \stackrel{m}{\Rightarrow} \lambda$ and $a \stackrel{m}{\Rightarrow} a$ in which case

$$
\text { a a } \stackrel{m}{m} \text { a a }
$$

in $\mathrm{F}$.

Thus in all cases we are lead to a contradiction, hence $L$ is bad.

\section{III.4.3 The ETOL Situation}

Extending the notion of goodness and very completeness to ETOL forms, we compare the EOL and ETOL situations. The major difference is the role played by synchronization. This is because synchronization can occur in a different way than it does for EOL forms. Consider:

Lemma 4.10

The synchronized ETOL form $F=\left(\{S, a, N\},\{a\}, P_{1}, P_{2}, S\right)$ where:

$$
\begin{aligned}
& P_{1}: S \rightarrow S S ; a \rightarrow N ; N \rightarrow N \\
& P_{2}: S \rightarrow a ; a \rightarrow N ; N \rightarrow N
\end{aligned}
$$

generates no finite languages.

\section{Proof: Immediate.}

Synchronization occurs in $F$ because terminals are only introduced via $P_{2}$ and moreover any non-blocking word must give rise to terminals when $P_{2}$ is applied. Notice that $\mathcal{L}_{s}(F, \Rightarrow) \subseteq \mathcal{L}(E O L)$ and in particular:

$$
G: S \rightarrow S S ; S \rightarrow a ; a \rightarrow N ; N \rightarrow N
$$


has $L(G, \Rightarrow)=L(F, \Rightarrow)$. However $\mathscr{L}_{S}(G, \Rightarrow$ contains finite languages in contradistinction to $F$.

We now exhibit a synchronized ETOL form which is good. Again this is in contrast to the EOL situation.

\section{Theoxem 4.11}

Let $F$ be the two-tabled synchronized ETOL form defined by:

$$
\begin{aligned}
& P_{1}: S \rightarrow S ; a \rightarrow N ; N \rightarrow N \\
& P_{2}: S \rightarrow a ; a \rightarrow N ; N \rightarrow N .
\end{aligned}
$$

Then $F$ is good.

Proof: We proceed as in Theorem 4.2. Clearly $\mathcal{L}_{s}(F, \Rightarrow)=\mathcal{L}$ (ALPH). Let $\mathcal{L}(k)$ be the subfamily of $\mathcal{K}$ (ALPH) consisting of alphabets with at least $k$ symbols, $k>0$. Then $\mathscr{L}_{s}(\mathrm{~F}, \Rightarrow)=\mathcal{L}(1)$.

As in Theorem 4.2 the following claim can be established.

Claim: If $G$ is an ETOL form such that $\mathcal{L}_{s}(G, \Rightarrow \subseteq \mathcal{L}(A L P H)$ then $\mathcal{L}_{s}(G, \Rightarrow)=\mathcal{L}(k)$ for some $k>0$.

We now demonstrate that there are $F_{k} \rightarrow \mathrm{F}, k>0$ such that $\mathcal{L}_{s}\left(F_{k}, \Rightarrow\right)=\mathcal{L}(k)$.

$F_{1}$ is $F$ and for $k>1, F_{k}$ is defined by:

$$
\begin{aligned}
& P_{1}^{(k)}: s_{i} \rightarrow s_{i+1}, I \leq i<k ; s_{k} \rightarrow s_{k} ; a_{i} \rightarrow N, 1 \leq i \leq k ; N \rightarrow N \\
& p_{2}^{(k)}: s_{i} \rightarrow a_{i}, I \leq i \leq k ; a_{i} \rightarrow N, 1 \leq i \leq k ; N \rightarrow N .
\end{aligned}
$$

It should be clear that $\mathcal{L}_{\mathrm{s}}\left(\mathrm{F}_{\mathrm{k}}, \Rightarrow\right)=\mathcal{L}(\mathrm{k})$.

However the power of this different synchronization technique is 1 imited since:

\section{Theorem 4.12}

No synchronized ETOL form is EOL- or ETOL-vomplete.

Proof: We first prove:

Claim: Let $F: S \rightarrow a ; a \rightarrow a a$. Then no synchronized ETOL form is form equivalent to $F$. 
Proof of craim: Assume $G$ is an arbitrary synchronized ETOL form which is form equivalent to $F$. Then there is a $G^{\prime} \Delta, G$ such that $L(F, \Rightarrow)=$ $L\left(G^{\prime}, \Rightarrow\right)$. Let $G^{\prime}=\left(V,\{a\}, P_{1}, \ldots, P_{m}, S\right)$. Consider a derivation of $a^{4}$ in $G^{\prime}$,

$$
S \overrightarrow{\mathrm{P}}_{i_{1}} \alpha_{1} \Rightarrow_{i_{2}} \alpha_{2}{\overrightarrow{p_{i}}}_{i_{3}} \cdots \vec{p}_{i_{r}} \alpha_{r}=\text { aaa. }
$$

Since $G^{\prime}$ is synchronized the $\alpha_{i}$ are nonterminal words for $1 \leq i<r$. Now uniquely rename the symbols in the $\alpha_{i}, 1 \leq i \leq r$, such that $\alpha_{r}=$ bbbb, adding the new productions to the appropriate $\mathrm{P}_{i_{j}}$, and com-

pleting the other tables by adding productions in the following way.

If in $\alpha_{k} \Rightarrow \alpha_{k+1}$ using $P_{i_{k}}$ the production $A \rightarrow \beta$ is used then add a new production $A(k) \rightarrow \beta(k+1)$ to $P_{i_{k}}$, where the superscript indicates the renaming. Further add to all other tables $P_{\ell}, \ell \neq i_{k}$, the productions $A^{(k)} \rightarrow \gamma$ if $A \rightarrow \gamma$ is in $P_{\ell}$.

Let this new ETOL gramar be G". Clearly $G^{\prime \prime} \triangle_{S} G^{\prime}$ and further $L\left(G^{\prime \prime}, \Rightarrow\right)=\left\{a^{2^{n}}: n \geq 0\right\} \cup\{a b b b\}$. But this language is not obtainable via an interpretation of $F$, therefore $\mathcal{Z}_{S}(F, \Rightarrow) \neq \mathcal{Z}_{S}(G, \Rightarrow)$, a contradiction and the claim is established.

Returning to the theorem assume there is a synchronized ETOL form $H$ which is EOL-vomplete. Then there must be an $H^{\prime} \Delta_{S} H$ with $\mathscr{L}_{s}\left(H^{\prime}, \Rightarrow\right)=\mathcal{L}_{s}(F, \Rightarrow)$. This provides a contradiction. The case of ETOL-vompleteness is similar.

There are ETOL-complete forms which are good and some which are bad.

\section{Theorem 4.13}

Let $F$ be defined by:

and$$
P_{1}: S \rightarrow \lambda ; S \rightarrow a ; S \rightarrow S ; S \rightarrow S S ; a \rightarrow S
$$

$$
P_{2}: S \rightarrow S ; a+S
$$

then $F$ is good and ETOL-complete, while $G$ defined by:

and

$$
\mathrm{P}_{3}: S \rightarrow \mathrm{a} ; \mathrm{S} \rightarrow \mathrm{S} ; \mathrm{S} \rightarrow \mathrm{SS} ; \mathrm{a} \rightarrow \mathrm{N} ; \mathrm{N} \rightarrow \mathrm{N}
$$

$$
\mathrm{P}_{4}: \mathrm{S} \rightarrow \mathrm{S} ; \mathrm{a} \rightarrow \mathrm{N} ; \mathrm{N} \rightarrow \mathrm{N}
$$

is bad and ETOL-complete. 
Ppoof: The ETOL-completeness of $F$ and $G$ follows from Section 3.4 . That $G$ is bad follows directly from Theorem 4.12 while the goodness of $F$ follows from the binary normal form result for $E T O L$ forms in Section 3.4 .

We can now strengthen Theorem 4.6 considerably.

\section{Theorem 4.14}

Let $F$ be a propagating ETOL form. Then $F$ is neither ETOLvomplete nor EOL-vomplete.

Proof: This is established by first demonstrating:

Claim: Let $G$ be defined by the productions $S \rightarrow a b a ; b \rightarrow \lambda ; a \rightarrow c d$; $a \rightarrow c d ; c \rightarrow c ; d \rightarrow d$. Then there is no propagating ETOL form which is form equivalent to $G$.

Eroof of claim: Assume $H$ is such a propagating ETOL form. Then there is an $H^{\prime} \overleftrightarrow{s} H$ with $L\left(H^{\prime}, \Rightarrow\right)=L(G, \Rightarrow)$.

Case (i): Assume $H^{\prime}$ is synchronized. There are nt-derivations $S \Rightarrow^{+}$aba $\Rightarrow$ blocking and $S \Rightarrow+c d c d \Rightarrow+$ blocking since $H^{\prime}$ is synchronized. Consider an interpretation $H^{\prime \prime} \underset{5}{\Delta} H^{\prime}$ such that $L\left(H^{\prime \prime} \Rightarrow\right)=\{a b a, c d e f\}$. Again this is obtainable because of synchronization. But $L\left(H^{\prime \prime} \Rightarrow\right)$ is not obtainable via an interpretation of $G$, since $L\left(H^{\prime \prime}, \Rightarrow\right) \subseteq L\left(G^{\prime}, \Rightarrow\right)$ implies efcd, efef and cdcd are in $L\left(G^{\prime}, \Rightarrow\right)$. This provides a contradiction.

Case (ii): Assume $H^{\prime}$ is not synchronized. Then as in Theorem 4.6 we must have

$$
a b a \Rightarrow+c d c d
$$

and by a detailed case analysis, since $H^{\prime}$ is propagating, we obtain a contradiction.

The theorem now follows immediately. 


\section{III.4.4 Relative and Mutual Goodness}

Recall that an EOL form $F$ is good if for all EOL-grammatical

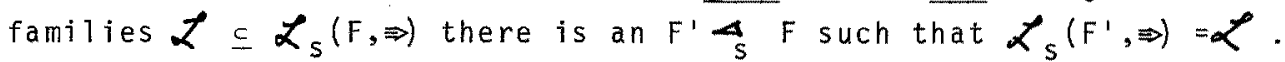
We extend this notion of goodness to: $F$ is good relative to a particular collection of EOL-grammatical families. Let $F$ and $G$ be two $E O L$ forms. We say that $F$ is good relative to $G$ ( $G$-good) if

$\mathcal{L}_{s}\left(G, \Rightarrow \subseteq \mathcal{L}_{s}(F, \Rightarrow)\right.$ and for all $G^{\prime} \rightarrow G$ there is an $F^{\prime} \rightarrow F_{s}$ such that $\mathcal{L}_{s}^{s}(F, \Rightarrow)=\mathcal{Z}_{s}^{s}\left(G^{\prime}, \Rightarrow\right)$. Analogously we say $F$ is $G$-bad if it is not G-good.

Thus for example, letting:

$$
\begin{aligned}
& F_{1}: S \rightarrow a ; a \rightarrow a \\
& F_{2}: S \rightarrow a ; a \rightarrow N ; N \rightarrow N \\
& F_{3}: S \rightarrow a ; a \rightarrow b ; b \rightarrow b
\end{aligned}
$$

then $F_{1}$ is both $F_{2}$-good and $F_{3}$-good, while $F_{2}$ is both $F_{1}$ - and $F_{3}$-bad and $F_{3}$ is also $F_{1}$ - and $F_{2}$-bad. These observations follow from the results in the previous sections.

That $F_{1}$ is $F_{3}$-good can be generalized as follows: Let $F, F^{\prime}$ be EOL forms with $F_{S} \rightarrow F$, then $F$ is $F^{\prime}$-good:

Similarly if $F, F^{\prime}$ and $G$ are three $E O L$ forms with $F^{\prime}{ }_{5} F$, then

$F^{\prime}$ is G-good implies $F$ is G-good.

Our first result on relative goodness is a transitivity

result.

\section{Lemma 4.15}

Let $F, G, G^{\prime}$ and $H$ be EOL forms with $G^{\prime} \underset{S}{A} G$. Then $F$ is G-good and $G^{\prime}$ is $H$-good implies $F$ is $H$-good.

Proof: Since $G^{\prime} \underset{S}{A} G, F$ is $G^{\prime}$-good and thus $F$ is $H$-good.

Relative goodness gives rise in a natural way to the mutual goodness of two EOL forms. We say two EOL forms $F$ and $G$ are mutually good if $F$ is G-good and $G$ is F-good.

Consider the following EOL forms:

For $n \geq 0$ :

$F_{n}: S_{i} \rightarrow S_{i+1}, 0 \leq i<n ; S_{n} \rightarrow a ; a \rightarrow N ; N \rightarrow N$.

It is clear that $\xi_{s}\left(F_{i}\right) \neq \xi_{s}\left(F_{j}\right)$ for all $i \neq j, i, j \geq 0$. However $\mathcal{L}_{s}\left(F_{j} \Rightarrow\right)=\mathcal{L}_{s}\left(F_{j} \Rightarrow\right.$ for all $i, j \geq 0$, by the simulation lemmas. Moreover it is not difficult to see that for all $F_{i} \Delta_{S} F_{j}$ there is an $F_{j}^{\prime} \uplus_{s} F_{j}$ such that $\mathcal{L}_{s}\left(F_{j}^{\prime}, \Rightarrow\right)=\mathscr{L}_{s}\left(F_{j}^{\prime}, \Rightarrow\right)$, for all $i, j \geq 0$. Thus $F_{i}$ is 
$F_{j}$-good and $F_{j}$ if $F_{i}$-good, that is $F_{i}$ and $F_{j}$ are mutually good for all $i, j \geq 0$. Notice that mutual goodness is an equivalence relation, hence we have:

Lemma 4.16

For two EOL forms $F$ and $G$ :

(i) $\xi_{s}(F)=\xi_{s}(G)$ implies $F$ and $G$ are mutually good but not vice versa.

(ii) $F$ and $G$ are mutually good implies $\mathcal{L}_{s}(F, \Rightarrow)=\mathcal{L}_{s}(G, \Rightarrow)$, but not vice versa.

Proof: The positive statements follow from the definitions. $F_{0}$ and $F_{1}$ as defined above yields the second half of $(i)$, while $S \rightarrow a ; a \rightarrow a$ and $F_{0}$ yields the second half of (ii).

Thus mutual goodness lies between strong form equivalence and form equivalence in its classification power. At the time of writing the decidability of mutual goodness remains an open problem.

We now present our main result on mutual goodness, namely "right linear" and "left linear" EOL forms are, surprisingly, not mutually good.

We say an EOL form $F$ is left linear if its nonterminal productions are left linear in the context-free grammar sense and its terminal productions are all of the type: $a \rightarrow b$, a and $b$ both terminal. Right linear EOL forms are similarly defined.

\section{Theorem 4.1 ?}

Let $F$ be a right linear EOL form defined by the productions $S \rightarrow a ; S \rightarrow b S ; a \rightarrow a ; b \rightarrow c ; c \rightarrow a$.

Then there is no left linear EOL form $H$ which is form equivalent to $F$.

Proof: First note that $L\left(F, \Rightarrow=a^{+} u a^{*} C a\right.$. Assume such an $H$ exists.

Then there is an $H^{\prime} \underset{5}{4} H$ such that $L\left(H^{\prime}, \Rightarrow\right)=L(F, \Rightarrow)$. Let $H^{\prime}=$ $\left(V, \Sigma, P, S^{\prime}\right)$, where $\{a, c\} \subseteq \Sigma$. Now since $F$ is deterministic on terminals, for any interpretation $F^{\prime} \vec{S}_{S} F(\mu)$, for each word $y$ in $L\left(F^{\prime}, \Rightarrow\right.$ such that $\mu^{-1}(y)=a^{i+2}$, for some $i \geq 0$, there is a word $x$ in $L\left(F^{\prime}, \Rightarrow\right.$ with $\mu^{-1}(x)=a^{i} c a$. Therefore there is terminal forcing present, since the derivation of $a^{i}$ ca cannot be isolated from that of $a^{i+2}$ in $H^{3}$. 0therwise this would immediately imply that $\mathcal{L}_{\mathrm{s}}(\mathrm{H}, \Rightarrow) \neq \mathcal{L}_{\mathrm{s}}(\mathrm{F}, \Rightarrow)$. 
Because the derivations of $a^{i} c a$ and $a^{i+2}$ are tied together in $H^{\prime}$, we have two possibilities:

(i) $a^{i+2} \Rightarrow a^{i} c a$, for some $m>0$, or

(ii) $a^{i} c a \Rightarrow a^{i+2}$, for some $m>0$.

Now (i) implies $a \Rightarrow c$, therefore since a is in $L(F, \Rightarrow)$, c must be in $L(F, \Rightarrow)$. A contradiction.

On the other hand (ii) implies $a \Rightarrow m$ and $c \Rightarrow a$, by the definition of left linear forms. 'Since $H^{\prime}$ is left linear the arrangement to deposit a " $c$ " in the penultimate position of a word a ${ }^{i}$ ca must be made at the second derivation step (terminals are deposited in a right-to-left manner with a left linear grammar). This, in turn, implies:

there is a terminal symbol d such that

$d \Rightarrow^{+} d$ and $d \stackrel{+}{+} c$.

If $d \neq a$ and $d \neq c$ then

$S^{\prime} \Rightarrow a^{i} d a$ in $H^{\prime}$,

a contradiction and if $d=a$, then $S \Rightarrow a \Rightarrow^{+} c$ in $H^{\prime}$, a contradiction. Finally if $d=c$, then consider an interpretation $H^{\prime \prime}$ of $H^{\prime}$ in which $d \Rightarrow+$ is not possible. Then $L\left(H^{\prime \prime}, \Rightarrow\right)$ is not in $\not{L}_{s}(F, \Rightarrow)$.

Thus $H$ does not exist and the theorem is proved.

\section{Corozzary 4.18}

Let $G$ be defined by the productions:

$\mathrm{S} \rightarrow \mathrm{a} ; \mathrm{S} \rightarrow \mathrm{aS} ; \mathrm{a} \rightarrow \mathrm{a}$

and $H$ by the productions:

$S \rightarrow a ; S \rightarrow S a ; a \rightarrow a$.

Then $G$ and $H$ are not mutually good.

Proof: Assume otherwise. Now if $G$ and $H$ are mutually good, then $H$ is G-good, but $F$ of Theorem 4.17, which is an interpretation of $G$, is not form equivalent to any interpretation of $H$. 


\section{III.5 Decidability Questions}

We consider some decidability issues in this section. The first which could as well be in chapter II concerns strong form equivalence. We not only show that strong form equivalence is decidable but also show that it is NP-complete. Second, in Section 5.2, the notion of uniform interpretations of $E O L$ forms is introduced and it is shown that in this case form equivalence is undecidable. For s-interpretations this is still an open problem, while for ginterpretations of grammar forms it has recently been shown to be decidable. Finally we discuss $O L$ forms and demonstrate that form equivalence is decidable for POL forms, while form equivalence is undecidable for $0 L$ forms under full interpretation. We omit any discussion of form equivalence for PDOL forms under deterministic interpretation. Surprisingly form equivalence has in this case also been shown to be decidable.

\section{III.5.1 Strong Form Equivalence}

Recall that two EOL forms $F_{1}$ and $F_{2}$ are strong form equivalent if $\xi_{s}\left(F_{1}\right)=\xi_{s}\left(F_{2}\right)$. Now recall also that $g_{s}\left(F_{1}\right)=g_{s}\left(F_{2}\right)$ iff $F_{1} \underset{s}{\Delta} F_{2}$ and $F_{2} \Delta F_{1}$. Thus the decidability of strong form equivalence hinges on the decidability of $\Delta_{s}$. We now prove that $\vec{s}$ is decidable.

\section{Theorem 5.1}

$\underset{s}{\otimes}$ is decidable for EOL forms.

Proof: Let $F_{i}=\left(V_{i}, \Sigma_{j}, P_{i}, S_{i}\right) i=1,2$ be two arbitrary $E O L$ forms. We wish to demonstrate that it is decidable whether or not there exists a dfl-substitution $\mu$ such that $F_{1} \underset{s}{4} F_{2}(\mu)$.

Consider the collection $M$, of a 11 dfl-substitutions $\mu: V_{2}^{*} \rightarrow 2_{1}^{*}$ satisfying the further properties

(i) $\mu(a) \subseteq \Sigma_{1}$, for al1 a in $\Sigma_{2}$,

(ii) $\mu(A) \subseteq V_{1}-\Sigma_{1}$, for al1 A in $V_{2}-\Sigma_{2}$, and (iii) $s_{1}$ is in $\mu\left(s_{2}\right)$.

clearly $M$ is finite. Now $F_{1} \underset{s}{\Delta} F_{2}$ iff there is a $\mu$ in $M$ satisfying $P_{1} \subseteq \mu\left(P_{2}\right)$. Clearly this is decidable, hence the result. 
It should be clear that we have really not used any EOLproperties of $\mathrm{F}_{1}$ and $\mathrm{F}_{2}$ in the proof of Theorem 5.1, hence:

\section{Corolzary 5.2}

$\underset{s}{\rightarrow}$ is decidable for grammar forms.

The situation for $\vec{g}$ is only sightly more complex. Let $M$ be the collection of all finite substitutions $\mu: V_{2}^{*} \rightarrow 2_{1}^{*}$ satisfying:

(i) $\mu$ is a dfl-substitution on $v_{2}-\Sigma_{2}$,

(ii) $\mu(a)=\left\{x: x\right.$ is in $\Sigma_{\uparrow}^{*}$ and there is a production

$A \rightarrow \alpha \times \beta$ in $\left.P_{1}\right\}$, for all a in $\Sigma_{2}$,

(iii) $\mu(A) \subseteq V_{1}-\Sigma_{1}$, for al1 $A$ in $V_{2}-\Sigma_{2}$, and

(iv) $S_{1}$ is in $\mu\left(S_{2}\right)$.

once again $M$ is finite and $F_{1} \underset{g}{\triangle} F_{2}$ iff there is a $\mu$ in $M$ such that $P_{1} \subseteq \mu\left(P_{2}\right)$. We have shown

\section{Theorem 5.3}

$\underset{g}{4}$ is decidable for grammar forms.

In general for any interpretation $\underset{x}{\vec{x}}$, we have that $\vec{x}$ is decidable, since we only use the finiteness of the collection of possible finite substitutions.

We now show that $\rightarrow$ is, in fact, NP-complete by showing that it is reducibly-equivalent to the clique problem which is a well known NP-complete problem.

\section{Theorem 5.4}

The decidability of $\underset{s}{a}$ and $\underset{g}{a}$ are NP-complete problems.

Proof: We will prove that the clique problem for graphs can be solved in polynomial time iff the decidability of $\underset{s}{\Delta}$ can be solved in polynomial time.

Note that we define a graph to be directed, without multiple edges and without self-loops. Let $H=(N, E)$ be a directed graph where $N$ is the finite nonempty set of vertices and $E \subseteq N \times N$ is the set of directed edges. Since $H$ has no self-loops, ( $u, u)$ is not in $E$ for any $u$ in $N$. We say $H$ has a clique if there is some subset $M \subseteq N$ of vertices such that $(u, v)$ and $(v, u)$ are in $E$ for all $u, v$ in $M$ with $u \neq v$.

We encode $H$ as a grammar $G_{H}$ as follows: 
(i) $G_{H}=\left(V, \Sigma, P_{H}, S_{H}\right)$,

(ii) $V=\{[u]: u$ is in $N\} \cup\left\{S_{H}\right\}$ and $\Sigma=\emptyset$, and

(iij) $p=\left\{S_{H} \rightarrow[u],[u] \rightarrow \lambda: u\right.$ is in $\left.N\right\}$ u $\{[u] \rightarrow[v]:(u, v)$ is in $E\}$.

clearly $G_{H}$ can be obtained from $H$ in polynomial time.

In a similar manner every grammar which is similar to $G_{H}$, that

is an s-interpretation of: $S \rightarrow A ; A \rightarrow A ; A \rightarrow d$; can be encoded as a directed graph. Again this is a polynomial time encoding.

Let $H^{\prime}=\left(N^{\prime}, E^{\prime}\right)$ be a directed graph with $N^{\prime}=m \geq 1$ and $E^{\prime}=N^{\prime} \times N^{\prime}-\left\{\left(u^{\prime}, u^{\prime}\right): u^{\prime}\right.$ in $\left.N^{\prime}\right\}$, that is $H^{\prime}$ is the complete directed graph on m nodes. Let $H=(N, E)$ be an arbitrary directed graph of $n$ nodes.

Claim: $G_{H}, \Delta_{s} G_{H}(\mu)$ iff $H$ has a clique of size m.

Proof of claim: First assume $G_{H} \underset{s}{\Delta} G_{H}(\mu)$ for some dfl-substitution $\mu$.

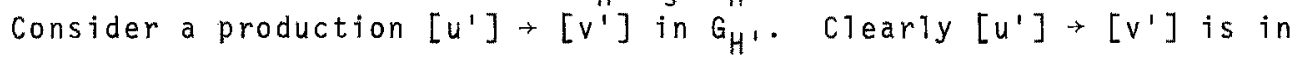
$\mu([u] \rightarrow[v])$ for some $[u] \rightarrow[v]$ in $G_{H}$. We show that this is the only production in $\mu([u] \rightarrow[v])$.

For assume otherwise, that is it also contains another production $\left[w^{\prime}\right] \rightarrow\left[x^{\prime}\right]$, then we have $\left[u^{\prime}\right] \neq\left[w^{\prime}\right],\left[v^{\prime}\right] \neq\left[x^{\prime}\right]$ or both. Without loss of generality assume $\left[u^{\prime}\right] \neq\left[W^{\prime}\right]$. In this case $\mu\left([u] \supseteq\left\{\left[u^{\prime}\right],\left[W^{\prime}\right]\right\}\right.$ and because $H^{\prime}$ is complete, $\left(u^{\prime}, w^{\prime}\right)$ is in $E^{\prime}$. But this implies $(u, u)$ is in E, a contradiction.

Thus we have shown that whenever $\left[u^{\prime}\right] \rightarrow\left[v^{\prime}\right]$ is in $\mu([u] \rightarrow[v])$ for some [u] and [v] then it is the only such production, hence $\mu^{-1}$ is an injection and therefore $H$ has a clique of size $\mathrm{m}$.

Conversely assume $H$ has a $c l i q u e$ of size $m$, then it is straightforward to define $\mu$ such that $G_{H}, \underset{s}{\Delta} G_{H}(\mu)$. This completes the proof of the claim.

Returning to the theorem, we have established that the clique problem is polynomial equivalent to the s-interpretation problem for a subclass of the context-free grammars. Clearly the s-interpretation problem for the class of a 11 context-free grammars is no easier than for this subclass. Therefore as the clique problem is NP-complete so is the s-interpretation problem. 


\section{III.5.2 Uniform Interpretations of EOL Forms}

In logic it is usual to consider substitutions which are applied uniformly, that is each appearance of a particular letter is replaced in the same way. Similarly when considering EOL forms we introduce uniform substitutions on the terminals, which enables interpretation grammars to maintain a tighter similarity to the original form than under the usual interpretation mechanism. For example given the production

(*) $\quad A \rightarrow a B a b$

then

$$
A \rightarrow a B b c
$$

is an s-interpretation of $(*)$ but not a uniform (on terminals) interpretation, white

$$
A \rightarrow b B b c
$$

is a uniform interpretation of $(*)$. Similarly given the production

$$
(* *) \quad a \rightarrow a B b
$$

then

$$
b \rightarrow b A d
$$

is a uniform interpretation of $(* *)$ while

$$
b \rightarrow c A d
$$

is not.

More formally, given two EOL forms $F_{i}=\left(V_{i}, \Sigma_{i}, P_{i}, S_{i j}\right) i=1,2$, we say that $F_{1}$ is a uniform interpretation (u-interpretation) of $F_{2}$ modulo $\mu$, denoted $F_{1} \leftrightarrow F_{2}(\mu)$ for some dfl-substitution $\mu$, if

(i) $F_{1} \rightarrow F_{2}(\mu)$, and

(ii) $P_{1} \subseteq \mu_{u}\left(P_{2}\right)$, where $\mu_{u}\left(P_{2}\right)=\underbrace{}_{x_{0} \rightarrow \alpha \text { in } P_{2}} \mu_{u}\left(x_{0} \rightarrow a\right)$ and

$$
\begin{aligned}
\mu_{u}\left(x_{0} \rightarrow \alpha\right)=\left\{x_{0}^{\prime} \rightarrow \alpha^{\prime}:\right. & x_{0}^{\prime} \rightarrow \alpha^{\prime} \text { is in } \mu\left(x_{0} \rightarrow \alpha\right), \alpha=x_{1} \ldots x_{m}, \\
& \alpha^{\prime}=x_{1}^{\prime} \ldots x_{m}^{\prime} \text { and for al1 i, } j, \\
& 0 \leq i \leq j \leq m, x_{i}, x_{j} \text { in } \Sigma_{2} \text { and } \\
& \left.x_{i}=x_{j} \text { implies } x_{i}=x_{j}\right\}
\end{aligned}
$$

We say $F_{1}$ is a full uniform interpretation (fu-interpretation) of $F_{2}$ modulo $\mu$, written $F_{1} \underset{\text { fu }}{\triangle} F_{2}(\mu)$ iff $F_{1} \underset{u}{\Delta} F_{2}(\mu)$ and $P_{1}=\mu_{u}\left(P_{2}\right)$.

We denote the language families of an EOL form $F$ under uniform and full uniform interpretation by $\mathcal{Z}_{u}(F, \Rightarrow)$ and $\mathcal{L}_{f u}(F, \Rightarrow$ respectively. clearly $\underset{u}{\Delta}$ and $\overrightarrow{f u}$ are decidable, by the techniques of section 5.1 , and moreover they are also preorders, cf. Section II.3.1. As an added attraction it is possible to exhibit EOL forms $F$ such that $\mathcal{Z}_{u}(F, \Rightarrow)=\mathcal{Z}(C F)$, which is not possible with s-interpretations. To demonstrate this fact we make use of the following proposition. 


\section{Proposition 5.5}

Let $F=(V, \Sigma, P, S)$ be an EOL grammar satisfying the condition that for $a 11$ a in $\Sigma$, a $\rightarrow$ a is the only production in $P$ for $a$.

Then $L(F \Rightarrow)$ is a context-free language.

When $F=(V, \Sigma, P, S)$, an EOL form, satisfies the condition of Proposition 5.5, we say $F$ is stable with respect to terminals. In this case let $G_{F}=\left(V, \Sigma, P_{F}, S\right)$ be the associated context-free grammar form obtained from $F$ by letting $P_{F}=P \cap\left((V-\Sigma) \times V^{*}\right)$.

We say an EOL form $F=(V, \Sigma, P, S)$ is t-separated if for all $\mathrm{A}$ in $V-\Sigma$ and $A \rightarrow \alpha$ in $P, \alpha=\alpha_{1} a \alpha_{2} b \alpha_{3}$, for $a, b$ in $\Sigma$, for some $\alpha_{1}, \alpha_{2}, \alpha_{3}$ in $V^{*}$ implies $a \neq b$.

We now have:

\section{Theorem 5.6}

Let $F$ be an EOL form which is both stable with respect to terminals and $t$-separated then $\mathscr{\chi}_{u}(F, \Rightarrow)=\mathscr{\mathcal { L }}_{\mathrm{s}}\left(G_{F}, \Rightarrow\right)$.

Proof: Consider $\mathcal{L}_{u}(F, \Rightarrow) \subseteq \mathcal{L}_{S}\left(G_{F}, \Rightarrow\right)$. The only productions for terminals in $F$ are $a \rightarrow$ a which remain stable under u-interpretations, hence $L\left(F^{\prime} \Rightarrow\right.$ is in $\mathcal{L}(C F)$ for each $F^{\prime} \leftrightarrow F$. Furthermore since $F$ is t-separated u-interpretation and s-interpretation coincide for the nonterminal productions. Conversely, consider $\mathcal{Z}_{\mathrm{S}}\left(G_{F}, \Rightarrow\right) \subseteq \mathcal{Z}_{u}(F, \Rightarrow)$. For each $G_{F}^{i} \underset{S}{\Delta} G_{F}$ we can construct $F^{\prime} \underset{u}{\Delta} F$ with $L\left(G_{F}^{\prime}, \Rightarrow\right)=L\left(F^{\prime}, \Rightarrow\right.$ by adding stable productions for terminals, applying proposition 5.5 and using the fact that $F$ and hence $G_{F}$ are $t$-separated.

In the case that $F$ is not $t$-separated we have

\section{Corozzary 5.7}

Let $F$ be an EOL form stable with respect to terminals. Then $\mathcal{L}_{u}(F, \Rightarrow) \subseteq \mathcal{L}_{s}\left(G_{F}, \Rightarrow\right)$ and $\not{\swarrow}_{u}(F, \Rightarrow \subseteq \mathcal{X}(C F)$.

However applying Theorem 5.6 directly we obtain:

Corozzary 5.8

$$
\mathrm{F}_{1}: \mathrm{S} \rightarrow \mathrm{SS} ; \mathrm{S} \rightarrow \mathrm{a} ; \mathrm{a} \rightarrow \mathrm{a}
$$

and

$$
F_{2}: S \rightarrow a S S ; S \rightarrow a S ; S \rightarrow a ; a \rightarrow a
$$

are context-free complete under u-interpretation. 
The characterization of $\mathcal{L}_{u}(\mathrm{~F}, \Rightarrow)$ in terms of the associated grammatical family $\mathcal{L}_{S}\left(G_{F}, \Rightarrow\right)$ given in Theorem 5.6 leads to consideration of a weaker notion of $u$-interpretation.

Given two $E O L$ forms $F_{i}=\left(V_{i}, \Sigma_{i}, P_{i}, S_{i}\right), i=1,2$ we say that $F_{1}$ is a weak u-interpretation (wu-interpretation) of $F_{2}$ modulo $\mu$, denoted $F_{1} \underset{w u}{\Delta} F_{2}$, for some dfl-substitution $\mu$, if

(i) $F_{1} \underset{s}{\rightarrow} F_{2}(\mu)$, and

(ii) $\mathrm{P}_{1} \subseteq \mu_{w u}\left(\mathrm{P}_{2}\right)$, where

$$
\mu_{w u}\left(x_{0} \rightarrow x_{1} \ldots x_{m}\right)=\left\{\begin{aligned}
& \mu\left(x_{0}\right.\left.\rightarrow x_{1} \ldots x_{m}\right) \text { if } x_{0} \text { is in } v_{2}-\Sigma_{2} \text {, and } \\
&\left\{x_{0}^{1}\right. \rightarrow x_{1}^{1} \ldots x_{m}^{1} \text { : where } x_{i}=x_{0} \text { implies } \\
&\left.x_{1}^{\prime}=x_{0}^{1} \text { for all i, } 1 \leq i \leq m\right\} \\
& \text { if } x_{0} \text { is in } \Sigma_{2} .
\end{aligned}\right.
$$

As before we also speak of full wu-interpretation, denoted $F_{1} \underset{f w u}{\Delta} F_{2}$. observe that $\mathcal{Z}_{u}\left(F, \Rightarrow \subseteq \mathcal{Z}_{w u}(F, \Rightarrow)\right.$ and $\mathcal{Z}_{f u}\left(F, \Rightarrow \subseteq \mathcal{Z}_{f w u}(F, \Rightarrow)\right.$ for all EOL forms $F$. That these inclusions can be proper is seen by considering $F: S \rightarrow a b b ; a \rightarrow a ; b \rightarrow b$. Clearly for $F^{\prime}: S \rightarrow a b c ; a \rightarrow a$; $b \rightarrow b ; c \rightarrow c$, we have $F^{\prime} \underset{w u}{ } F$ but $F^{\prime}$ is not a u-interpretation of $F$. However, every wu-family is a u-family since we can always ensure that $F$ is t-separated without disturbing the wu-family. This also holds for s-families, since in this case we consider s-form equivalent separated forms. Immediately every s-family is a wu-family and a u-family. We can now modify Theorem 5.6 as follows:

\section{Theorem 5.9}

Let $F$ be an EOL form stable with respect to terminals. Then $\mathcal{L}_{W U}(F, \Rightarrow)=\mathcal{L}_{S}\left(G_{F}, \Rightarrow\right)$.

Proof: As in Theorem 5.6 observing that wu-interpretation preserves stability.

In the next section we will consider the undecidability of the equation $\mathscr{L}_{f u}\left(F_{1} \Rightarrow\right)=\mathcal{L}_{f u}\left(F_{2}, \Rightarrow\right.$ for arbitrary $E O L$ forms $F_{1}$ and $F_{2}$. Here we show that the equation $\mathcal{L}_{f u}\left(F_{1}, \Rightarrow\right)=\mathcal{L}_{f w u}\left(F_{2}, \Rightarrow\right)$ is undecidable for arbitrary $F_{1}$ and $F_{2}$ by showing that $\mathcal{L}_{f u}(F, \Rightarrow)=\mathcal{Z}_{f w u}(F, \Rightarrow$ is undecidable for arbitrary $F$. 


\section{Theorem 5.10}

For arbitrary EOL forms $F$ it is undecidable whether

$\mathcal{L}_{f u}(F, \Rightarrow)=\mathcal{X}_{f w u}(F, \Rightarrow)$

Proof: We reduce the above question to the Post correspondence Problem $(P C P)$. Consider an arbitrary instance of the PCP

$$
\left(x_{1}, \ldots, x_{n}\right)\left(y_{1}, \ldots, y_{n}\right) \text {, }
$$

where $x_{i}, y_{i}$ are nonempty words over the alphabet $\left\{_{1}, a_{2}\right\}$. We define two encodings of $\left\{a_{1}, a_{2}\right.$, \# $\}$ by the homomorphisms $g$ and $h$ :

$$
\begin{aligned}
& g(\#)=Y X^{2} Y ; g\left(a_{1}\right)=Y X^{3} Y ; g\left(a_{2}\right)=Y X^{4} Y ; \\
& h(\#)=b a^{2} b ; h\left(a_{1}\right)=b a^{3} b ; h\left(a_{2}\right)=b a^{4} b .
\end{aligned}
$$

Consider the following EOL grammar $F=(V, \Sigma, P, S)$ where $V=\Sigma u\left\{S, S_{1}, S_{2}, S_{3}, S_{4}, S_{5}, X, Y\right\}, \Sigma=\{a, b\}$ and $P$ consists of the following two groups of productions.

$$
\left\{\begin{array}{l}
s \rightarrow s_{1} ; \\
s_{1} \rightarrow c s_{1} c ; s_{1} \rightarrow g\left(a_{1}\right) s_{2} g\left(a_{2}\right) ; s_{1} \rightarrow g\left(a_{2}\right) s_{2}\left(a_{1}\right) \\
s_{1} \rightarrow c s_{3} ; s_{1} \rightarrow s_{4} c ; \\
s_{2} \rightarrow c s_{2} ; s_{2} \rightarrow s_{2} c ; s_{2} \rightarrow g(\#) ; \\
s_{3}+c s_{3} ; s_{3} \rightarrow g(\#) ; \\
s_{4} \rightarrow s_{4} c ; s_{4} \rightarrow g(\#) ; \\
x \rightarrow a ; y \rightarrow b ; a \rightarrow a ; b \rightarrow b ;
\end{array}\right.
$$

where $c=g\left(a_{1}\right)$ or $g\left(a_{2}\right)$.

$$
\left\{\begin{array}{l}
s_{\rightarrow} \rightarrow h\left(x_{i}\right) s_{5} m i\left(h\left(y_{i}\right)\right) ; \\
s_{5} \rightarrow h\left(x_{i}\right) s_{5} m i\left(h\left(y_{i}\right)\right) ; \\
s_{5} \rightarrow h(\#)
\end{array}\right\} \text { for all } i, 1 \leq i \leq n
$$

Now the group (1) productions generate all coded versions of words of the type $x \# y$ where $x, y$ are over $\left\{a_{1}, a_{2}\right\}$ and $x \neq m i(y)$. Moreover for these productions u-interpretations and wu-interpretations have the same effect since the nonterminal productions are $t$-separated and the terminal productions are stable. This is not true for the group (2) productions. The group (2) productions generate an encoded version of a word of the type $x \# m i(x)$ exactly in the case that the PCP has a solution. It is now sufficient to establish the following claim.

Claim: $\mathscr{L}_{f u}\left(F, \Rightarrow=\mathscr{L}_{f w u}(F, \Rightarrow)\right.$ iff the given instance of the PCP has no solution. 
Proof of Claim: Assume that the PCP has a solution. Then $\mathcal{L}_{\text {fwu }}(F, \Rightarrow)$ contains a language containing a word $x h(\#) m i(x)$ where $x=b a_{1} a_{2} \ldots b b a_{1} a_{2} \ldots b . .$. , that is the a's between the b's are different. Such a word is generated by interpretations of the productions in the second group, by interpreting $b$ as $b$ and $a$ as $\left\{a_{1}, a_{2}, a_{3}, a_{4}\right\}$. By our observations above no such word belongs to any language in $\mathcal{L}_{f u}(F, \Rightarrow)$. For, if it did, then it would have been generated exclusively by the productions in the first group, since u-interpretations of group (2) productions cannot give rise to different a's between the $b^{\prime} s$. Now if $b$ is only interpreted as $b$, group (1) productions cannot give rise to such a word. Therefore assume some of the $a_{i}$ 's are also interpretations of the $b$. Now observe

(i) between any two interpretations of b zero, two, three or four interpretations of a occur, and

(ii) three consecutive interpretations of b never occur.

Under fu-interpretation if any one of the $a_{i}$ 's is an interpretation of $b$ then at least one of ( $i$ ) and ( $i i)$ are contradicted.

We have shown that if the given instance of the PCP has a solution, then $\mathcal{L}_{f u}(F, \Rightarrow) \neq \mathcal{L}_{f w u}(F, \Rightarrow)$.

Secondly assume the given instance of the PCP has no solution. Then any word in any language in $\mathcal{L}_{f w u}(F, \Rightarrow)$ or $\mathcal{L}_{f u}(F, \Rightarrow)$ can be generated exclusively using group (1) productions. Hence the claim has been established and also the Theorem.

The claim which has been proved in the above Theorem is no longer valid if $u$ - and wu-interpretations are considered. In fact, if $n=1, x_{1}=$ aa and $y_{1}=$ a then $\mathcal{L}_{u}\left(F, \Rightarrow \subsetneq \mathcal{L}_{w u}(F, \Rightarrow)\right.$ since

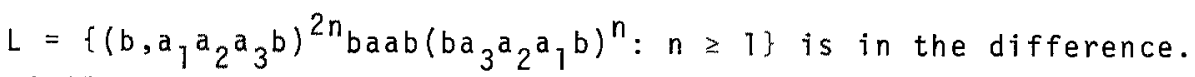
Similar difficulties are met if an attempt is made to show that $\mathcal{L}_{x}\left(F_{1}, \Rightarrow\right)=\mathcal{Z}_{x}\left(F_{2} \Rightarrow\right)$ is undecidab $)$ e for $x=u$ or wu. Even under s-interpretations this question is open.

However we are able to show that the emptiness of intersection of $\mathcal{L}_{u}\left(F_{1}, \Rightarrow\right.$ and $\mathcal{L}_{u}\left(F_{2}, \Rightarrow\right.$ is undecidable.

\section{Theorem 5.11}

The emptiness of $\mathcal{L}_{u}\left(F_{1}, \Rightarrow\right)_{n} \mathcal{L}_{u}\left(F_{2}, \Rightarrow\right)$ is undecidable for arbitrary EOL forms $F_{1}$ and $F_{2}$. 
Eroof: We actually prove a stronger result, namely, the emptiness of intersection is undecidable for EOL forms stable with respect to terminals.

Let $\left(x_{1}, \ldots, x_{n}\right)$ and $\left(y_{1}, \ldots, y_{n}\right)$ be an arbitrary instance of the PCP where $n \geq 1$ and $x_{i}, y_{i}$ are nonempty words over $\left\{a_{1}, a_{2}\right\}$-for all $i, 1 \leq i \leq n$. Define a homomorphism $h$ on $\left\{\#, a_{1}, a_{2}, 1, \ldots, n\right\}$ by:

$$
\begin{aligned}
& h(H)=b a^{2} b ; h\left(a_{1}\right)=b a^{3} b ; h\left(a_{2}\right)=b a^{4} b ; \\
& h(i)=b a^{4+i_{b}}, 1 \leq i \leq n .
\end{aligned}
$$

Let $F_{1}$ and $F_{2}$ be two EOL forms defined by the following sets of productions:

and

$$
F_{1}: S \rightarrow h(i) S h\left(x_{i}\right) ; S \rightarrow h\left(i \# x_{i}\right) ; \text { for } 1 \leq i \leq n ; a \rightarrow a ; b \rightarrow b
$$

$$
F_{2}: S \rightarrow h(i) S h\left(y_{i}\right) ; S \rightarrow h\left(i \# y_{i}\right) ; \text { for } 1 \leq i \leq n ; a \rightarrow a ; b \rightarrow b .
$$

We now claim that

Craim: $\mathcal{L}_{u}\left(F_{1}, \Rightarrow\right) \mathcal{L}_{u}\left(F_{2}, \Rightarrow\right)=\emptyset$ iff the PCP has no solution.

Proof of Claim: Assume the PCP has no solution. Consider an arbitrary word $x$ in a language of $F_{1}$. We can write $x$ as:

$$
\beta_{i_{1}} \cdots \beta_{i_{m}}^{\gamma \alpha_{i}} \cdots^{\alpha_{i}}
$$

where the $\beta_{i_{j}}$ are interpretations of $h\left(i_{j}\right), \gamma$ of $h(\#)$ and the $\alpha_{i_{j}}$ of $h\left(x_{i_{j}}\right)$. This decoding is unique because the u-interpretation forces each $\beta_{f_{j}}$ to be of the form $b_{j} a_{j}{ }_{j_{b}}$ and the corresponding $\alpha_{i j}$ to have blocks of $b a^{3} b$ and $b a^{4} b$ replaced by $b_{j} a_{j}^{3} b_{j}$ and $b_{j} a_{j}^{4} b_{j}$. This also hoids for the centre marker as well. Now if $x$ would also be a word in some language of $F_{2}$, then $x$ could be uniquely decoded as $\beta_{i_{1}} \ldots \beta_{i_{m}} \gamma \bar{\alpha}_{i_{m}} \ldots \bar{\alpha}_{i_{1}}$ where the $\bar{\alpha}_{i_{j}}$ are interpretations of $h\left(y_{i_{j}}\right)$. But this would imply the PCP has a solution, contradicting the assumption.

Now assume the PCP has some solution $x_{i_{m}} \ldots x_{i_{i}}=y_{i_{m}} \ldots y_{i_{i}}$.

We can construct an $\mathrm{F}_{1} \vec{u}_{1}$ with the productions:

$$
\begin{aligned}
& s \rightarrow h\left(i_{1}\right) s_{1} h\left(x_{i_{1}}\right) ; \\
& s_{m-1} \rightarrow h\left(i_{m}\right) \operatorname{sh}\left(x_{i_{m}}\right) ; s_{m-1} \rightarrow h\left(i_{m} \# x_{i_{m}}\right) ; \\
& a \rightarrow a ; b \rightarrow b
\end{aligned}
$$

such that $L\left(F_{j}, \Rightarrow\right)=\left\{h\left(\left(i_{1} \ldots i_{m}\right)^{t} \#\left(x_{i_{m}} \ldots x_{i_{1}}\right)^{t}\right): t \geq 1\right\}$.

Similarly we can construct $F_{2}^{\prime} \Delta F_{2}$ such that $L\left(F_{2}^{\prime}, \Rightarrow\right)=L\left(F_{1}^{\prime}, \Rightarrow\right)$. Thus $\mathcal{X}_{u}\left(F_{1}, \Rightarrow \cap \mathcal{L}_{u}\left(F_{2}, \Rightarrow\right) \neq 0\right.$. 
We have established both the claim and the Theorem.

Whether the above Theorem remains valid when $u$ is replaced by wh is stilt open.

\section{III.5.3 OL Forms and Form Equivalence}

In this section after introducing $0 \mathrm{~L}$ forms we prove two results. First, given two arbitrary $O L$ forms $F_{1}$ and $F_{2}$ it is undecidable whether $\mathcal{Z}_{f s}\left(F_{1} \Rightarrow\right)=Z_{f s}\left(F_{2}, \Rightarrow\right)$, and second given two POL forms $F_{1}$ and $F_{2}$ one of which has a single letter alphabet it is decidable whether $\mathcal{Z}_{s}\left(F_{1}, \Rightarrow\right)=\mathcal{Z}_{s}\left(F_{2}, \Rightarrow\right)$.

The former result also implies that given two arbitrary EOL forms $F_{1}$ and $F_{2}$ it is undecidable whether $\mathcal{L}_{f s}\left(F_{1}, \Rightarrow\right)=\mathcal{L}_{f s}\left(F_{2}, \Rightarrow\right)$, whether $\mathcal{L}_{f u}\left(F_{1}, \Rightarrow\right)=\mathcal{L}_{f u}\left(F_{2}, \Rightarrow\right)$ or whether $\mathcal{L}_{f w u}\left(F_{1}, \Rightarrow\right)=\mathcal{L}_{f w u}\left(F_{2}, \Rightarrow\right)$. An $0 L$ grammar $F=(\Sigma, P, \sigma)$ has $\sigma$ in $\Sigma^{+}$and $P$ in $\Sigma \times \Sigma^{*}$, where $P$ is a finite set of productions such that there is at least one production in $P$ for each a in $\Sigma$. We say $F$ is propagating, a POL grammar, if $a \rightarrow \lambda$ is not in $P$ for any a in $\Sigma$.

We say an $O L$ grammar $F=(\Sigma, P, \sigma)$ is reduced if for all a in $\Sigma$ there is a word $x$ in $\Sigma^{*}\{a\} \Sigma^{*}$ such that $\sigma \Rightarrow^{*} x$.

An $O L$ form is a reduced $O L$ grammar. Given two $O L$ forms $F_{i}=\left(\Sigma_{i}, P_{i}, \sigma_{j}\right) i=1,2$ and letting $\mu$ be a dfl-substitution on $\Sigma_{2}$, we say $F_{1}$ is an interpretation of $F_{2}$ modulo $\mu, F_{1} \triangleleft_{s} F_{2}(\mu)$ if (i) $\sigma_{1}$ is in $\mu\left(\sigma_{2}\right)$ and $(i i) P_{1} \subseteq \mu\left(P_{2}\right)$. Similarly we define $F_{1} f_{s} F_{2}(\mu)$ if $F_{1} \& F_{2}(\mu)$ and $P_{1}=\mu\left(P_{2}\right)$.

Clearly all other notions carry over to this situation.

To prove our first result we proceed via the following technical lemma.

\section{Lemma 5.12}

Let $F_{i}=\left(\Sigma_{i}, P_{i}, \sigma_{i}\right) i=1,2$ be two 0 L forms such that $F_{1} \underset{f s}{A} F_{2}(\mu)$. Then $L\left(F_{1}, \Rightarrow\right)=\left\{\sigma_{1}\right\} \underbrace{\bigcup_{\mu}}_{\sigma_{2} \Rightarrow^{+} x \text { in } F_{2}} \mu(x)$, where $\sigma_{1}$ is in $\mu\left(\sigma_{2}\right)$.

Proof: Follows directly from the definitions, noting that $\mu\left(x_{1} a x_{2}\right)=\emptyset$ if $\mu(a)=\emptyset$. 
This technical lemma tells us that the language of an fsinterpretation, given $\mu$, is completely determined by the axiom.

We now characterize the equality of two $0 \mathrm{~L}$ form families under fs-interpretations in terms of the lan uages of the given OL forms.

\section{Theorem 5.13}

Let $F_{i}=\left(\Sigma_{i}, P_{i}, \sigma_{i}\right)^{i}=1,2$ be two 0 L forms.

Then $\mathcal{Z}_{f s}\left(F_{1} \Rightarrow\right)=\mathcal{Z}_{f s}\left(F_{2}, \Rightarrow\right.$ iff there are $F_{1} \triangleleft F_{1}\left(\mu_{1}\right)$ and $F_{2}^{\prime} \underset{f s}{\Delta} F_{2}\left(\mu_{2}\right)$ such that $L\left(F_{1}^{\prime} \Rightarrow\right)=L\left(F_{2}, \Rightarrow\right.$ and $L\left(F_{2}^{1}, \Rightarrow\right)=L\left(F_{1} \Rightarrow\right)$.

Eroof: Assume $\mathcal{L}_{\mathrm{fs}}\left(\mathrm{F}_{1}, \Rightarrow\right)=\mathcal{L}_{\mathrm{fs}}\left(\mathrm{F}_{2}, \Rightarrow\right)$ then the condition is surely fulfilled.

Conversely assume there exist $F_{1}^{\prime}$ and $F_{2}^{\prime}$ as specified in the theorem statement. Consider an arbitrary $F_{1}^{\prime \prime} F_{s} F_{1}(\mu)$. Then $L\left(F_{1}^{\prime \prime} \Rightarrow\right)=\left\{\sigma_{1}^{\prime}\right\} \cup \underbrace{}_{\sigma_{1} \Rightarrow^{t} x \text { in } F_{1}} \mu(x)$ where $\sigma_{1}^{\prime \prime}$ is in $\mu\left(\sigma_{1}\right)$, by Lemma 5.12 .

However by assumption

$L\left(F_{1}, \Rightarrow\right)=\left\{\sigma_{1}\right\}$ u $\underbrace{}_{\sigma_{2} \Rightarrow y \text { in } F_{2}} \mu_{2}(y)$ where $\sigma_{1}$ is in $\mu_{2}\left(\sigma_{2}\right)$.

Combining these two expressions we obtain:

$L\left(F_{1}^{\prime \prime} \Rightarrow\right)=\left\{\sigma_{1}^{\prime \prime}\right\} \cup \underbrace{L}_{\sigma_{2} \Rightarrow^{+} y \text { in } F_{2}} \mu\left(\mu_{2}(y)\right)$, where $\sigma_{1}^{\prime \prime}$ is in $\mu\left(\mu_{2}\left(\sigma_{2}\right)\right)$.

In other words there is an $F_{2}^{\prime \prime} \underset{f s}{\Delta} F_{2}\left(\mu \mu_{2}\right)$ such that $L\left(F_{2}^{\prime \prime} \Rightarrow\right)=L(F=\Rightarrow)$.

We have shown inclusion in one direction. The inclusion in the other direction follows by a similar argument, hence the result.

To prove the undecidability of the equation

$\mathcal{Z}_{f s}\left(F_{1} \Rightarrow\right)=\mathcal{Z}_{f s}\left(F_{2} \Rightarrow\right)$ it now suffices to prove that it is undecidable whether there exists an $F_{2}^{\prime} \underset{f s}{A} F_{2}$ such that $L\left(F_{2}^{\prime} \Rightarrow\right)=L\left(F_{1} \Rightarrow\right)$. This

we now demonstrate.

\section{Theorem 5.14}

Let $F_{i}=\left(\Sigma_{i}, P_{i}, \sigma_{i}\right) i=1,2$ be two arbitrary $0 L$ forms. Then it is undecidable whether there exists an $F_{2}^{\prime} \underset{f s}{\Delta} F_{2}$ with $L\left(F_{2}^{\prime}, \Rightarrow\right)=L\left(F_{1}, \Rightarrow\right)$.

Proof: We will construct two oL forms which have the desired property iff the PCP has no solution.

Let $\left(x_{1}, \ldots, x_{n}\right),\left(y_{1}, \ldots, y_{n}\right)$ for $n \geq 1$ and $x_{i}, y_{i}$ over $\{a, b\}$ be an arbitrary instance of the PCP. Let $\Sigma_{1}=\Sigma_{2}=\{a, b, S, A, C, D, E, F, *\}$ 
and let the symbols of $\Sigma_{j}-\{S, *\}$ be arbitrarily but uniquely numbered from 1 to 7 , letting $e(c)$ denote this number for each $c$ in $\Sigma_{1}-\{S, *\}$. Let $\sigma_{1}=\sigma_{2}=S$. We first define the productions of $F_{2}$ in two groups:

(1) $\left\{\begin{array}{l}A \rightarrow c B d, \text { for } a 1\} c, d \text { in }\{a, b\}, d \neq c ; \\ B \rightarrow C B ; B+B C, \text { for } c \text { in }\{a, b\}\end{array}\right.$

$S \rightarrow *{ }^{9} x_{i} \operatorname{Emi}\left(y_{i}\right)$, for all $i, 1 \leq i \leq n$;

$S \rightarrow *{ }^{9} C A C$, for a $11 c$ in $\{a, b\}$;

$S \rightarrow *{ }^{9} c B d$, for all $c$, $d$ in $\{a, b\} d \neq c$;

$A \rightarrow C A C ; A \rightarrow C C ; A \rightarrow F C$, for all $c$ in $\{a, b\}$;

$B \rightarrow D$;

$C \rightarrow C C$; for al 1 C in $\{a, b\}$;

$C \rightarrow D ; D \rightarrow D$

$E \rightarrow x_{i} \operatorname{Emi}\left(y_{i}\right)$, for all $i, 1 \leq i \leq n$;

$F \rightarrow F C$, for $a 11 c$ in $\{a, b\}$

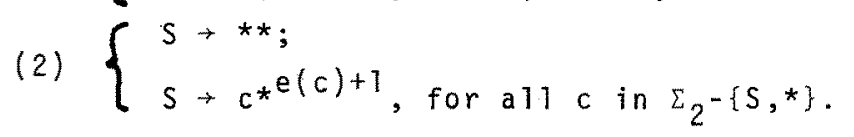

Now we obtain $P_{1}$ by adding the production $E \rightarrow D$ to $P_{2}$.

The reader can readily verify that:

$L\left(F_{1} \Rightarrow\right)=L\left(F_{2} \Rightarrow\right) \cup\left\{*^{9} x_{i_{1}} \ldots x_{i_{t}}\right.$ Dy ${ }_{i_{t}} \ldots y_{i_{1}}: t \geq 1, i_{1}, \ldots, i_{t}$ in $\{1, \ldots, n\}\}$.

Essentially, using the group (1) productions beginning with either $S \rightarrow *{ }^{9} \mathrm{CAC}$ or $S \rightarrow *^{9} \mathrm{CBd}$ leads to words of the type:

${ }^{9} x \operatorname{Dmi}(y)$

where $x \neq y$. Thus the addition of the production $E \rightarrow D$ ensures $L\left(F_{1}, \Rightarrow\right)=L\left(F_{2}, \Rightarrow\right)$ iff the PCP has no solution.

The group (2) productions on the other hand provide detailed structural information. Thus both $F_{1}$ and $F_{2}$ generate only one word of each length from 1 to 9 . S is the only length 1 word, ** the only length 2 word and for each $c$ in $\Sigma_{2}-\{s, *\}$ there is a unique word $c^{*} e(c)+1$ of length $e(c)+2$.

Now assume there is an $F_{2}^{\prime} \underset{\text { fs }}{4} F_{2}(\mu)$ with $L\left(F_{2}^{\prime}, \Rightarrow\right)=L\left(F_{1}, \Rightarrow\right)$. Consider $\mu$. Clearly $S$ is in $\mu(S)$ since $S$ is the only word of length 1 generated by $F_{1}$ and $F_{2}$ and $*$ is in $\mu(*)$ since $* *$ is the only word of length two generated by $F_{1}$ and $F_{2}$. Similarly $c$ is in $\mu(c)$ for each $c$ in $\Sigma_{2}-\{S, *\}$. But this implies $\mu$ is one-to-one and onto, hence $F_{2}^{\prime}=F_{2}$ and therefore $L\left(F_{1}, \Rightarrow\right)=L\left(F_{2} \Rightarrow\right)$. However this implies the $P C P$ has no solution. Turning to the converse argument, assume the PCP has no solution. Then $L\left(F_{1} \Rightarrow\right)=L\left(F_{2}^{\prime} \Rightarrow\right)$ and $F_{2}^{\prime}$ fs $F_{2}$. 
Thus there exists an $F_{2}^{\prime} \underset{f}{f_{s}} F_{2}$ iff the PCP has no solution. However this is undecidable.

\section{Coroltary 5.15}

$$
\begin{aligned}
& \text { Given two } O L \text { forms } F_{1} \text { and } F_{2} \text { it is undecidable whether } \\
& \mathcal{L}_{\mathrm{fs}}\left(F_{1}, \Rightarrow\right)=\mathcal{L}_{\mathrm{fs}}\left(F_{2} \Rightarrow\right) \text {. }
\end{aligned}
$$

We also have:

\section{Corozzary 5.16}

Given two EOL forms $F_{1}$ and $F_{2}$ it is undecidable whether

$\mathcal{L}_{f s}\left(F_{1}, \Rightarrow=\mathcal{L}_{f s}\left(F_{2}, \Rightarrow, \mathcal{L}_{f u}\left(F_{1}, \Rightarrow\right)=\mathcal{L}_{f u}\left(F_{2}, \Rightarrow\right)\right.\right.$ and

$\mathscr{L}_{\mathrm{fwu}}\left(\mathrm{F}_{1}, \Rightarrow\right)=\mathcal{L}_{\mathrm{fwu}}\left(\mathrm{F}_{2}, \Rightarrow\right)$.

Proof: Let $S$ in $F_{1}$ and $F_{2}$ of the proof of Theorem 5.14 be the only nontermina1. Then the result goes through. Finally consider ol-1ike EOL forms in which $S$ is the only nonterminal, that is $S$ is the sentence symbol and it does not appear on the right hand side of a production. Then Theorem 5.14 can be modified for these OL-1ike EOL forms. Hence the result.

We now turn to the positive result, namely given two POL forms one of which has a single letter we can decide if they are s-form equivalent.

$$
\text { We first have: }
$$

\section{Lemma 5.17}

Let $F_{1}=\left(\{a\}, P_{1}, a^{k}\right)$ and $F_{2}=\left(\Sigma_{2}, P_{2}, \sigma_{2}\right)$ be two POL forms.

Then $\mathcal{Z}_{s}\left(F_{1} \Rightarrow \subseteq \mathcal{Z}_{s}\left(F_{2} \Rightarrow\right)\right.$ iff there is some $b$ in $\Sigma_{2}$ such that $\sigma_{2}=b^{k}$ and $b \rightarrow b^{r}$ is in $P_{2}$ for each production $a \rightarrow a^{r}$ in $P_{1}$.

Proof: if: obvious.

only if: Consider some production $a \rightarrow a^{r}$ in $P_{1}$ and $F_{1}=\left(\{a\},\left\{a \rightarrow a r_{, a}{ }^{k}\right)\right.$. Since $\mathcal{L}_{s}\left(F_{1}, \Rightarrow \subseteq \mathcal{L}_{s}\left(F_{2}, \Rightarrow\right)\right.$ there is an $F_{2}^{i} F_{2}$ with $L\left(F_{2}^{\prime}, \Rightarrow\right)=L\left(F_{j}^{\prime}, \Rightarrow\right)$.

Case 1: $r=1$. Then $L\left(F_{1}^{\prime}, \Rightarrow\right)=\left\{a^{k}\right\}$ hence $\sigma_{2}=b^{k}$ for some $b$ in $\Sigma_{2}$ and $b \rightarrow b$ is in $P_{2}$. 
Case 2: $r>1$. Then $L\left(F_{j}^{\prime} \Rightarrow\right)=\left\{a^{k r^{i}}: i \geq 1\right\}$, hence $\sigma_{2}=b^{k}$ for some $b$ in $\Sigma_{2}$ and since $a^{k} \Rightarrow a^{k r}$ in $F_{1}^{\prime}$ where $a^{k r}$ is the second smallest word in $L\left(F_{1}, \Rightarrow\right.$ we must have $b^{k} \Rightarrow b^{k r}$ in $F_{2}$, that is $b \rightarrow b^{r}$ is in $F_{2}$.

Thus the lemma is proved.

In the following we can now assume that $a$ is in $\Sigma_{2}$ and $\sigma_{2}=a^{k}$, without any loss of generality. We say that an $0 L$ form $F=(\Sigma, P, \sigma)$ is production minimal if there is no $F^{\prime}=\left(\Sigma, P^{\prime}, \sigma\right), P^{\prime} \nsubseteq P$ such that $F$ and $F^{\prime}$ are strong $s-$ form equivalent. By the techniques of section II.3.4 a production minimal OL form $F^{\prime}$ can always be obtained from an $O L$ form $F$ such that $F$ and $F^{\prime}$ are strong $s$-form equivalent.

\section{Theorem 5.18}

Let $F_{1}=\left(\{a\}, P_{1}, a^{k}\right)$ and $F_{2}=\left(\Sigma_{2}, P_{2}, a^{k}\right)$ be POL forms where $F_{2}$ is production minimal. Then $\mathcal{Z}_{s}\left(F_{1} \Rightarrow\right)=\mathcal{Z}_{s}\left(F_{2} \Rightarrow\right)$ implies $P_{1}=P_{2}$.

Proof: By the previous lemma we know that $P_{1} \subseteq P_{2}$, therefore it suffices to prove that $\mathrm{P}_{2}-\mathrm{P}_{1}=\emptyset$.

Assume $P_{2}-P_{1} \neq \varnothing$ and $a \rightarrow x$ is in $P_{2}-P_{1},|x|=\ell$. Clearly if $a \rightarrow a^{l}$ is in $P_{1}$ this would imply that $P_{2}$ is not production minimal. Therefore assume $a \rightarrow a^{l}$ is not in $P_{1}$.

(1) $x=a^{\ell}$. An immediate contradiction since $\left\{a^{k \ell^{i}}: i \geq 1\right\}$ is not in $\mathcal{L}_{s}\left(F_{1}, \Rightarrow\right)$.

(2) $x=x_{1} b x_{2}$ for some $b$ in $\Sigma_{2}-\{a\}$. Let $\bar{x}$ denote $x$ with a replaced everywhere with $\bar{a}$ and consider the $\mathrm{OL}$ form $F_{2}^{\prime}=\left(\Sigma_{2},\left(\overline{P_{2}-P_{1}}\right) \cup\{a \rightarrow \bar{x}, \bar{a} \rightarrow \bar{x}\}, a^{k}\right)$, where $\overline{P_{2}-P_{1}}$ is $P_{2}-P_{1}$ with a replaced everywhere with $\bar{a}$. Then $L=L\left(F_{2}^{\prime} \Rightarrow\right)=\left\{a^{k}, \bar{x}^{k}\right\}$ u $\left\{y: \bar{x}^{k} \Rightarrow y\right.$ and $\left.|y| \geq k \ell\right\}$. Since $\mathcal{L}_{s}\left(F_{1} \Rightarrow\right)=\mathcal{L}_{s}\left(F_{2}, \Rightarrow\right)$ there is an $F_{1} \Delta_{s} F_{1}$ with $L\left(F_{1}, \Rightarrow\right)=L\left(F_{2}^{\prime}, \Rightarrow\right)$. Since $a^{k}$ is the smallest word in $L, a^{k}$ is the axiom of $F_{j}^{\prime}$ and there is some word $y$ in $L$ such that $|y|=k \ell$ and $a^{k} \Rightarrow y$ in $F_{j}^{\prime}$. Now $a \rightarrow y_{i}$ is in $P_{j}^{i}, 1 \leq i \leq k$, and since $a \rightarrow a^{l}$ is not in $P_{1}$, we have $\left|y_{i}\right| \neq l, 1 \leq i \leq k$. Therefore there is at least one $y_{i}$ with $\left|y_{i}\right|<\ell$ in which case we have $a^{k} \Rightarrow y_{i}^{k}$ with $k \leq\left|y_{j}^{k}\right|<k \ell$. Hence either there are two distinct words of length $k$ in $L_{(}\left(F_{j}, \Rightarrow\right.$ or there is a word of length properly between $k$ and $k \ell$ in $L\left(F_{j}^{\prime} \Rightarrow\right)$. 
In both cases $L\left(F_{1}^{\prime} \Rightarrow\right) \neq L\left(F_{2}^{\prime}, \Rightarrow\right)$. Thus $L\left(F_{2}^{\prime}, \Rightarrow\right)$ is not in $\mathcal{L}_{\mathrm{s}}\left(\mathrm{F}_{1}, \Rightarrow\right)$.

In both cases we have arrived at a contradiction namely,

$\mathcal{L}_{\mathrm{s}}\left(F_{1}, \Rightarrow\right) \neq \mathcal{L}_{\mathrm{s}}\left(F_{2}, \Rightarrow\right)$, therefore $P_{1}=P_{2}$.

We now have a decision procedure for form equivalence when one of the POL forms is a one-letter form.

\section{Theorem 5.19}

Let $F_{1}=\left(\{a\}, P_{1}, a^{k}\right)$ and $F_{2}=\left(\Sigma_{2}, P_{2}, \sigma_{2}\right)$ be POL forms. Then it is decidable whether $\mathcal{L}_{s}\left(F_{1}, \Rightarrow\right)=\mathcal{L}_{s}\left(F_{2}, \Rightarrow\right)$.

Proof: If $\sigma_{2} \neq b^{k}$ for some $b$ in $\Sigma_{2}$ then we have inequivalence by Lemma 5.17. Therefore assume $\sigma_{2}=b^{k}$. Using the techniques of Section II.3.4 we can effectively determine $F_{2}^{\prime}$ a sub-OL form of $F_{2}$ which is production minimal and which is strong s-form equivalent to $F_{2}$. Now applying Theorem $5.18 P_{1}=P_{2}^{i}$ iff $F_{1}$ and $F_{2}$ are form equivalent. Clearly this is decidable. 


\section{III.6 Generative Capacity of EOL Forms}

We have four aims in this section. First, in Section 6.1, we briefly examine closure and non-closure results. Second, in Section 6.2, we demonstrate that every sub-regular grammatical family is also an EOL form family and third, in Section 6.3, we consider regular-completeness. In this third part the weaker notions of regular-sufficiency and regular-boundedness are introduced and some first results given. Finally in section 6.4 we consider the notion of a generator. We prove that there is no generator for $\mathcal{L}(E O L)$ and no proper generators for $\mathcal{Z}($ LIN ) and $\mathcal{L}(\mathrm{CF})$.

\section{III.6.1 Closure and Non-closure Properties}

As is the case for grammar forms under s-interpretation, we mainly have non-closure properties. However in the case of grammar forms we always have closure under intersection with a regular set. This is not true for EOL forms in general.

That we may obtain AFL's is seen by considering the following EOL form:

$$
S \rightarrow a S ; S \rightarrow a ; a \rightarrow a
$$

which has been shown to be a regular-complete in III.3.4.

Conversely, we may also contain anti-AFL's, for example, consider $F$ defined by:

$$
\begin{aligned}
& S \rightarrow a ; S \rightarrow c C ; S \rightarrow A A A A ; A \rightarrow A A ; A \rightarrow b ; \\
& a \rightarrow a ; b \rightarrow b ; c \rightarrow c .
\end{aligned}
$$

Consider the languages $L_{1}=\left\{a_{1}, a_{2} a_{2}\right\}$ and $L_{2}=\left\{a_{1} a_{1}, a_{2}\right\}$. Both are in $\mathcal{L}_{s}(F, \Rightarrow)$, for example $F_{1}$ defined by:

$$
s_{1} \rightarrow a_{1} ; s_{1} \rightarrow a_{2} a_{2} ; a_{1} \rightarrow a_{1} ; a_{2} \rightarrow a_{2}
$$
is an interpretation of $F$ and $L\left(F_{1}, \Rightarrow\right)=L_{1}$. However $L_{1} \cup L_{2}=\left\{a_{1}, a_{2}, a_{1} a_{1}, a_{2} a_{2}\right\}$ is not in $\mathcal{L}_{s}(F, \Rightarrow)$ as can be seen readily. Since 1-symbol words can only be obtained from interpretations of $S \rightarrow a$ and 2-symbol words from $S \rightarrow c c$, we must have a and $c$ interpreted as $a_{1}$ and $a_{2}$ if $L_{1} \cup L_{2}$ is in $\mathcal{L}_{s}(F, \Rightarrow)$. This contradicts the definition of intepretation, hence $\mathcal{L}_{S}(F, \Rightarrow)$ is not closed under union. Now observe the following facts concerning $F$.

(i) $L(F, \Rightarrow)$ contains no word of length 3 .

(ii) If $L$ is in $\mathcal{L}_{S}(F, \Rightarrow$ then the length set of $L$ is ultimately periodic.

Fact (i) follows directly from the productions of $F$, therefore consider (ii). Let $L$ be in $\mathcal{L}_{S}(F, \Rightarrow)$, that is $L=L\left(F^{\prime}, \Rightarrow\right)$ for some $F^{\prime} \oiint_{S} F$. 
Consider the EOL grammar $G$ obtained from $F^{\prime}$ by replacing all terminals appearing in the productions of $F^{\prime}$ by one and the same terminal $a$. Clearly the length set of $L(G, \Rightarrow)$ equals the length set of $L$. Now $L(G, \Rightarrow)$ is context-free since the only production for a is a $\rightarrow$ a; that is it is stable with respect to terminals. Since $G$ is unary, $L(G, \Rightarrow)$ is regular and hence the length set of $L(G, \Rightarrow)$ is an ultimately periodic set by well known results.

Consider once more the language $L_{1}=\left\{a_{1}, a_{2} a_{2}\right\}$ in $\mathcal{L}_{s}(F, \Rightarrow)$. since $L_{1} L_{1}$ and $L_{1}^{*}$ contain words of length $3, \mathcal{L}_{S}(F \Rightarrow)$ is not closed under product or star.

$$
L_{3}=\left\{a_{1}, a_{2}, a_{3} a_{3}, a_{4} a_{4}\right\} \text { is readily seen to be in } \mathcal{Z}_{5}(F, \Rightarrow) \text {. }
$$

Define a $\lambda$-free homomorphism $h$ by:

$$
h\left(a_{1}\right)=a_{1} ; h\left(a_{2}\right)=a_{2} ; h\left(a_{3}\right)=a_{1} ; h\left(a_{4}\right)=a_{2},
$$

then we have $h\left(L_{3}\right)=L_{1} \cup L_{2}$ is not in $\mathcal{L}_{s}(\mathrm{~F}, \Rightarrow)$.

Similarly $L_{4}=\{$ bbdd $\}$ is easily seen to be in $\mathcal{L}_{S}$ (F, $\Rightarrow$ ) and defining

the homomorphism $h$ by:

$$
h(a)=b b ; h(c)=d
$$

we have

$$
h^{-1}\left(L_{4}\right)=\{a c c\} \text { is not in } \mathcal{L}_{s}(F, \Rightarrow) \text {. }
$$

Thus $\mathcal{L}_{\mathrm{S}}(\overrightarrow{\mathrm{F}} \Rightarrow)$ is not closed under homomorphism or inverse homomorphism.

Finally, consider $F^{\prime} \underset{s}{\Delta} F$ having the productions

$S \rightarrow A A A A ; A \rightarrow A A ; A \rightarrow b ; b \rightarrow c ; c \rightarrow c$.

Since $L\left(F^{\prime}, \Rightarrow\right) b^{*}=\left\{b^{2^{n}}: n \geq 2\right\}$ and $\left\{2^{n}: n \geq 2\right\}$ is not uTtimately periodic, $\mathcal{Z}_{s}(F, \Rightarrow$ is not closed under intersection with regular sets.

Hence we have demonstrated that $\mathcal{L}_{s}(F, \Rightarrow)$ is an anti-AFL.

However if we turn to synchro-EOL forms we always have closure under intersection with regular sets. This can be proved in the standard way, cf. Section II.4.2.

Similarly if we only deal with unary EOL forms we have closure under union. We summarize these results in

\section{Theorem 6.1}

(i) There are EOL forms $F$, such that $\mathcal{L}_{S}(F, \Rightarrow$ is an $A F L$ or an anti-AFL.

(ii) For $F$ an arbitrary synchro-EOL form, $\mathcal{W}_{S}(F, \Rightarrow$ is closed under intersection with regular sets.

(iii) For $F$ an arbitrary unary EOL form, $\mathscr{L}_{s}(F, \Rightarrow$ ) is closed under union. 
III.6.2 Sub-regular Grammatical Families are EOL Form Families

To demonstrate that sub-regular grammatical families are indeed EOL form families, we first need to prove a normal form theorem for sub-regular grammar forms. In fact we will show that the grammar form

$$
S \rightarrow a S ; S \rightarrow a
$$

is regular-vomplete.

Recall the following definition:

Let $G=(V, \Sigma, P, S)$ be a grammar form. We say $G$ has an $(A, \alpha, B, B)=$ partition, for some $A, B$ in $V-\Sigma$ and $\alpha \beta$ in $V *$, if the following conditions hold:

(i) $A \rightarrow \alpha B B$ is in $P$,

(ii) $V=\bar{V}$ i $\bar{V}$, where $\overline{\bar{V}} \subseteq V-\Sigma, A$ and $S$ are in $\bar{V}, B$ is in $\bar{V}$ and $\alpha \beta$ is in $\bar{V}^{*}$,

(iii) $P=\bar{P} \dot{U}\{A \rightarrow a B B\} \dot{U} \overline{\bar{P}}$, where $\bar{P} \underline{c}(\bar{V}-\Sigma) \times \bar{V}$ * and

$\overline{\overline{\mathrm{P}}} \subseteq \overline{\overline{\mathrm{V}}} \times(\overline{\overline{\mathrm{V}}} \cup \Sigma)^{*}$.

Lemma II.2.20 demonstrates that for each grammar form $F$ with a production $A \rightarrow a B B$ a form equivalent grammar form $G$ can be obtained which has a production $A \rightarrow \alpha B^{\prime} B$ in $p l a c e$ of $A \rightarrow \alpha B B$, where $B^{\prime}$ is a new nonterminal and moreover $G$ has an $\left(A, \alpha, B^{\prime}, B\right)$-partition.

The importance of such a partition is that the productions used in the subgrammar $G_{B^{\prime}}$ of $G$ can only be used in a sentential derivation of $G$ if the production $A \rightarrow \alpha B^{\prime} B$ is first used.

We make much use of this result in the following proof. We first need to recall the definition of the natural pre-order on the nonterminals of a grammar form.

Let $G=(V, \Sigma, P, S)$ be a grammar form. We say $A \leq B$ if $A \Rightarrow^{*} \alpha B \beta$, for some $\alpha$ and $B$, where $A$ and $B$ are nonterminals. We define a relation $\sim$ on $V-\sum$ by: $A \sim B$ iff $A \leq B$ and $B \leq A$. Clearly $\sim$ is an equivalence relation. Letting $[A]_{\sim}$ denote the equivalence class of $A$ with respect to $\sim$, we extend $\leq$ to equivalence classes by:

$[A]_{\sim} \leq[B]_{\sim}$ iff $A^{\prime} \leq B^{\prime}$ for some $A^{\prime}$ in $[A]_{\sim}$ and $B^{\prime}$ in $[B]_{\sim}$.

Clearly $\leq$ is a partial order of equivalence classes.

We can now prove the needed vompleteness result.

\section{Theorem 6.2}

Let $F$ be a grammar form defined by the productions $S \rightarrow a$ and $S \rightarrow a S$.

Then for every grammar form $G=(V, \Sigma, P, Z)$ with $\mathscr{L}_{s}(G, \Rightarrow) \subseteq \mathcal{L}(R E G)$ there exists $F^{\prime} \vec{S} F$ form equivalent to $G$. 
Proof: We may assume, by the results of Section II.4.1 that $G$ is reduced, $\lambda$-free, chain-free and non-left recursive. We prove the result by induction on the number of equivalence classes of $V-\Sigma$ under the relation 2 .

Basis: $[Z]_{\varkappa}=V-\Sigma$.

By the assumptions $G$ is non-left-recursive, hence each production in $P$ is of the type:

$A \rightarrow a \alpha$, $a$ in $\Sigma$ and $\alpha$ in $V *$.

We now show that $\alpha$ is in $\Sigma * u \Sigma^{*}(V-\Sigma)$. Assuming otherwise then either a contains two nonterminals or $\alpha$ is in $\Sigma^{*}(V-\Sigma) \Sigma^{+}$. In the former case, $G$ is expansive, hence $\mathcal{L}_{S}(G, \Rightarrow)$ is not sub-regular; a contradiction. In the latter case we have:

$$
A \rightarrow a \alpha_{1} B \alpha_{2} b \text { in } P \text {, for some } B \text { in } V-\Sigma \text { and } a, b \text { in } \Sigma \text {, }
$$
nence $\mathcal{L}_{\mathrm{S}}(\mathrm{G}, \Rightarrow)$ contains linear languages, a contradiction.

Thus we can assume the productions in $P$ are of the type:

$A \rightarrow a \alpha$, a in $\Sigma, \alpha$ in $\Sigma^{*} u \Sigma^{*}(V-\Sigma)$.

Construct a new grammar form $H$ from $G$ as follows:

(i) For each production $A \rightarrow a_{1} \ldots a_{m}$ in $P, m \geq 2, a_{i}$ in $\Sigma$, $1 \leq i \leq m$, take the productions:

$$
A \rightarrow a_{1}\left[a_{2} \ldots a_{m}\right] ;\left[a_{2} \cdots a_{m}\right] \rightarrow a_{2}\left[a_{3} \ldots a_{m}\right] ;\left[a_{m}\right] \rightarrow a_{m}
$$
into $H$, where the $\left[a_{i} \ldots a_{m}\right], 2 \leq i \leq m$ are new nonterminais.

(ii) For each production $A \rightarrow a_{1} \ldots a_{m} B$ in $P, m \geq 2, a_{i}$ in $\Sigma$, $1 \leq i \leq m$ and $B$ in $V-\Sigma$, take the productions:

$A \rightarrow a_{1}\left[a_{2} \ldots a_{m} B\right] ;\left[a_{2} \cdots a_{m} B\right] \rightarrow a_{2}\left[a_{3} \cdots a_{m} B\right] ; \ldots ;\left[a_{m} B\right] \rightarrow a_{m} B$ into $H$, where the $\left[a_{i} \ldots a_{m} B\right], 2 \leq i \leq m$ are new nonterminals.

(iii) For each production $A \rightarrow a$ or $A \rightarrow a B$ in $P$, a in $\Sigma$, $B$ in $V-\Sigma$ take it unchanged into $H$.

Clearly $H \underset{S}{\triangle} F$ and by simulation $\mathcal{L}_{\mathrm{s}}(G, \Rightarrow) \subseteq \mathcal{L}_{\mathrm{S}}\left(H_{s} \Rightarrow\right)$.

That $\mathcal{X}_{s}(H, \Rightarrow) \subseteq \mathcal{Z}_{s}(G, \Rightarrow)$ follows easily from the Back-substitution Lemma, Lemma II.2.12. Hence the Basis is complete.

Inductive Hypothesis: Assume the Theorem is true for al1 $G$ with at most $k \geq 1$ equivalence classes.

Inductive Step: Assume $G$ has $k+1$ equivalence classes.

Now $G$ has at least 2 equivalence classes and for all $\mathrm{A}$ in $V-\Sigma,[Z]_{\imath} \leq[A]_{\Omega}$.

We use similar arguments to those used above.

For all A in $[Z]_{\varkappa}$, for each production: 
$A \rightarrow \alpha$ in $P$,

$a$ is in $\left(V-[Z]_{n}\right)^{+} u\left(V-[Z]_{n}\right)^{+}[Z]_{\Omega}$.

For otherwise either a contains two [Z] $]^{-s y m b o l s}$ or $\alpha$ is in $\left(v-[z]_{\imath}\right)^{+}[z]_{\Omega}\left(v-[z]_{\Omega}\right)^{+}$.

In the first case $G$ is expansive giving an immediate contradiction while in the second case $G$ is self-embedding again giving a contradiction.

Consider a production $A \rightarrow \alpha$ with $A$ in $[Z]_{\Omega}$. First of all by Lemma II.2.20 we may assume without loss of generality that if $\alpha$ contains a nonterminal $B$ in $V-[Z]_{\Omega}$, that is $\alpha=\alpha_{1} B \alpha_{2}$, then $G$ has an $\left(A, \alpha_{1}, B, \alpha_{2}\right)$-partition. Furthermore by the Replacement Lemma (Lemma II.2.19) and the inductive assumption we may assume that the subgrammar $G_{B}$ of $G$ is an interpretation of $F$.

Thus we only have to modify the productions for nonterminals in $[Z]_{\Omega}$. We do this in two steps.

First, let $A \rightarrow B \alpha$ be in $P$ with $A$ in $[Z]_{2}$, and $B$ in $(V-\Sigma)-[Z]_{\sim}$. By the Back-Substitution Lemma (Lemma II.2.12) we may replace $A \rightarrow B \alpha$ in $P$ by the productions:

$$
A \rightarrow B \alpha \text {, for all } B \rightarrow B \text { in } P \text {, }
$$

giving a form equivalent grammar form.

Carry out this substitution for all such productions $A \rightarrow B \alpha$ in $P$ with $A$ in $[Z]_{n}$ and $B$ in $(V-\Sigma)-[Z]_{n}$ and let the resulting grammar form be $G_{1}=\left(V, \Sigma, P_{1}, Z\right)$.

As a result of this transformation it is now true that for all productions $A \rightarrow \alpha_{1}$ in $P_{1}$ with $A$ in $[Z]_{\vartheta}$,

$\alpha_{1}=a \alpha$, a in $\Sigma$ and $\alpha$ in $\left(V-[Z]_{\Omega}\right) * u\left(V-[Z]_{\Omega}\right) *[Z]_{\Omega}$.

We construct a new grammar $H$ from $G_{1}$ as follows:

(1) For a 11 productions $A \rightarrow a X_{T} \ldots X_{m}$ in $P$ with $A$ in $[Z]_{n}$,

$m \geq 0$, take the following productions into $H$ :

(i) $A \rightarrow a$ if $m=0$

(ii) $A \rightarrow a X_{1}$ if $m=1$ and $X_{1}$ is in $V-\Sigma$, and

(iii) $A \rightarrow a\left[x_{1} \ldots x_{m}\right]$ if either $m \geq 2$ or $\left(m=1\right.$ and $x_{1}$ is in $\Sigma$ ).

(2) For a $11 W_{1} \ldots W_{r}, 1<r \leq n$, where $n=\max (\{|\alpha|: A \rightarrow$ a a in $P\})$, $W_{j}$ is in $V-[Z]_{\imath}, 1 \leq i<r$ and $W_{r}$ is in $V$, take into $H$ the productions:

(i) $\left[W_{1} \ldots W_{r}\right] \rightarrow a\left[Y_{1} W_{2} \ldots W_{r}\right]$, if $W_{1} \rightarrow a Y_{1}$ is in $P$,

(ii) $\left[W_{1} \ldots w_{r}\right] \rightarrow a\left[W_{2} \ldots w_{r}\right]$, if $W_{1} \rightarrow a$ is in $p$ and $r \geq 2$, and

(iii) $\left[W_{1} W_{2}\right] \rightarrow a W_{2}$ if $W_{1} \rightarrow a$ is in $P$

(3) Take all other productions in $\mathrm{P}$ unchanged into $\mathrm{H}$.

clearly $H \underset{s}{\leftrightarrow}$ F. That $\mathscr{L}_{s}\left(G_{1}, \Rightarrow\right)=\mathcal{L}_{s}(H, \Rightarrow)$ follows by observing 
that the tuples in $H$ keep track of left sentential derivations. The reader is invited to provide a rigorous proof.

Since $\mathcal{L}_{s}\left(G_{1}, \Rightarrow\right)=\mathcal{L}_{s}(G, \Rightarrow)$ we have completed the induction step and also the theorem.

We have an immediate corollary.

\section{Coroltary 6.3}

$$
\mathrm{H}_{1}: \mathrm{S} \rightarrow \mathrm{a} ; \mathrm{S} \rightarrow \mathrm{aS} \text { and } \mathrm{H}_{2}: \mathrm{S} \rightarrow \mathrm{a} ; \mathrm{S} \rightarrow \mathrm{Sa}
$$

are regular-vomplete grammar forms.

We are now in a position to prove the main result of this section, namely:

\section{Theorem 6.4}

Every grammatical family $\mathcal{L} \subseteq \mathcal{L}(R E G)$ is generated by a synchro-EOL form.

Proof: By Theorem 6.2 we may assume $\mathcal{L}=\mathcal{L}_{s}(G, \Rightarrow)$, where $G=(V, \Sigma, P, s)$ is a right-linear grammar form. Now introduce for each letter $a$ in $\Sigma$ a new nonterminal [a] and consider the synchro-EOL form $F=\left(V \cup\{[a]:\right.$ a in $\Sigma\}, \Sigma, P^{\prime} \cup\{[a] \rightarrow[a],[a] \rightarrow a$; a in $\left.\Sigma\}, S\right)$, where $P^{\prime}$ is obtained from $P$ by replacing every appearance of a terminal symbol a occurring on the right side of a production with the coresponding nonterminal [a].

We claim $\mathcal{L}_{s}(G, \Rightarrow)=\mathcal{L}_{s}(F, \Rightarrow)$. The inclusion $\mathcal{L}_{s}(G, \Rightarrow) \subseteq \mathcal{Z}_{s}(F, \Rightarrow)$ is obvious, therefore consider the reverse inclusion. Let $F^{\prime}$ be an interpretation of $F$. We define first the "corresponding" interpretation $G^{\prime}$ of $G$. Determine for each interpretation $A$ of [a] the set of terminals $b$ such that $A \rightarrow b$ is a production in $F^{\prime}$. Now replace every occurrence of $A$ on the right side of a production in $F^{\prime}$ in al1 possible ways by such terminals b and finally omitting all productions with $A$ or a terminal letter or $N$ on the left hand side.

That $L\left(F^{\prime} \Rightarrow\right) \subseteq L\left(G^{\prime} \Rightarrow\right)$ is clear. In general the converse is not true, however. $L\left(F^{\prime}, \Rightarrow\right)=M\left(L\left(G^{\prime}, \Rightarrow\right)\right)$ for some gsm mapping $M$ as in Theorem 3.29. It is easy to see that such a gsm can be realized as a composition of dfl-substitutions and intersections with regular languages. Since $\mathcal{L}_{s}(G, \Rightarrow)$ is closed under both these operations we conclude that there is a $G^{\prime \prime} \underset{S}{\triangle}$ such that $L\left(G^{\prime \prime}, \Rightarrow\right)=M\left(L\left(G^{\prime}, \Rightarrow\right)\right)$. 
Theorem 6.4 can be generalized to every sub-linear grammatical family by similar techniques. Whether the converse of Theorem 6.4 holds is still unanswered. For arbitrary EOL forms it is clearly not true since "terminal forcing" may be present.

\section{III.6.3 Regular-completeness, -boundedness and-sufficiency}

Given an EOL form $F$ we say it is regular-bounded if $\mathcal{L}_{S}\left(F, \Rightarrow \subseteq \mathcal{L}(R E G)\right.$, while it is regular-sufficient if $\mathcal{L}_{S}(F, \Rightarrow \geq \mathcal{X}(R E G)$. Hence $F$ is regular-complete if it is both regular-bounded and regularsufficient. From section 3.4 we know that

and

$$
\mathrm{F}_{1}: \mathrm{S} \rightarrow \mathrm{aS} ; \mathrm{S} \rightarrow \mathrm{a} ; \mathrm{a} \rightarrow \mathrm{a}
$$

$$
\mathrm{F}_{2}: \mathrm{S} \rightarrow \mathrm{Sa} ; \mathrm{S} \rightarrow \mathrm{a} ; \mathrm{a} \rightarrow \mathrm{a}
$$

are regular-complete hence both are regular-bounded and regularsufficient. Immediately we also have that:

$$
\mathrm{F}_{3}: \mathrm{S} \rightarrow \mathrm{aS} ; \mathrm{S} \rightarrow \mathrm{b} ; \mathrm{a} \rightarrow \mathrm{a} ; \mathrm{b} \rightarrow \mathrm{b}
$$

is regular-bounded since $\mathrm{F}_{3} \underset{\mathrm{s}}{\overrightarrow{F_{1}}} \mathrm{~F}_{1}$ and $\mathrm{F}_{1}$ is regular-bounded, however $\mathrm{F}_{3}$ is not regular-sufficient since the language \{aa\} cannot be obtained from any interpretation of $\mathrm{F}_{3}$. Conversely

$$
\mathrm{F}_{4}: \mathrm{S} \rightarrow \mathrm{SS} ; \mathrm{S} \rightarrow \mathrm{a} ; \mathrm{a} \rightarrow \mathrm{a}
$$

is regular-sufficient, in fact context-free-sufficient, but $F_{4}$ is not regular-bounded.

The notions of boundedness and sufficiency while of interest in their own right also serve to reduce the problem of the decidability of completeness to two hopefully simpler decidability problems. However at the time of writing little is known about either of these problems. Therefore we only investigate them for a very restricted class of EOL forms in the remainder of this section.

We say a two-symbol form $F=(\{S, a\},\{a\}, P, S)$ is super short if $P \subseteq\{S, a\} \times\{S, a, a S, S a, S S\}$.

Let us first consider regular-sufficiency for a super short form $F$.

Clearly we must have $S \rightarrow$ a in $F$, otherwise $L(F, \Rightarrow)=\emptyset$.

Therefore we assume its presence in the following.

Case 1: $P \cap\{S \rightarrow a S ; S \rightarrow S a ; S \rightarrow S S\}=\emptyset$.

$P$ contains at most the productions $S \rightarrow$ a and $S \rightarrow S$ for $S$, therefore consider the derivation of the word a a in F. Since a must be derived from a, that is 


$$
S \rightarrow a \Rightarrow+a a \text { in } F
$$

the language $\{a a\}$ is unobtainable from any interpretation of $F$. Thus $F$ is not regular-sufficient.

Hence for $F$ to be regular-sufficient one of the productions $S \rightarrow$ aS, $S \rightarrow S a$ or $S \rightarrow S S$ must be present.

Case 2: $P \cap\{S \rightarrow$ aS; $S \rightarrow S a ; S \rightarrow S S\} \neq \emptyset$.

Case 2.1: $\quad P \cap\{S \rightarrow$ aS; $S \rightarrow S a ; S \rightarrow S S\}=\{S \rightarrow$ aS $\}$

We now proceed by examining the possible productions for a.

(a) $a \rightarrow a$ is in $P$.

$F$ is regular-sufficient since $F_{1}$ above is regular-sufficient anf $F_{1}>_{S} F$.

(b) $a \rightarrow S$ and $S \rightarrow S$ are in $P$.

Then $F$ is regular-sufficient. This can be seen by observing that any right linear grammar can be simulated by some interpretation of $F$. Thus letting $G=(V, \Sigma, Q, Z)$ be a right linear grammar construct $F^{\prime}=\left(V^{\prime}, \Sigma, P^{\prime}, Z\right)$ as follows:

$V^{\prime}=V \cup\{\bar{a}: a$ in $\Sigma\}$ and $P^{\prime}$ contains the productions

(i) $A \rightarrow a B$ if $A \rightarrow a B$ is in $Q$,

(ii) $A \rightarrow \bar{a}$ if $A \rightarrow a$ is in $Q$, and

(iij) $a \rightarrow \bar{a}, \bar{a} \rightarrow \bar{a}, \bar{a} \rightarrow a$ if $a$ is in $\Sigma$.

Now if $Z \Rightarrow^{+} x$ in $G$, for $x$ in $\Sigma^{+}$, it should be clear that $Z \Rightarrow^{+} x$ in $F^{\prime}$ and conversely. Since $F^{\prime} \vec{s} F$ by construction, $F$ is regular-sufficient.

(c) $P \cap\{a \rightarrow a ; s \rightarrow S\}=\emptyset$.

Consider the word aa.

If $a \rightarrow S S$ is not in $P$ then aa is not in $L(F, \Rightarrow)$, hence $F$ is not regular-sufficient. On the other hand if $a \rightarrow S S$ is in $P$ then every derivation for aa is of the type:

$S \Rightarrow^{t} a \Rightarrow^{t}$ a a

in which case $\{a a\}$ is not in $\mathcal{L}_{s}(F, \Rightarrow)$ and again $F$ is not regularsufficient.

(d) $P \cap\{a \rightarrow a ; a \rightarrow S\}=\emptyset$.

Again by considering the word a it can be shown that $F$ is not regular-sufficient.

Thus we have shown the following:

\section{Lemma 6.5}

Let $F=(\{S, a\},\{a\}, P, S)$ be a supershort form such that $\{S \rightarrow a ; S \rightarrow a S ; S \rightarrow S a ; S \rightarrow S S\} \cap P=\{S \rightarrow a ; S \rightarrow a S\}$. 
Then $F$ is regular-sufficient iff $\{a \rightarrow a\} \subseteq P$ or $\{a \rightarrow S ; S \rightarrow S\} \subseteq P$.

Proof: if: (a) and (b) above give this result.

only if: (c) and (d) give this.

Clearly weakening the initial condition on $P$ to

$\{S \rightarrow a ; S \rightarrow a S ; S \rightarrow S a ; S \rightarrow S S\} \cap P=\{S \rightarrow a ; S \rightarrow a S ; S \rightarrow S a\}$

does not affect the validity of this Lemma. Hence we are only left with the following case.

Case 2.2: $P \cap\{S \rightarrow$ aS; $S \rightarrow S a ; S \rightarrow S S\}=\{S \rightarrow S S\}$.

(a) $a \rightarrow a$ is in $P$.

As in Case $2.1(a)$ this is regular-sufficient since $F_{4} \underset{s}{\circlearrowleft} F$.

(b) $S \rightarrow S$ is in $P$.

Proceed as in Case $2.1(b)$ to construct an $F^{\prime} \underset{s}{\Delta} F$ from a given right linear grammar $G=(V, \Sigma, Q, Z)$. However $P^{\prime}$ contains the productions:

(i) $A \rightarrow \bar{B} B$ if $A \rightarrow a B$ is in $Q$,

(ii) $A \rightarrow a$ if $A \rightarrow$ a is in $Q$,

(iii) $\bar{a} \rightarrow \bar{a}, \bar{a} \rightarrow a$ for all a in $\Sigma$.

Now $L\left(F^{\prime} \Rightarrow\right)=L(G, \Rightarrow)$ and $F^{\prime}{\underset{5}{5}}_{F}$ by construction. Hence $F$ is regular-sufficient.

(c) $\quad P \cap\{a \rightarrow a ; s \rightarrow s\}=\emptyset$.

Consider the word aa and possible derivations for it in $F$.

If the production $S \rightarrow$ a is first to be applied then we have:

$S \Rightarrow a \Rightarrow^{+}$a a a

otherwise if $S \rightarrow S S$ is the first production to be applied then we have:

$S \Rightarrow S S \Rightarrow^{+}$aaa.

This is a valid derivation only when the second step is:

$S \Rightarrow S S \Rightarrow a a \Rightarrow^{+}$aa.

In both cases because of "terminal forcing" the language \{aa\}

is not in $\mathcal{L}_{\mathrm{s}}(\mathrm{F}, \Rightarrow)$.

Case 2.2 can be summarized as follows:

\section{Lemma 6.6}

Let $F=(\{S, a\},\{a\}, P, S)$ be a supershort form such that $\{S \rightarrow a ; S \rightarrow a S ; S \rightarrow S a ; S \rightarrow S S\} \cap P=\{S \rightarrow a ; S \rightarrow S S\}$. Then $F$ is regular-sufficient iff $\{a \rightarrow a ; S \rightarrow S\} \cap P \neq \emptyset$. 
Thus for a supershort form $F$ we can decide whether or not $F$ is regular-sufficient by combining Lemmas 6.5 and 6.6 .

Let us now turn to the study of regular-boundedness of supershort forms.

Let $F=(\{S, a\},\{a\}, P, S)$ be a supershort form. Again we assume throughout that $S \rightarrow$ a is in $P$, since otherwise $L(F, \Rightarrow)=\emptyset$ and $F$ is trivially regular-bounded.

Case 1: $a \rightarrow$ SS is in P.

Consider the form $F^{\prime}$ os $F$ with the two productions $S \rightarrow$ a and $a \rightarrow S S . L\left(F^{\prime} \Rightarrow\right)$ is non-regular. Hence $F$ is not regular-bounded.

Case 2: $S \rightarrow S S$ is in $P$.

There are two subcases to consider.

(a) a $\rightarrow$ a is in P. Consider the form $F^{\prime} \rightarrow \vec{S}$ defined by the productions:

$S \rightarrow a ; S \rightarrow S S ; a \rightarrow b ; b \rightarrow b$

again $L\left(F^{\prime} \Rightarrow\right)$ is non-regular since $L\left(F^{\prime} \Rightarrow\right) \cap a^{*}$ is non-regular.

(b) a $\rightarrow$ a is not in P.

Then each production for a contains an $S$ on its right hand side, therefore construct an interpretation $F^{\prime}$ of $F$ in which

$a \Rightarrow$ "blocking" and $S \rightarrow a ; S \rightarrow S S$ are in $F^{\prime}$. Then $L\left(F^{\prime}, \Rightarrow\right)$ is non-regular.

In both cases $F$ is not regular-bounded.

Case 3: $\{a \rightarrow a\} \subseteq P$ or $\{a \rightarrow S ; S \rightarrow S\} \subseteq P$.

Case 3.1: $\{S \rightarrow$ aS; $S \rightarrow S a\} \cap P \neq \emptyset$ and $\{a \rightarrow$ aS; $a \rightarrow S a\} \cap P \neq \emptyset$. Without loss of generality assume $S \rightarrow$ as and $a \rightarrow$ as are in $P$. If $a \rightarrow a$ is in $P$ then construct an $F^{\prime} \underset{S}{\Delta} F$ with the following productions:

$S \rightarrow a A ; A \rightarrow b B ; B \rightarrow c B ; B \rightarrow d ;$

$a \rightarrow b B ; b \rightarrow b ; c \rightarrow c ; d \rightarrow e ; e \rightarrow e$

Then $L\left(F^{\prime}, \Rightarrow\right) n b c^{*} d b c^{*} d=\left\{b c^{n} d b c^{n} d: n \geq 0\right\}$, which is non-regular. If $a \rightarrow S$ and $S \rightarrow S$ are in $P$, then a similar construction suffices to show that $F$ is not regular-bounded.

Case 3.2: $\quad\{S \rightarrow$ aS; $S \rightarrow S a\} \subseteq P$.

It is easy to define an $F^{\prime} \Delta{ }_{s} F$ which generates a linear language in both cases. 
Case 3.3: $\quad\{a \rightarrow$ aS; a $\rightarrow \mathrm{Sa}\} \subseteq P$.

Again an $F^{\prime} \rightarrow F$ can be defined for which $L\left(F^{\prime} \Rightarrow\right)$ is linear.

Thus in each of the three cases considered above $F$ is not regularbounded.

Case 3.4: Either $S \rightarrow$ a $S$ or $S \rightarrow S a$ is in $P$ and $P \cap\{a \rightarrow a S ; a \rightarrow S a\}=\emptyset$. Thus $P$ consists of at most

$$
S \rightarrow a ; S \rightarrow S ; S \rightarrow a S ; a \rightarrow a ; a \rightarrow S
$$

or alternatively it has $S \rightarrow$ as replaced by $S \rightarrow S a$. Now if both $a \rightarrow a$ and $a \rightarrow S$ are in $P$ then $F$ is not regular-bounded. This follows by considering the following interpretation $F^{1} \rightarrow \vec{S}$.

$\mathrm{S} \rightarrow \mathrm{aA} ; \mathrm{A} \rightarrow \mathrm{bB} ; \mathrm{B} \rightarrow \mathrm{cB} ; \mathrm{B} \rightarrow \mathrm{d}$;

$a \rightarrow B ; b \rightarrow b ; c \rightarrow c ; d \rightarrow e ; e \rightarrow e$.

We have $L\left(F^{\prime} \Rightarrow n c^{*} d b c^{*} d=\left\{c^{n} \cdot d b c^{n} d: n \geq 0\right\}\right.$.

A similar situation occurs if both $S \rightarrow S$ and $a \rightarrow S$ are in $P$. Hence $F$ is regular-bounded only if $P \subseteq\left\{S^{\circ} \rightarrow a ; S \rightarrow S ; S \rightarrow a S ; S \rightarrow S a ; a \rightarrow a\right\}$ and $S \rightarrow$ aS and $S \rightarrow S a$ are not both in $P$.

Case 3.5: Either $a \rightarrow a S$ or $a \rightarrow S a$ is in $P$ and $P \cap\{S \rightarrow$ a $S ; S \rightarrow S a\}=\emptyset$. As in Case 3.4 if we have both $S \rightarrow S$ and $a \rightarrow S$ in $P$ or $a \rightarrow a$ in $P$ then linear languages can be generated. Consider $F^{\prime} \Delta F_{S}$ defined by the productions:

$$
\begin{aligned}
& S \rightarrow a ; B \rightarrow c ; C \rightarrow f ; D \rightarrow g ; \\
& a \rightarrow b B ; b \rightarrow c C ; c \rightarrow d D ; d \rightarrow d D ; d \rightarrow e ; e \rightarrow e D ; e \rightarrow h ; \\
& f \rightarrow f ; g \rightarrow g ; h \rightarrow k ; k \rightarrow k
\end{aligned}
$$

then $L\left(F^{\prime}, \Rightarrow n h g^{*} f h g^{*}=\left\{h^{n} f_{k g}{ }^{n}: n \geq 1\right\}\right.$.

Case 4: $P \cap\{a \rightarrow a ; a \rightarrow S\}=\emptyset$ or $P \cap\{a \rightarrow a ; S \rightarrow S\}=\emptyset$.

Case 4.1: $\quad P \cap\{a \rightarrow a ; a \rightarrow S\}=\emptyset$.

We al ready know that if either $S \rightarrow S S$ or $a \rightarrow S S$ is present

then $F$ is not regular-bounded. Therefore assume $P \cap\{a \rightarrow S S ; S \rightarrow S S\}=\emptyset$. Let $S \Rightarrow \alpha$, where $|\alpha| \geq 2$, then we claim that $|\alpha|_{a} \geq 1$ and $|\alpha|_{S} \geq 1$.

This is clearly true for $|a|=2$, since one of the productions $S \rightarrow S a$; $S \rightarrow a S ; a \rightarrow S a ; a \rightarrow$ aS must have been used in its derivation. For $|\alpha|>2$ we have $S \Rightarrow^{+} \beta \Rightarrow^{+} \alpha$ with $|\beta|=2$, that is $\beta=$ as or Sa. Now note that a derives in one step either as or sa hence by induction we can prove that $\alpha$ contains at least one a and at least one $S$. Thus $L(F, \Rightarrow)=\{a\}$ and $F$ is regular-bounded. 
Case 4.2: $\quad \mathrm{P} \notin\{a \rightarrow a ; S \rightarrow S\}=\emptyset$.

This is similar to 4.1 , namely if $P$ contains either $S \rightarrow S S$ or $a \rightarrow S S$ then $F$ is not regular-bounded and otherwise it is regularbounded and $L(F, \Rightarrow)=\{a\}$.

Using these results we can now check any supershort form by reference to the following diagram. Each box in this diagram represents a question; either implicitly by listing productions, which are a11 to be in P or explicitly by asking if a particular set is empty or nonempty. All exits are labelled with either "RB" or "not RB", where $R B$ denotes regular-bounded.

\section{enter here with $P$}

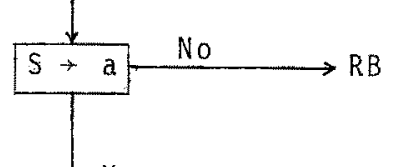

Yes

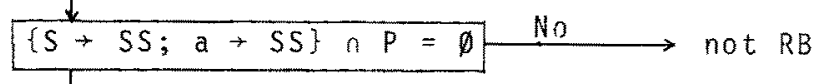

Yes
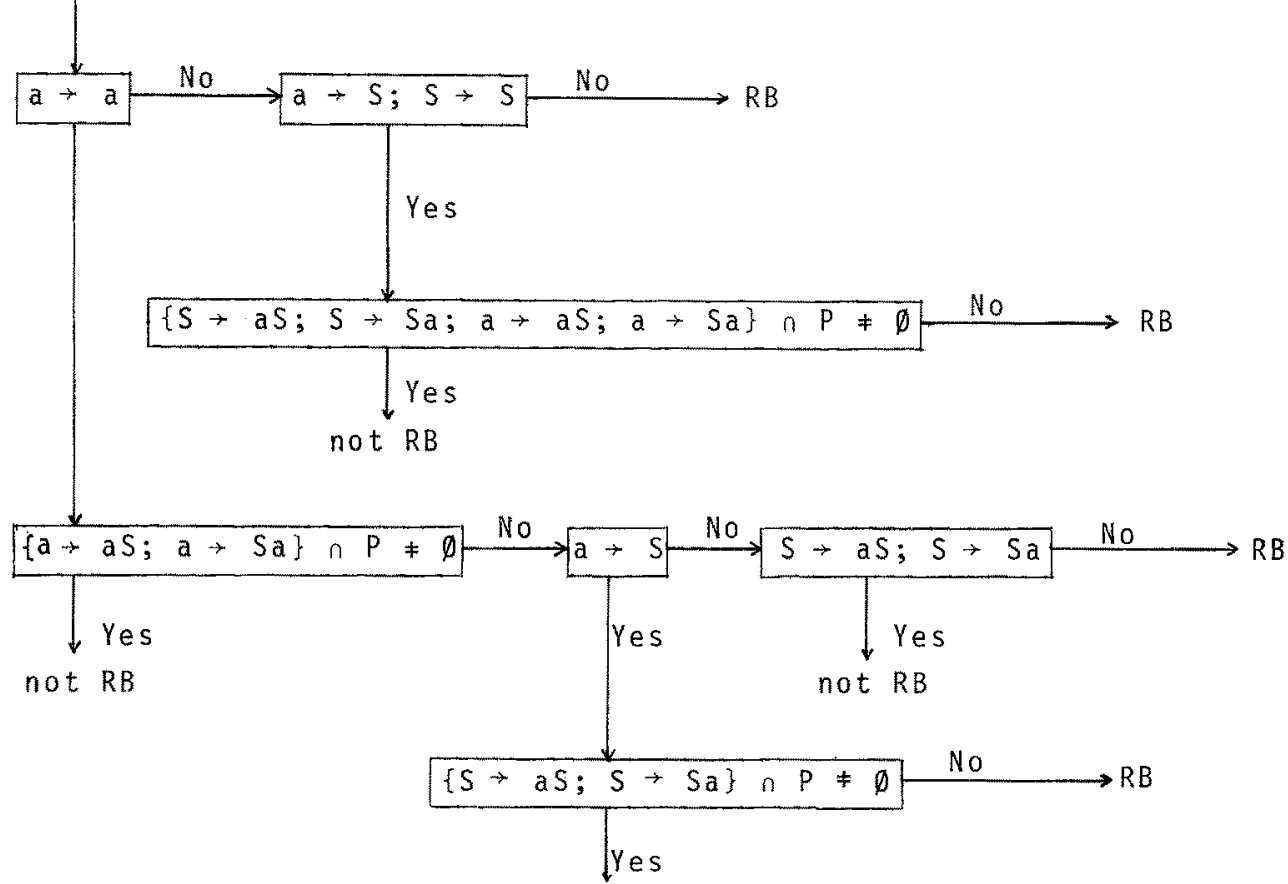

not $\mathrm{RB}$ 


\section{III.6.4 Generators}

No discussion of the generative capacity of EOL forms would be complete without some mention of the notion of a generator. In this section we take the opportunity to introduce this notion and present some preliminary results.

Intuitively, for an EOL form family $\mathcal{L}$ a language $L$ in $\mathcal{L}$ is said to be a generator for $\mathcal{X}$ if whenever $L=L(F, \Rightarrow)$ for some $E O L$ form $F, \mathcal{L}_{\mathrm{s}}(\mathrm{F}, \Rightarrow) \geq \mathcal{L}$, that is $\mathrm{F}$ is $\mathcal{L}$-sufficient. However this preliminary definition is not restrictive enough to give any significant results. For example letting $L=a^{*}$ we might expect to obtain at least the finite languages. That this is not the case is seen by considering the form:

$F: S \rightarrow a ; a \rightarrow a a ; a \rightarrow a a a$, clearly $L(F, \Rightarrow)=a *$ but $\mathcal{L}_{s}(F, \Rightarrow)$ contains no finite languages.

The restriction we consider to avoid the problem caused by terminal forcing is synchronization. We define a language $L$ to be a generator of a language family $\mathcal{L}$ if for every synchro-EOL form $F$, $L(F, \Rightarrow) L$ implies $F$ is $\mathcal{Z}$-sufficient. We say $L$ is a proper generator of $\mathcal{Z}$ if $L$ is a generator of $\mathcal{L}$ and $L$ is in $\mathcal{L}$.

We show that $\mathcal{Z}$ (FIN) has a generator but no proper generator; we exhibit a proper generator of $\mathcal{Z}$ (REG) and characterize all such generators; and we establish that $\mathcal{L}$ (LIN), $\mathcal{L}$ (CF) and $\mathcal{L}$ (EOL) have no proper generators. Whether or not $\mathcal{L}$ (LIN) or $\mathcal{L}(C F)$ have any generators is an open problem.

We first characterize the generators of $\mathcal{L}(R E G)$.

\section{Theorem 6.7}

A language $L \subseteq \Sigma^{*}$ is a generator of $\mathcal{L}(R E G)$ iff for some a in $\Sigma$ the language $L$ contains $a^{+}$.

Proof: if: Suppose $a^{+} \subseteq L$ and let $F$ be a synchro-EOL form with $L(F, \Rightarrow)=L$. Let $R \subseteq \Delta^{*}$ be an arbitrary regular language. Since $F$ is synchronized $\mathcal{L}_{\mathrm{S}}(\mathrm{F}, \Rightarrow)$ is closed under intersection with regular sets, hence there exists an $F^{\prime} \rightarrow F$ such that $L\left(F^{\prime}, \Rightarrow\right)=a^{+}$. Since EOL-gramar families are closed under dfl-substitution, there is an $F^{\prime \prime} \& F^{\prime}$ such that $L\left(F^{\prime \prime} \Rightarrow\right)=\Delta^{+}$. Finally, using regular intersection closure once more, there exists F"' $\vec{s} F$ with $L\left(F^{\prime \prime \prime} \Rightarrow\right)=R \cap L\left(F^{\prime \prime} \Rightarrow\right)=R$, and since $F^{\prime \prime \prime} \vec{s}$ (by transitivity of $\left.\underset{s}{A}\right), R$ is in $\mathcal{L}_{s}(F, \Rightarrow)$. Thus $\mathcal{L}($ REG $) \leq \mathcal{X}_{s}(F, \Rightarrow)$ 
only if: Suppose that for all a in $\Sigma, L$ does not contain $a^{+}$. Then for no $F^{\prime}$ \& $_{S} F$ does $L\left(F^{\prime}, \Rightarrow\right)=a^{+}$. Hence $\mathcal{L}(R E G) \perp \mathcal{L}_{s}(F, \Rightarrow)$.

Since $\mathcal{L}($ FIN $) \subseteq \mathcal{L}($ REG $)$, a ${ }^{+}$is also a generator of $\mathcal{L}$ (FIN). Clearly $\mathcal{L}$ (FIN) has no proper generators since by our earlier remarks if $L\left(F, \Rightarrow\right.$ is in $\mathcal{Z}(F I N), \mathcal{Z}_{\mathrm{s}}(F, \Rightarrow) \neq \mathcal{Z}(F I N)$. Thus we have:

\section{Corozzary 6.8}

$\mathcal{Z}$ (FIN) has a generator but no proper generator.

Our major result is the demonstration that neither $\mathcal{Z}$ (LIN) nor $\mathcal{L}(\mathrm{CF})$ have proper generators.

Given an $E O L$ form $F=(V, \Sigma, P, S)$, a symbol $X$ in $V$ is said to be active iff for some $\alpha$ in $V^{*}$ with $|\alpha|>1, X \Rightarrow^{+} \alpha$ in $F$. Inactive symbols are said to be looping.

We first prove the following technical lemma.

\section{Lemma 6.9}

Let $F=(V, \Sigma, P, S)$ be an EOL grammar with active alphabet $V_{a}$ and looping alphabet $V_{\ell}$.

Suppose each production is of one of the following three types:

(i) $A \rightarrow \alpha B B, A, B$ in $V_{a}, \alpha, B$ in $V_{\ell}^{*}$,

(ii) $A \rightarrow \alpha, A$ in $V_{a}$, $\alpha$ in $V_{\ell}^{*}$,

(iii) $C \rightarrow D, C, D$ in $V_{\ell}$.

Then $L(F, \Rightarrow$ is a linear language. Further, $L(F, \Rightarrow)$ can be generated by a linear grammar $G=(\bar{V}, \Sigma, \bar{P}, \bar{S})$ of the following kind:

$$
\bar{\nabla}=\{\bar{S}\} \cup \Sigma \cup\left\{A^{(k)}: 1 \leq k \leq t, A \text { in } v_{a}\right\} \text {, where the } A(k)
$$

are new symbols as is $\bar{S}$ and $t$ is an integer depending on $F$, $\bar{P}$ consists of the productions $S \rightarrow s^{(k)}$ for $1 \leq k \leq t$ and productions obtained from type ( $i$ ) and ( $i i)$ productions by replacing active symbols $A$ by $A(k)$ or by leaving them unchanged and by replacing looping symbols by terminals.

Proof: Let $v_{\ell}=\left\{x_{1}, \ldots, x_{m}\right\}$. For all $i \geq 0 \operatorname{define}$ the $\operatorname{sets} M(i)(x)=$ $\{a: X \Rightarrow a, a$ in $\Sigma\}$, for each $X$ in $V_{\ell}$, and let $M(i)$ denote the m-tuple $\left[M^{(j)}\left(x_{1}\right), \ldots, M^{(i)}\left(x_{m}\right)\right]$. Clearly, there exist integers, $p \geq 0, q \geq 1$ such that $M^{(p+i)}=M^{(p+i+j q)}$, for $0 \leq i \leq q$ and $j \geq 0$. Let $f=p+q$, let $\bar{s}$ and $A(k), l \leq k \leq f, A$ in $V_{a}$, be new nonterminals and let $\tau_{k}$ be the substitution defined on $V_{\ell}$ by $\tau_{k}(X)=M^{(k)}(X)$, for a $11 k \geq 0$. 
We now construct the linear grammar $G=(\bar{V}, \Sigma, \bar{P}, \bar{S})$ is the statement of the lemma. We need only specify the productions in $\bar{p}$. $\bar{P}$ contains:
(1) $\overline{\mathrm{S}} \rightarrow \mathrm{s}^{(k)}, 1 \leq k \leq f$,
$\left.\begin{array}{c}\text { (2) } A^{(t)} \rightarrow \tau_{t-1}(\alpha) B^{(t-1)} \tau_{t-1}(\beta), 2 \leq t \leq f, \\ A^{(p+1)} \rightarrow \tau_{f}(\alpha) B(f) \tau_{f}(\beta)\end{array}\right\}$
if $A \rightarrow \alpha B B$ is
a type (i)
production in $P$,

(3) $A^{(1)} \rightarrow \alpha B B$, if $A \rightarrow \alpha B B$ is a type (i) production in $P$ and $\alpha B B$ is in $\Sigma^{*}$,

(4) $A^{(t)} \rightarrow \tau_{t-l}(\alpha), 1 \leq t \leq f$, if $A \rightarrow \alpha$ is a type (ii) production in $P$.

Evidentiy $G$ contains only productions which fulfill the conditions of the theorem, hence it remains to prove that $L(G, \Rightarrow)=L(F, \Rightarrow)$.

\section{$L(F, \Rightarrow) \subseteq L(G, \Rightarrow)$.}

Consider an arbitrary $x$ in $\Sigma^{*}$.

We first show that $A_{0} \Rightarrow x$ in $F$, for $A_{0}$ in $V_{a}$ and $1 \leq k \leq f$ implies $A_{0}^{(k)} \Rightarrow^{\prime} x$ in $G$ with $k^{\prime} \leq k$.

Suppose we have $A_{0} \Rightarrow^{k} x$ in $F$. Then we have:

$$
\begin{aligned}
A_{0} & \Rightarrow \alpha_{1}^{(k-1)} A_{1} \beta_{1}^{(k-1)} \Rightarrow \alpha_{1}^{(k-2)} \alpha_{2}^{(k-2)} A_{2} \beta_{2}^{(k-2) \beta_{1}}(k-1) \\
& \Rightarrow{ }^{t-2} \alpha_{1}^{(k-t)} \ldots \alpha_{t}^{(k-t)} A_{t} \beta_{t}^{(k-t)} \ldots \beta_{1}^{(k-t)} \\
& \Rightarrow \alpha_{1}^{(k-t-1)} \ldots \alpha_{t}^{(k-t-1)}{ }_{\gamma}^{(k-t-1)} \beta_{t}^{(k-t-1)} \ldots \beta_{1}^{(k-t-1)} \\
& \Rightarrow{ }^{k-t-1} \quad x_{1} \ldots x_{t}{ }^{2 y}{ }_{t} \ldots y_{1}=x .
\end{aligned}
$$

Now $\alpha_{j}^{(k-i)} \Rightarrow \alpha_{j}^{(k-i-1)}$ and $\beta_{j}^{(k-i)} \Rightarrow \beta_{j}^{(k-i-1)}$, for $1 \leq j \leq t$, therefore $\alpha_{j}^{(k-i)} \Rightarrow^{k-i} x_{j}$ and $\beta_{j}^{(k-i)} \Rightarrow^{k-i} y_{j}, 1 \leq j \leq t$.

Further $A_{j-1} \rightarrow \alpha_{j}^{(k-j)} A_{j} \beta_{j}^{(k-j)}, 1 \leq j \leq t$ and $A_{t} \rightarrow \gamma^{(k-t-1)}$.

By the choice of productions in $\bar{P}$ we can construct a corresponding derivation of $x$ in $G$. Since we have

$$
A_{j-1}^{(k-j+1)} \rightarrow \tau_{k-j}\left(\alpha_{j}^{(k-j)}\right) A_{j}^{(k-j)} \tau_{k-j}\left(\beta_{j}^{(k-j)}\right) \text { in } \bar{p}, 1 \leq j \leq t,
$$

and hence in particular

$$
A_{j-1}^{(k-j+1)}+x_{j} A_{j}^{(k-j)} y_{j}, 1 \leq j \leq t .
$$
Similarly we have $A_{t}^{(k-t)} \rightarrow \tau_{k-t-1}\left(\gamma^{(k-t-1)}\right)$
$A_{t}^{(k-t)} \rightarrow z$. 
Thus we obtain

$A_{0}^{(k)} \Rightarrow x_{1} A_{1}^{(k-1)} y_{1} \Rightarrow^{t-1} x_{1} \ldots x_{t} A_{t}^{(k-t)} y_{t} \ldots y_{1} \Rightarrow x_{1} \ldots x_{t} z y_{t} \ldots y_{1}=x$

in $G$ and therefore

$A_{0}^{(k)} \Rightarrow^{t+1} x$ in $G$ with $t+1 \leq k$ as desired.

Now consider an arbitrary $x$ in $L(F, \Rightarrow)$ such that $s \Rightarrow x$ in $F$.

For $1 \leq k \leq f$ we have $S^{(k)} \Rightarrow^{k^{\prime}} x$ in $G$ with $k^{\prime} \leq k$ and we have $\bar{S} \rightarrow S^{(k)}$

in $\vec{P}$, hence

$$
\bar{S} \rightarrow x \text { in } G \text { in this case. }
$$

Therefore we now restrict our attention to the case $k>f$, that is $k=k_{1}+j q$ where $p+1 \leq k_{1} \leq f$ and $j \geq 1$. Two cases occur:

(a) $s \Rightarrow j q_{\gamma} \Rightarrow{ }^{k} l x$, where $\gamma$ consists of looping symbols only,

(b) $s \Rightarrow \Rightarrow^{j} q_{\alpha \beta} \Rightarrow{ }^{k}$ l $u z v=x$, where $A$ is an active symbol, $\alpha \Rightarrow k_{1} u ; A \Rightarrow{ }^{k} 1$ and $\alpha \Rightarrow^{k_{1}} v$.

In case (a) we have $\bar{S} \Rightarrow S^{\left(k_{1}\right)} \Rightarrow^{j q} x$ in $G$, while in case (b) we have $\bar{s} \Rightarrow S^{\left(k_{1}\right)} \Rightarrow^{j q_{U A}}\left(k_{1}\right) v$ in $G$ and by the above observation $A{ }^{\left(k_{1}\right)} \Rightarrow^{+} z$ in $G$. In both cases $x$ is in $L(G, \Rightarrow)$.

$L(G, \Rightarrow) \subseteq L(F, \Rightarrow)$

Consider an arbitrary $x$ in $L(G, \Rightarrow)$ in whose derivation a production

$$
A^{(p+1)} \rightarrow \tau_{f}(\alpha) B(f) \tau_{f}(\beta)
$$

has been applied $j \geq 0$ times. He have

$$
s \Rightarrow s^{\left(k_{1}\right)} \Rightarrow^{j q} u A^{\left(k_{1}\right)} v \Rightarrow^{k^{\prime}} \text { uzv with } k^{\prime} \leq k_{1} \text {. }
$$

By an argument analogous to that used above it can be seen that

$$
A \Rightarrow^{k} 1 \text { and } S \Rightarrow^{j q} \alpha A \beta \text { in } F
$$

with $\alpha \Rightarrow^{k} 1$ and $\beta \Rightarrow{ }^{k} 1 v$ in $F$. Hence $x$ is in $L(F, \Rightarrow)$ and the proof is complete.

The following result which is stated without proof is also needed.

\section{Proposition 6.10}

Every linear language $L \subseteq a * b *$ is the finite union of linear languages $L_{1}, \ldots, L_{r}$ where each $L_{i}$ is of the form: 


$$
\begin{aligned}
L_{i}= & \left\{\begin{array}{l}
k_{1} p_{i, 1}+\ldots+k_{m_{i}} p_{i, m_{1}}+p_{i, m_{i+1}} q_{i, m_{i+1}}+k_{m_{i}} q_{i, m_{i}}+\ldots k_{1} q_{i, j} ; \\
\quad k_{1}, \ldots k_{m_{i}} \geq 0
\end{array}\right\}, \\
& \text { for some } p_{i, 1}, \ldots, p_{i, m_{i+1}}, q_{i, 1}, \ldots, q_{i, m_{i+1}} .
\end{aligned}
$$

Theorem 6.11

(LIN) has no context-free generator.

Proof: We show that for every context-free language $L$ we can find a synchro-EOL form with $L(F, \Rightarrow)=L$ such that for sufficiently large $\ell$ the linear language $R=\left\{a^{n} b^{\ell n}: n \geq 1\right\}$ is not in $\mathcal{L}_{s}(F, \Rightarrow)$.

Suppose $L \subseteq \Sigma^{*}$ is a context-free generator of $\mathcal{L}$ (LIN).

For every pair $a, b$ in $\Sigma$, let $L_{a, b}=L n a * b *$ and let

$L_{c}=L \cap\left(\Sigma *-L_{a, b \text { in } \Sigma} a * b *\right)$.

clearly $L_{c}$ and each of $L_{a, b}$ are context-free.

Let $S_{u}$ be an additional nonterminal and let $L_{u}=L_{a, b \text { in } \Sigma} L_{a}, b$.

Now by Proposition $6.10 L_{u}=\bigcup_{i=1}^{r} L_{i}$, for some $r \geq 1$ and $L_{i}, 1 \leq i \leq r$. Thus $L_{u}$ can be generated by the linear grammar $G_{u}=\left(V_{u},\{a, b\}, P_{u}, s_{u}\right)$ $v_{u}=\{a, b\} \cup\left\{S_{u}\right\} \cup\{[i, k]: 1 \leq k \leq 4,1 \leq i \leq r\}$, where the $[i, k]$ are new nonterminals, and where $P_{u}$ consists only of productions of the following types:

(i) $s_{u} \rightarrow[i, 1], 1 \leq i \leq r$,

(ii) $[i, k] \rightarrow[i, k+1], k=1,2,3$

(iii) $[i, 1]+a^{p_{i, t}}[i, 1] b^{q_{i}, t}, 1 \leq i \leq r, 1 \leq t \leq m_{i}, p_{i, t} \neq 0 \neq q_{i, t}$,

(iv) $[i, 2] \rightarrow a^{p_{i}, t}[i, 2], 1 \leq i \leq r, 1 \leq t \leq m_{i}, p_{i, t} \neq 0, q_{i, t}=0$,

(v) $[i, 3] \rightarrow[i, 3] a^{q_{i}, t}, 1 \leq i \leq r, 1 \leq t \leq m_{i}, p_{i, t}=0, q_{i, t} \neq 0$,

(vi) $[i, 4] \rightarrow a^{p_{i, m_{i+1}}}{ }^{q_{i, m_{i+1}}}, 1 \leq i \leq r$.

Now the production $S_{u} \rightarrow[i, 1]$ determines for which $L_{i}$ a word is to be generated, while the generation of such a word is carried out in four stages. Type (ii) productions are used to transfer between stages. Type (iii) carry out the generation of both a's and b's, type (iv) the generation of a's only and type ( $v$ ) the generation of b's only. Finally termination occurs by using type (vi) productions. 

as follows:

We now convert $G_{u}$ to a synchro-EOL grammar $F_{u}=\left(\bar{V}_{u},\{a, b\}, \bar{P}_{u}, s_{u}\right)$

Let $\bar{v}_{u}=v_{u} \cup\{N, \bar{a}, \bar{b}\}$, where $\bar{a}$ and $\bar{b}$ are new nonterminals and $N$ is an additional nonterminal used for blocking purposes.

$$
\text { Let } \begin{aligned}
\bar{P}_{u}= & \{\bar{c} \rightarrow \bar{c}, \bar{c} \rightarrow c, c \rightarrow N: c \text { is in }\{a, b\}\} \\
v & \{N \rightarrow N\} \\
U & \left\{A \rightarrow \bar{\alpha}: A \rightarrow \alpha \text { is in } P_{u} \text { and } \bar{\alpha} \text { is } \alpha \text { with a } 1\right] \\
& \text { occurrences of a and } b \text { replaced by } \bar{a} \text { and } \bar{b}\} .
\end{aligned}
$$

Evidentiy $L\left(F_{u} \Rightarrow\right)=L\left(G_{u}, \Rightarrow\right)=L_{u}$.

Now let $F_{C}=\left(V_{C}, \Sigma, P_{C}, S_{C}\right)$ be a synchro-EOL grammar with $L\left(F_{c}, \Rightarrow\right)=L_{c}$ and $\left(\bar{V}_{u}-\{a, b\}\right) \cap V_{c}=D$ and let $S$ be a new nonterminal. Then $F=(V,\{a, b\}, P, S)$ with $V=V_{c} \cup \bar{V}_{u} u\{S\}$, $P=P_{c} \cup \bar{P}_{u} \cup\left\{S \rightarrow S_{c} ; S \rightarrow S_{u}\right\}$ is a synchro-EOL grammar with $L(F, \Rightarrow)=L$.

We are now in a position to derive a contradiction to the assumption that $L$ is a generator of $\mathcal{L}$ (LIN).

Choose some integer $\ell>\frac{q}{p}$ for each production $[i, 1] \rightarrow a^{p}[i, 1] b^{q}$ of type (iii) of $P_{u}$. We now show that $R=\left\{a^{n} b^{\ell n}: n \geq 1\right\}$ cannot be generated by any interpretation $F^{\prime}$ of $F$ contradicting the assumption that $L$ is a generator of $\mathcal{L}(L I N)$.

Assume $L\left(F^{\prime}, \Rightarrow\right)=R$ for some $F^{\prime} \& F$. Since each word $x$ of $R$ is of the form $a^{i} b^{j}, i, j \geq 1, a \neq b$ and all words of $L_{c}$ are of the form $a^{i} b^{j} c^{k}, i, j, k \geq 1, a \neq b \neq c$, no word of $R$ can be obtained as an interpretation of a word of $L_{c}$. Hence we may assume that $F^{\prime}$ only contains productions which are interpretations of the productions in $\overline{\mathrm{p}}_{u} \cup\left\{\mathrm{S} \rightarrow \mathrm{S}_{\mathrm{u}}\right\}$.

Note that $F^{\prime}$ satisfies the conditions of Lemma 6.9 , hence we can find a linear grammar $G^{\prime}=\left(V^{\prime}, \Sigma^{\prime}, P^{\prime}, S^{\prime}\right)$ with $L^{\prime}\left(G^{\prime}, \Rightarrow\right)=R$ where the productions of $G^{\prime}$ are of the following types:

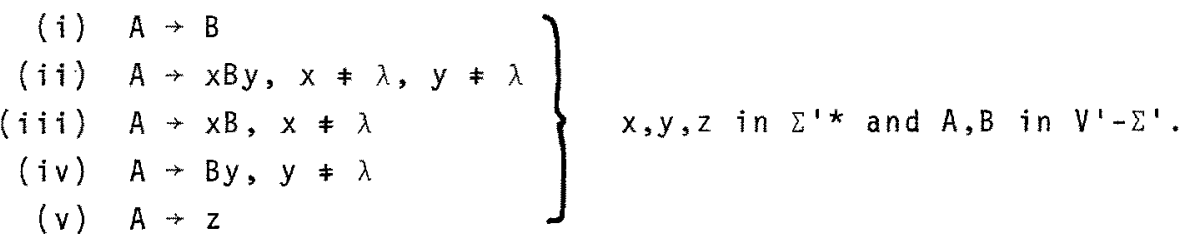

By the second part of Lemma 6.9 and the type of productions in $\bar{p}_{u}$ nonterminals occurring in type ( $\left.i i\right)$ productions cannot occur in types (iii) or (iv) productions. Similarly, in any derivation in $G^{\prime}$, nonterminals appearing in types (iii) or ( $i v$ ) productions cannot occur before nonterminals of a type (ii) production.

Let $a^{n} b^{r n}$ be a sufficiently long word. Then there is a derivation

$$
S^{\prime} \Rightarrow^{\star} u A v \Rightarrow^{*} u x A y v \Rightarrow^{*} u x z y v=a^{n} b^{r n} \text { in } G^{\prime} \text {, where } x y \neq \lambda \text { and }
$$


$u x^{i} z y^{i} v$ is in $R$, for all $i \geq 0$.

Evidently $x=\lambda, y=\lambda, x y=a^{j}$ or $x y=b^{j}$ are impossible. Hence we have $A \Rightarrow{ }^{*}{ }^{k} A b^{m}$ in $G^{\prime}$ and, by the above observation, only type (ii) productions are used in this derivation. Thus $r>\frac{m}{k}$, that is, $\frac{m}{r}<k$. Since $u \times z y b=a^{n} b^{r n}$ for some $n$ consider the word $w=u x^{2} z y^{2} v=a^{n+k} b^{r n+m}$.

We have $\frac{r n+m}{n+k}=\frac{r\left(n+\frac{m}{r}\right)}{n+k}<\frac{r(n+k)}{n+k}=r$, contradicting the assumption that $w$ is in $R$.

The proof of Theorem 6.17 also shows that for all alphabets $\Sigma, \# \Sigma \geq 2, \mathcal{L}_{\Sigma}$ (LIN), the subfamily of linear languages over $\Sigma$, has no context-free generator. Moreover it also shows that $\mathcal{L}(C F)$ has no proper generator.

\section{Coroztary 6.12}

The families $\mathcal{L}$ (LIN) and $\mathcal{L}$ (CF) do not have a proper generator.

Now for a synchro-EOL form $F, \mathcal{Z}_{S}(F, \Rightarrow)$ is closed under intersection with regular sets and under dfl-substitution. Therefore we can give a further interpretation of Theorem 6.11, namely: For each alphabet $\Sigma$ with $\# \Sigma \geq 2$ there does not exist a context-free language $L_{\Sigma}$ such that every $L$ in $\mathcal{L}_{\Sigma}(C F)$ can be obtained as $L=\tau\left(L_{\Sigma} \cap R\right)$, for some dfl-substitution $T$ and regular set $R$. This is in marked contrast to the classical Chomsky-Schutzenberger result which states that there is an $L_{\Sigma}$ such that $L$ can be obtained as $h\left(L_{\Sigma} \cap R\right)$ for some homomorphism $h$ and regular set $R$.

Although it is conjectured that $\mathcal{L}$ (LIN) has no generator at all, at the time of writing no proof is forthcoming. This conjecture, if true, would immediately imply the non-existence of a generator for $\mathcal{L}(E O L)$. For the present we estabijsh this result directly.

\section{Theorem 6.13}

$\mathcal{L}_{\{a\}}(E O L)$ has no generator, and hence $\mathcal{L}$ (EOL) has no generator.

Proof: First assume $L \subseteq\left\{a_{1}, \ldots, a_{t}\right\} *$ is a generator for $\mathcal{L}(E O L)$; Then we claim that $L \cap\left\{a_{1}^{+} \ldots a_{t}^{+}\right\}=L_{1}$, say, is a generator for $\mathcal{L}_{\{a\}}(E O L)$. For assume otherwise: that $L_{T}$ is not such a generator. Then for some synchro-EOL form $F_{1}=\left(V_{1}, \Sigma_{1}, P_{1}, S_{1}\right)$ with $L\left(F_{1}, \Rightarrow\right)=L_{1}$ and some $E O L$ language $K \subseteq a^{+} \mathcal{L}_{S}^{\prime}\left(F_{1} \Rightarrow\right)$ does not contain $K$. 
Let $L_{2}=L \cap\left(\left\{a_{1}, \ldots, a_{t}\right\}^{*}-\left(a_{1}^{+} \ldots a_{t}^{+}\right)\right)$and note that

$L=L_{1} \cup L_{2}, L_{1} \cap L_{2}=\emptyset$ and both $L_{1}$ and $L_{2}$ are EOL languages. Hence $L_{2}=L\left(F_{2} \Rightarrow\right.$ for some synchro-EOL form $F_{2}=\left(V_{2}, \Sigma_{2}, P_{2}, S_{2}\right)$. Therefore $F=(V, \Sigma, P, S)$ with

$V=V_{1} \cup V_{2} \cup\{S\}, \Sigma=\Sigma_{1} \cup \Sigma_{2}, P=P_{1} \cup P_{2} \cup\left\{S \rightarrow S_{1} ; S \rightarrow S_{2}\right\}$

is a synchro-EOL form such that $L(F, \Rightarrow)=L$. (We assume

$\left(V_{1}-\Sigma_{1}\right) \cap\left(V_{2}-\Sigma_{2}\right)=\emptyset$ without any loss of generality.) Since $L$ is a generator of $\mathcal{L}(E O L)$, there exists $F^{\prime} \bigotimes_{s} F$ such that $L\left(F^{\prime} \Rightarrow\right)=k$. From the defintion of $F$ we may assume that

$F^{\prime} \Delta_{S}\left(V_{1} \cup\{S\}, \Sigma_{1}, P_{1} \cup\left\{S \rightarrow S_{1}\right\}, S\right)$, and hence there is an interpretation $F_{j} \Delta F_{1}$ with $L\left(F_{j}, \Rightarrow\right)=K$, a contradiction.

Since $\mathcal{Z}_{\{a\}}(E O L)$ has a generator if $\mathcal{Z}(E O L)$ has a generator, we now proceed by demonstrating that $\mathcal{L}_{\{a\}}(E O L)$ does not have a generator. This in turn implies $\mathcal{Z}$ (EOL) does not have a generator.

By the first part of the proof we may assume that if an EOL language $L$ is a generator of $\mathcal{Z}_{\{a\}}(E O L)$ then $L \subseteq a_{1}^{+} \ldots a_{t}^{+}$, for some a) phabet $\left\{a_{1}, \ldots, a_{t}\right\}$. Assume such a generator $L$ exists.

Let $L=b_{j}^{+} \cup \ldots u b_{r}^{+} \cup M$, where $M \subseteq c_{1}^{*} \ldots c_{s}^{*}$ with $\left\{b_{1}, \ldots, b_{r}\right\} \cap\left\{c_{1}, \ldots, c_{s}\right\}=\emptyset$ and $c_{i}^{+}$is not contained in $L, 1 \leq i \leq s$.

For $i=1, \ldots, s$ let $F_{i}=\left(V_{i},\left\{c_{i}\right\}, P_{i}, s\right)$ be synchro-EOL forms with $L\left(F_{j}, \Rightarrow\right)=\| \cap c_{i}^{+}$.

Let $G=\left(U,\left[b_{1}, \ldots, b_{r}\right\}, Q, S\right)$ be the synchro-EOL form with $U=\left\{S, N, b_{1}, \ldots, b_{r}\right\} \cup\left\{A_{j}, B_{j}: 1 \leq i \leq r\right\}$ and

$Q=\left\{S \rightarrow A_{i}: 1 \leq i \leq r\right\} \cup\left\{A_{i} \rightarrow B_{i} A_{i}, A_{i} \rightarrow B_{i}: I \leq i \leq r\right\} u$ $\left\{B_{i} \rightarrow B_{i}, B_{i} \rightarrow b_{i}: 1 \leq i \leq r\right\} \cup\left\{b_{i} \rightarrow N: 1 \leq i \leq m\right\} \cup\{N \rightarrow N\}$.

Assume that the nonterminal alphabets are mutually disjoint with the exception of $S$. Note that $L(G, \Rightarrow)=b_{j}^{+} u \ldots u b_{r}^{+}$.

Now consider the synchro-EOL form $F$ constructed as the "union" of the $F_{i}$ and $G$. Let $F=\left(V,\left\{a_{1}, \ldots, a_{t}\right\}, P, s\right)$ where $V=U u \sum_{i=1}^{r} V_{i}$ $P=Q \cup \bigcup_{j=1}^{r} P_{i} . \quad C$ early $L(F, \Rightarrow)=L$.

Choose $s+1$ prime numbers $p_{1}, \ldots, p_{s+1}$, letting $p$ be the maximum chosen prime number. Consider the $s+1$ languages

$L_{j}=\left\{a^{p_{j}^{n}}: n \geq 1\right\} \cap\left\{a^{i}: i \neq j \operatorname{modu} t a p\right\}$ for $1 \leq j \leq s+1$.

Since $L$ is a generator, we have $L_{j}=L\left(G_{j} \Rightarrow\right.$ for some

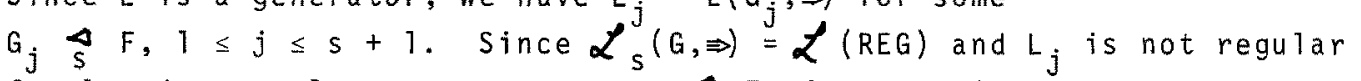
for $1 \leq j \leq s+1$, we may assume $G_{j} \underset{s}{\Delta} F_{j}$ for some $i, 1 \leq i \leq s$. Since we have $s+1$ languages $L_{1}, \ldots, L_{s+1}$ we must have $L_{j},=L\left(G_{j}, \Rightarrow\right)$ and 


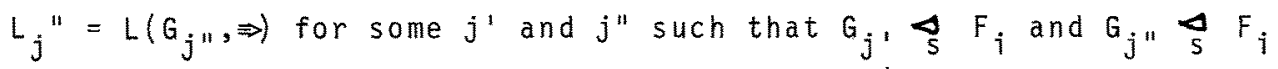
for some $i$ where $j^{\prime} \neq j^{\prime \prime}$. Since $L_{j \prime} \cup L_{j \prime}=a^{+} i t$ follows that $L\left(F_{i} \Rightarrow\right)=c_{i}^{+}$. This contradicts the assumption that $L \cap c_{i}^{+} \neq c_{j}^{+}$. 